\title{
Instrument, method, brightness, and polarization maps from the 2003 flight of BOOMERanG
}

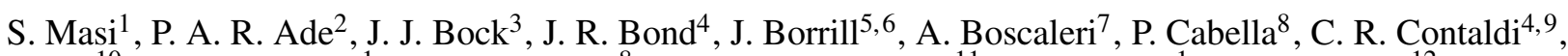
B. P. Crill ${ }^{10}$, P. de Bernardis ${ }^{1}$, G. De Gasperis ${ }^{8}$, A. de Oliveira-Costa ${ }^{11}$, G. De Troia ${ }^{1}$, G. Di Stefano ${ }^{12}$, P. Ehlers ${ }^{13}$, E. Hivon ${ }^{10}$, V. Hristov ${ }^{14}$, A. Iacoangeli ${ }^{1}$, A. H. Jaffe ${ }^{9}$, W. C. Jones ${ }^{14}$, T. S. Kisner ${ }^{15,16}$, A. E. Lange ${ }^{14}$, C. J. MacTavish ${ }^{17}$, C. Marini Bettolo ${ }^{1}$, P. Mason ${ }^{14}$, P. D. Mauskopf ${ }^{2}$, T. E. Montroy ${ }^{15}$, F. Nati ${ }^{1}$, L. Nati ${ }^{1}$, P. Natoli ${ }^{8}, 18$, C. B. Netterfield ${ }^{13,17}$, E. Pascale ${ }^{17}$, F. Piacentini ${ }^{1}$, D. Pogosyan ${ }^{4,19}$, G. Polenta $^{1}$, S. Prunet ${ }^{20}$, S. Ricciardi $^{1}$, G. Romeo ${ }^{12}$, J. E. Ruhl ${ }^{15}$, P. Santini ${ }^{1}$, M. Tegmark ${ }^{11}$, E. Torbet ${ }^{16}$, M. Veneziani ${ }^{1}$, and N. Vittorio ${ }^{8,18}$

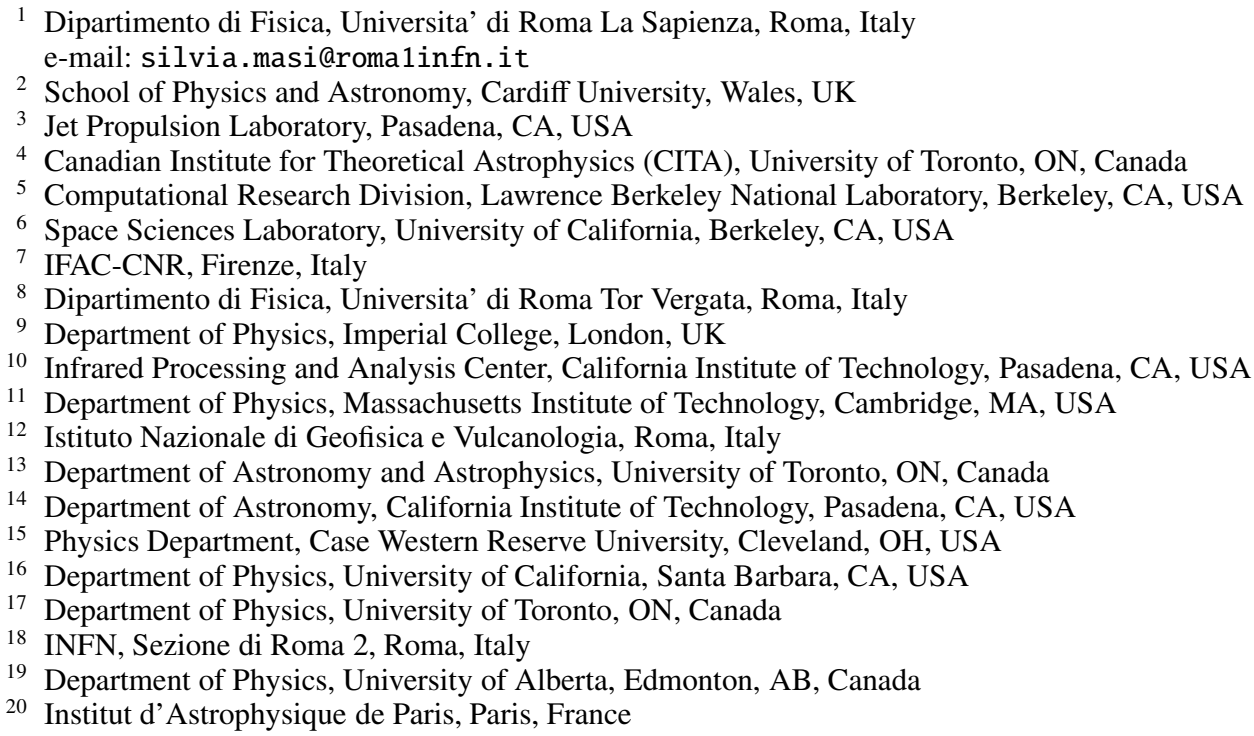

Received 23 July 2005 / Accepted 12 July 2006

\section{ABSTRACT}

\begin{abstract}
Aims. We present the BoOMERanG-03 experiment, and the maps of the Stokes parameters $I, Q, U$ of the microwave sky obtained during a 14 day balloon flight in 2003.

Methods. Using a balloon-borne mm-wave telescope with polarization sensitive bolometers, three regions of the southern sky were surveyed: a deep survey ( $\sim 90$ square degrees) and a shallow survey ( $\sim 750$ square degrees) at high Galactic latitudes (both centered at $\mathrm{RA} \simeq 5.5 h, \mathrm{Dec} \simeq-45^{\circ}$ ) and a survey of $\sim 300$ square degrees across the Galactic plane at $\mathrm{RA} \simeq 9.1 h, \mathrm{dec} \simeq-47^{\circ}$. All three surveys were carried out in three wide frequency bands centered at 145, 245 and $345 \mathrm{GHz}$, with an angular resolution of $\sim 10^{\prime}$.

Results. The $145 \mathrm{GHz}$ maps of Stokes $I$ are dominated by Cosmic Microwave Background (CMB) temperature anisotropy, which is mapped with high signal to noise ratio. The measured anisotropy pattern is consistent with the pattern measured in the same region by BOOMERanG- 98 and by WMAP. The $145 \mathrm{GHz}$ maps of Stokes $Q$ and $U$ provide a robust statistical detection of polarization of the $\mathrm{CMB}$ when subjected to a power spectrum analysis. The amplitude of the detected polarization is consistent with that of the CMB in the $\Lambda \mathrm{CDM}$ cosmological scenario. At $145 \mathrm{GHz}$, in the CMB surveys, the intensity and polarization of the astrophysical foregrounds are found to be negligible with respect to the cosmological signal. At 245 and $345 \mathrm{GHz}$ we detect ISD emission correlated to the $3000 \mathrm{GHz}$ IRAS/DIRBE maps, and give upper limits for any other non-CMB component. When compared to monitors of different interstellar components, the intensity maps of the surveyed section of the Galactic plane show that a variety of emission mechanisms is present in that region.
\end{abstract}

Key words. instrumentation: polarimeters - techniques: polarimetric - ISM: clouds - ISM: HII regions cosmic microwave background

\section{Introduction}

The Cosmic Microwave Background (CMB) is a remnant of the early Universe. Its existence is one of the pillars of the current Hot Big Bang model; its spectrum, temperature anisotropy, and polarization carry information about the fundamental properties of the Universe. The power spectrum of the temperature anisotropy of the $\mathrm{CMB},\langle T T\rangle$, is characterized by a flat plateau at scales larger than the horizon at recombination $\left(\theta \gg 1^{\circ} ; \ell \ll 200\right)$, where primordial perturbations froze early in the history of the Universe, and by a series of peaks and dips at sub-horizon scales: the signatures of acoustic oscillations of the 
primeval plasma. Measurements of angular power spectrum have been very effective in constraining cosmological parameters (see e.g. de Bernardis et al. 1994; Bond et al. 1998, 2000; Dodelson \& Knox 2000; Tegmark \& Zaldarriaga 2000a,b, Bridle et al. 2001; Douspis et al. 2001; Lange et al. 2001; Jaffe et al. 2001; Lewis \& Bridle 2004; Netterfield et al. 2002; Ruhl et al. 2003; Spergel et al. 2003; Bennet et al. 2003; Tegmark et al. 2004; Spergel et al. 2006). However, the temperature anisotropy power spectrum is degenerate in some of these parameters; independent cosmological information is required to break the degeneracy (Efsthatiou \& Bond 1999). All these studies make specific assumptions on the type of the initial conditions (adiabatic, or isocurvature) and on the shape of the power spectrum of the initial perturbations (power-law, scale-invariance, running index, etc.). When such assumptions are relaxed, the determination of the cosmological parameters becomes much more uncertain (see e.g. Bucher et al. 2002).

There is additional information encoded in the linear polarization properties of the CMB. CMB photons are last scattered at $z \sim 1100$. In Thomson scattering, any local quadrupole anisotropy in the unpolarized incoming photons creates a degree of linear polarization in the scattered photons. The main term of the local anisotropy due to density (scalar) fluctuations is dipole, while the quadrupole term is much smaller. For this reason the expected polarization is quite weak (Rees 1968; Kaiser 1983; Hu \& White 1997; Kamionkowski 1997; Zaldarriaga 2003). The polarization field can be expanded into a curl-free component (E-modes) and a curl component (B-modes). Six auto and cross power spectra can be obtained from these components: $\langle T T\rangle$, $\langle T E\rangle,\langle E E\rangle,\langle B B\rangle,\langle T B\rangle$, and $\langle E B\rangle$. Due to the parity properties of these components, standard cosmological models have $\langle T B\rangle=0$ and $\langle E B\rangle=0$. Linear scalar (density) perturbations can only produce E-modes of polarization (see e.g. Seljak 1997). In the concordance model, $\langle E E\rangle \sim 0.01\langle T T\rangle$, making $\langle E E\rangle$ a very difficult observable to measure. Tensor perturbations (gravitational waves) produce both E-modes and B-modes. If inflation happened (see e.g. Mukhanov \& Chibisov 1981; Guth \& Pi 1982; Linde 1983; Kolb \& Turner 1990), it produced a weak background of gravitational waves. The resulting level of the B-modes depends on the energy scale of inflation, but is in general very weak (see e.g. Copeland et al. 1993a,b; Turner 1993). Alternative scenarios, like the cyclic model of Steinhardt \& Turok (2002), do not produce B-modes at all (Boyle et al. 2004).

Sensitive measurements of the polarization spectra will provide a confirmation of the current scenario of acoustic oscillations in the early universe and improve the determination of cosmological parameters, in particular those related to the optical depth and reionization (see e.g. Kaplinghat et al. 2003). They will help also in detecting deviations from a simple power-law spectrum of the initial perturbations. Moreover, they will allow study of the detailed mix of adiabatic and isocurvature initial perturbations (Gordon \& Lewis 2003; Peiris et al. 2003). The detection of $\langle B B\rangle$ will probe the gravitational lensing of E-modes (Zaldarriaga \& Seljak 1998), and, if present, the inflation generated component (Leach \& Liddle 2003; Song \& Knox 2003).

Confusion by galactic foregrounds will ultimately limit the precision with which $\langle E E\rangle$ and $\langle B B\rangle$ can be measured. Not much is known about the galactic polarized background at microwave frequencies. The two mechanisms producing diffuse brightness of the interstellar medium are synchrotron radiation from relativistic electrons and thermal emission from dust.

The former is sampled at low frequencies. Patchy high latitude observations at frequencies between 0.408 and $1.411 \mathrm{GHz}$ are collected in Brouw \& Spoelstra (1976). New observations carried out with the ATCA telescope at $1.4 \mathrm{GHz}$ (Bernardi et al. 2003) and at $2.3 \mathrm{GHz}$ (Carretti et al. 2005) in the same high galactic latitude region observed by our experiment show that the polarized synchrotron emission is very weak. A naive extrapolation to $145 \mathrm{GHz}$ predicts a polarization of $0.2 \mu \mathrm{K} \mathrm{rms}$, small with respect to the $\sqrt{\langle E E\rangle} \sim 4 \mu \mathrm{K}$ expected in the concordance model.

Polarized emission of galactic dust has been detected in the $353 \mathrm{GHz}$ survey of Archeops in the Galactic Plane (Benoit et al. 2003) and at high galactic latitudes (Ponthieu et al. 2005). There, $\langle T E\rangle$ has been detected at a level of $2 \sigma$, while only an upper limit was obtained for $\langle E E\rangle$. The polarized dust emission $\sqrt{\langle E E\rangle}$ extrapolated to $145 \mathrm{GHz}$ is quite weak, less than $1 \mu \mathrm{K}$ rms. While the foreground signals are expected to be smaller than the CMB signal at $145 \mathrm{GHz}$, they should not be ignored for future, very precise measurements of CMB polarization. To do this, multiband measurements will be mandatory.

After a long pioneering phase (Caderni et al. 1978; Nanos 1979; Lubin \& Smoot 1981; Masi 1984; Partridge et al. 1988; Netterfield et al. 1995; Wollak et al. 1997), the measurement of CMB polarization is today a rapidly growing field; new interest has been sparked especially by the possibility of detecting the $\langle B B\rangle$ signature of the inflationary gravity wave background (Keating et al. 2001; Subrahmanyan et al. 2000; Hedman et al. 2002; Piccirillo et al. 2002; Delabrouille et al. 2002; Masi et al. 2002; Villa et al. 2002; Kovac et al. 2002; Johnson et al. 2003; Keating et al. 2003; Kogut et al. 2003; Farese et al. 2004; Leitch et al. 2005; Barkats et al. 2005; Readhead et al. 2004; Cortiglioni et al. 2004; Cartwright et al. 2005). To date, statistically significant detections of CMB polarization have been reported by the experiments DASI, CAPMAP, CBI and WMAP, all using coherent techniques. DASI has detected $\langle T E\rangle$ at $2.9 \sigma$ and $\langle E E\rangle$ at $6.3 \sigma$ (Leitch et al. 2005); CAPMAP (Barkats et al. 2005) has detected $\langle E E\rangle$ at $\sim 2 \sigma$; CBI (Readhead et al. 2004) has detected $\langle E E\rangle$ at $\gtrsim 7 \sigma$; WMAP has detected $\langle T E\rangle$ at many $\sigma$ (Kogut et al. 2003), and $\langle E E\rangle$ at several $\sigma$ (Page et al. 2006). The polarization power spectra measured by these experiments are all consistent with the forecast from the "concordance" model best fitting the WMAP $\langle T T\rangle$ power spectrum. Their precision, however, is not yet good enough to improve significantly the constraints on the cosmological parameters. The only exception is the $\langle T E\rangle$ measurement by WMAP at large angular scales, which provides evidence for an early, complex reionization of the universe (Kogut et al. 2003; Kaplinghat et al. 2003). The detailed structures in the $\langle E E\rangle$ spectrum are still to be confirmed, and we are very far from the sensitivity required to constrain the initial conditions or inflation.

The CMB polarization signals are so small with respect to the noise of current experiments that systematic effects are of particular concern. Consistent detection by experiments using very different techniques is important. This has been achieved only recently for CMB temperature anisotropy measurements, where the data obtained by DASI, CBI and WMAP at frequencies $\$ 100 \mathrm{GHz}$ are perfectly consistent with the bolometric maps obtained by BoOMERang, MAXIMA, ACBAR and Archeops at $150 \mathrm{GHz}$ (de Bernardis et al. 2003; Abroe et al. 2003; Kuo et al. 2002; Hamilton et al. 2003).

All detections of CMB polarization to date have been made using coherent detectors at frequencies $\$ 100 \mathrm{GHz}$. In this paper we describe a completely orthogonal experiment that has, for the first time, detected the $\mathrm{CMB}$ polarization at frequencies $\gtrsim 100 \mathrm{GHz}$. The experiment is a modification of the BOOMERanG experiment that produced the first resolved images of the CMB (de Bernardis et al. 2000) and allowed the first 
detailed extraction of cosmological parameters from the CMB (Lange et al. 2001). The modified experiment, flown in January 2003 and hereafter referred to as $\mathrm{B} 03$, is sensitive to polarization in three bands centered at 145, 245 and $345 \mathrm{GHz}$. We present here the measurement method, the instrument, and the maps of the Stokes parameters $I, Q, U$ of the CMB detected by $\mathrm{B} 03$ in the 2003 campaign.

Maps of CMB anisotropy and polarization are an important step in compressing the cosmological information into power spectra, but they are also important on their own. Maps are essential for understanding systematic effects in the measurement and the level of foreground contamination, and can be used to test the Gaussianity of the CMB fluctuations (see e.g. Polenta et al. 2002; De Troia et al. 2003; Komatsu et al. 2003; Aliaga et al. 2003; Savage et al. 2004).

Estimates of the power spectra $\langle T T\rangle,\langle T E\rangle$ and $\langle E E\rangle$ from B03 are described in three companion papers (Jones et al. 2006a; Piacentini et al. 2006; Montroy et al. 2006), and the resulting constraints on cosmological parameters in a further paper (Mac Tavish et al. 2006).

\section{The BOOMERanG-03 instrument}

\subsection{Generality}

This instrument derives directly from the BOOMERanG payload flown in 1997 (Piacentini et al. 2002) and in 1998 (Crill et al. 2003). That instrument provided the first high signal-tonoise maps of the $\mathrm{CMB}$ anisotropy with sub-horizon resolution (de Bernardis et al. 2000; Netterfield et al. 2002; Ruhl et al. 2003), and identified three peaks in the angular power spectrum of the CMB (de Bernardis et al. 2002; Ruhl et al. 2003). After the 1998/1999 flight, the instrument was recovered and modified to make it sensitive to polarization and to improve the attitude reconstruction hardware. In this section we describe the different subsystems, with focus on the new ones.

B03 is a scanning polarimeter, composed of an off-axis, $1.3 \mathrm{~m}$ diameter mm-wave telescope, a cryogenic multi-band bolometric receiver, and an attitude control system. The latter is able to control the azimuth and elevation of the telescope while the payload is floating in the stratosphere, at an altitude of $\gtrsim 30 \mathrm{~km}$, under a long duration stratospheric balloon.

We use the sky scan to modulate the signal. We map the anisotropy of the linear polarization by means of two separate bolometers, $B_{1}$ and $B_{2}$, that observe the sky through the same feed structure but are sensitive to orthogonal polarization directions. This device is called a Polarization Sensitive Bolometer (PSB, Jones et al. 2003). Each bolometer signal is processed and amplified separately.

In principle, we can then difference the two signals to obtain the Stokes parameter $Q$ of linear polarization. The $U$ parameter is measured by means of an identical PSB, containing bolometers $B_{3}$ and $B_{4}$, rotated by $\pi / 4$ in the focal plane with respect to the first one. In this minimal set of four bolometers, the principal axis of each sensor is rotated with respect to the focal plane by an angle $\alpha_{k}$. For the first PSB $\alpha_{1}=0$ and $\alpha_{2}=\pi / 2$; for the second one $\alpha_{3}=\pi / 4$ and $\alpha_{4}=3 \pi / 4$.

In practice, our sky scan strategy uses repeated scans over the same sky pixel $p$. At different times $t_{i}$ during the survey, the focal plane rotates with respect to the sky by an angle $\gamma_{i}$. Information on $Q$ and $U$ in each sky pixel thus comes from all the bolometers present in the focal plane, according to the relation

$V_{i, k}^{p}=\frac{1}{2} \mathcal{S}_{k}\left[I_{p}+Q_{p} \cos \left[2\left(\alpha_{k}+\gamma_{i}\right)\right]+U_{p} \sin \left[2\left(\alpha_{k}+\gamma_{i}\right)\right]\right]+n_{i, k} \cdot(1)$
Here $V_{i, k}^{p}$ is the signal measured by bolometer $k$ at time $t_{i} ; \mathcal{S}_{k}$ is the responsivity of bolometer $k ; I_{p}, Q_{p}, U_{p}$ are the Stokes parameters of pixel $p$ in the chosen celestial coordinates, and $n_{i, k}$ is the noise contribution to the $i$ th measurement on that pixel. This system of equations can be inverted and the Stokes parameters estimated if a sufficient number of measurements over a range of angles $\alpha_{k}+\gamma_{i}$ is taken.

Other ways to modulate the polarization involve the use of a modulating analyzer to extract the polarized component by synchronous demodulation. Rotating wire grids, half wave plates, K-mirrors, Faraday rotators, Fresnel rombs, have been used or proposed as polarization analyzers (see e.g. Keating et al. 2003; Battistelli et al. 2002; Hanany et al. 2003; Gervasi et al. 2003; Gundersen et al. 2003; Catalano et al. 2004). While all of these techniques can in principle provide a valuable means of reducing requirements on the stability of detector gains and offsets, they come at a cost in both complexity and bandwidth. Correlation polarimeters have so far been implemented only with coherent detectors (see e.g. Carretti et al. 2001; Padin et al. 2002).

The polarization measurement strategy defined by Eq. (1) is prone to leakage of the unpolarized component $I$ into the polarized ones $Q$ and $U$, if the responsivities $\mathcal{S}_{k}$ are not known exactly. Similarly, errors in the principal axes angles $\alpha_{k}$ mix $Q$ and $U$ into each other. The polarimetric calibration consists of measuring all $\mathcal{S}_{k}$ and $\alpha_{k}$. The precision required to obtain the common-mode rejection needed in our case can be estimated as follows.

For simplicity we choose the reference frame to have $\gamma=0$, and we consider the pair of detectors with $\alpha_{1}=0, \alpha_{2}=\pi / 2$. In this case we can't recover all the parameters but only $I$ and $Q$. Equation (1) becomes

$$
\begin{aligned}
& V_{1}=\frac{\mathcal{S}_{1}}{2}[I+Q]=\frac{\mathcal{S}}{2}[I+Q] \\
& V_{2}=\frac{\mathcal{S}_{2}}{2}[I-Q]=\frac{\mathcal{S} \mathcal{R}}{2}[I-Q]
\end{aligned}
$$

where we have expressed the calibration constants in terms of an absolute calibration $\mathcal{S}=\mathcal{S}_{1}$ and a relative calibration $\mathcal{R}=$ $\mathcal{S}_{2} / \mathcal{S}_{1}$. The solution is

$$
\begin{aligned}
I & =\frac{1}{\mathcal{S}}\left[V_{1}+\frac{V_{2}}{\mathcal{R}}\right] \\
Q & =\frac{1}{\mathcal{S}}\left(V_{1}-\frac{V_{2}}{\mathcal{R}}\right) .
\end{aligned}
$$

Uncertainties on the calibration constant $\sigma_{\mathcal{S}}$ and on the relative calibration $\sigma_{\mathcal{R}}$ are propagated in the error on $Q$ by

$\sigma_{Q}^{2}=\left|\frac{\mathrm{d} Q}{\mathrm{~d} \mathcal{S}}\right|^{2} \sigma_{\mathcal{S}}^{2}+\left|\frac{\mathrm{d} Q}{\mathrm{~d} \mathcal{R}}\right|^{2} \sigma_{\mathcal{R}}^{2}$

Using $I \simeq 2 V / \mathcal{S}$ (true if $Q \ll I$ )

$\frac{\sigma_{Q}^{2}}{Q^{2}}=\frac{\sigma_{\mathcal{S}}^{2}}{\mathcal{S}^{2}}+\left(\frac{1}{2} \frac{I}{Q}\right)^{2} \frac{\sigma_{\mathcal{R}}^{2}}{\mathcal{R}^{2}}$

For E-mode polarization we expect to have a factor $I / Q$ of the order of 20 . If we want to achieve a $\sim 10 \%$ accuracy in the determination of $Q$ and $U$, we need to have $\sigma_{\mathcal{R}} / \mathcal{R} \lesssim 2 \%$ for each detector. We show below that relative calibration constants can be measured within an error of $\$ 2 \%$ by comparing the CMB temperature anisotropy measured in different detectors (see Sect. 7.2.2). The absolute calibration $\mathcal{S}$ has to be determined with a relative error $\sigma_{\mathcal{S}} / \mathcal{S} \lesssim 5 \%$. 
From Eq. (1) we can also estimate the acceptable principal axis angle uncertainty. Again we choose the reference frame to have $\gamma=0$, and we consider the couple of detectors with $\alpha_{1}=$ $0, \alpha_{2}=\pi / 2$, with errors $\sigma_{\alpha_{1}}$ and $\sigma_{\alpha_{2}}$. To first order the measured Stokes parameter $Q_{\mathrm{m}}$ will be $Q_{\mathrm{m}}=Q+2 U \sqrt{\sigma_{\alpha_{1}}^{2}+\sigma_{\alpha_{2}}^{2}}$, i.e. $\left(Q_{\mathrm{m}}-Q\right) / U \simeq 2 \sqrt{\sigma_{\alpha_{1}}^{2}+\sigma_{\alpha_{2}}^{2}}$. From this equation we see that $\sigma_{\alpha_{i}} \lesssim 2^{\circ}$ produces an error in $Q$ (and $U$ ) $\lesssim 10 \%$. Mixing $Q$ and $U$ also mixes $\mathrm{E}$ and B-mode signals, so errors in alpha may affect the level at which one can set an upper limit on the B-mode polarization anisotropy signal.

Non-ideal polarized detectors have a residual sensitivity to the polarization component orthogonal to the principal axis (cross-polar response). As we rotate a perfect polarizing grid in front of a non ideal polarization sensitive detector, the detector response is given by a modified Malus law:

$V=S I_{0}\left(1-\xi \sin ^{2}\left(\alpha_{\text {det }}-\alpha_{\text {grid }}\right)\right)$,

where $\mathcal{S}$ is the responsivity, $I_{0}$ is the incident power and $\xi$ is the polarization efficiency. $\alpha_{\mathrm{det}}$ is the angle of the main axis of the detector with respect to the $\mathrm{x}$ axis of the reference frame, and $\alpha_{\text {grid }}$ is the position of the principal axis of the polarizing grid.

This can be rewritten in terms of the cross-polar response coefficient $\epsilon=V_{\perp} / V_{\|}$defining the response of the detector to radiation polarized orthogonally to its main axis, as a fraction of the response to radiation polarized parallel to its main axis. We have $\epsilon=1-\xi$. Using an operative definition of responsivity $\mathcal{S}^{\prime}=\mathcal{S}(1+\epsilon) / 2$ we rewrite

$V=\mathcal{S}^{\prime} I_{0}\left[1+\frac{1-\epsilon}{1+\epsilon} \cos \left(2\left(\alpha_{\text {det }}-\alpha_{\text {grid }}\right)\right)\right]$.

Taking into account cross-polar response, and dropping the ' for simplicity, Eq. (1) becomes

$V_{k}=\frac{\mathcal{S}_{k}}{2}\left[I+Q \frac{1-\epsilon_{k}}{1+\epsilon_{k}} \cos (2 \alpha)+U \frac{1-\epsilon_{k}}{1+\epsilon_{k}} \sin (2 \alpha)\right]+n_{k}$.

This system can be inverted to measure $Q, U$, and $I$ if the crosspolar response coefficients $\epsilon_{k}$ are known. In the same simplified case considered above the system becomes

$$
\begin{aligned}
& V_{1}=\mathcal{S}\left[I+\frac{1-\epsilon_{1}}{1+\epsilon_{1}} Q\right] \\
& V_{2}=\mathcal{R} \mathcal{S}\left[I-\frac{1-\epsilon_{2}}{1+\epsilon_{2}} Q\right] .
\end{aligned}
$$

Assuming that $\epsilon_{1}$ and $\epsilon_{2}$ are known, this is solved by

$$
\begin{aligned}
I & =\frac{1}{2 \mathcal{S}}\left[V_{1}\left(\frac{1-\epsilon_{2}}{1+\epsilon_{2}}\right)+\frac{V_{2}}{\mathcal{R}}\left(\frac{1-\epsilon_{1}}{1+\epsilon_{1}}\right)\right]\left(1+\epsilon_{1}+\epsilon_{2}\right) \\
Q & =\frac{1}{2 \mathcal{S}}\left(V_{1}-\frac{V_{2}}{\mathcal{R}}\right)\left(1+\epsilon_{1}+\epsilon_{2}\right)
\end{aligned}
$$

where the terms of the second order in $\epsilon$ have been neglected. Proceeding as above, we find

$$
\frac{\sigma_{Q}^{2}}{Q^{2}}=\frac{\sigma_{\mathcal{S}}^{2}}{\mathcal{S}^{2}}+\left(\frac{1}{2} \frac{I}{Q}\right)^{2} \frac{\sigma_{\mathcal{R}}^{2}}{\mathcal{R}^{2}}+\sigma_{\epsilon_{1}}^{2}+\sigma_{\epsilon_{2}}^{2}
$$

We conclude that in our case the uncertainty on the cross-polar response parameters can be $\Delta \sigma_{\epsilon} \lesssim 0.05$. All the conclusions obtained in this section have been verified by means of numerical simulations with realistic parameters (Masi et al. 2002).
Scanning the sky with AC-coupled detectors is an effective way to measure the intensity and polarization of the sky at the angular scales of interest for the CMB. This strategy has been used by BOOMERang-98 and Archeops, is in use in QuAD, and will be used by Planck-HFI and LFI.

In the case of B03, we scan the sky rotating the full payload in azimuth, so that atmospheric emission is almost constant along the scan. Working with an AC coupled amplifier, our system is insensitive to constant signals.

We alternate forward and reverse scans to map the low foreground region already observed by BOOMERanG-98, centered in the constellations Caelum and Horologium, at $\mathrm{RA} \simeq 5.5 \mathrm{~h}$, Dec $\simeq-45^{\circ}\left(b \lesssim-30^{\circ}\right)$. During Antarctic LDB flights, the average latitude of the payload is $\sim-78^{\circ}$. In one day, due to sky rotation, this procedure produces a highly cross-linked scan pattern (see Crill et al. 2003, Fig. 9), which is important for map making.

\subsection{Observation strategy optimization}

Our sky coverage is optimized to reduce the errors on the CMB power spectra given the constraints imposed by our telescope's hardware and the presence of bright celestial sources (the Sun and Galaxy). With the assumption of uniform sky coverage and uncorrelated noise from pixel to pixel and between $T, Q$ and $U$, the errors on $\langle E E\rangle$ are approximated by Zaldarriaga \& Seljak (1997):

$\sigma_{E, \ell}^{2}=\frac{2}{(2 \ell+1) f_{\text {sky }}}\left[C_{E, \ell}+\frac{4 \pi f_{\text {sky }} N_{E}^{2}}{\tau B_{\ell}^{2}}\right]^{2}$

and the errors on $\langle T E\rangle$ are given by

$$
\begin{aligned}
\sigma_{X, \ell}^{2}= & \frac{2}{(2 \ell+1) f_{\mathrm{sky}}} \\
& \times\left[C_{X, \ell}^{2}+\left(C_{T, \ell}+\frac{4 \pi f_{\mathrm{sky}} N_{T}^{2}}{\tau B_{\ell}^{2}}\right)\left(C_{E, \ell}+\frac{4 \pi f_{\mathrm{sky}} N_{E}^{2}}{\tau B_{\ell}^{2}}\right)\right]
\end{aligned}
$$

where $N_{T}$ and $N_{E}$ are the effective noise equivalent temperatures in $T$ and $E$ of the combined set of detectors; $\tau$ is the integration time spent uniformly covering the $f_{\text {sky }}$ fraction of the sky, and $B_{\ell}^{2}$ is the beam response in multipole space. For the case of $\mathrm{B} 03$, $N_{E}^{2}=2 \times N_{T}^{2}$ since there are eight linear detectors arranged in four orthogonal pairs, each with a different orientation on the sky (see Fig. 5). If we use an estimate of our detector sensitivity and flight time (both based on the previous flights of BoOMERanG), and a set of power spectra based on the best models to date, then we can calculate the uniform sky coverage needed to maximize the signal to noise ratio near the first peaks of $\langle E E\rangle$ and $\langle T E\rangle$. The signal to noise ratios for those band-powers are a fairly flat function of the sky coverage. The signal to noise ratio for $\langle E E\rangle$ peaks near $4 \pi f_{\text {sky }} \approx 70$ square degrees. For $\langle T E\rangle$, the peak is near $4 \pi f_{\text {sky }} \approx 1600$ square degrees (see Figs. 1 and 2 ).

The BOOMERang telescope has a useable elevation range of 35 to 55 degrees. The telescope is designed to scan no more than $60^{\circ}$ from the anti-sun direction. Exceeding this range could cause heating of the telescope baffles by sunlight. There are also constraints on the acceptable scan periods of the telescope. From the 1998 flight of BOOMERanG, we know that certain scan periods excite pendulations in the balloon-gondola system. The scan speed is restricted by the thermal time constants of the detectors, the mechanics of the telescope control systems, and the stability of our readout electronics. 


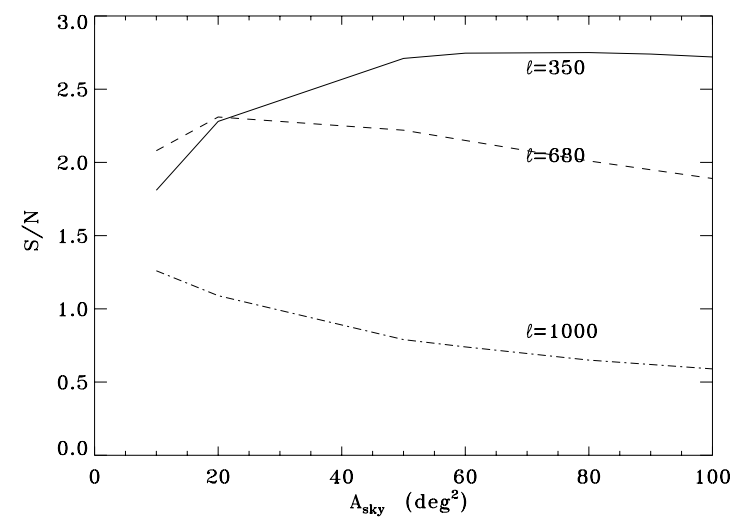

Fig. 1. The signal to noise ratio vs. sky coverage for the $\langle E E\rangle$ computed using the best estimate of $N_{E}$. The three curves refer to different $\Delta \ell=50$ bandpowers, and are labelled using the center multipole. These multipoles correspond to peaks in the "concordance" $\langle E E\rangle$ power spectrum.

Given these constraints, we created a scan strategy that came as close as possible to producing uniform coverage over both a "deep" region (for sensitivity to $\langle E E\rangle$ ) and a larger "shallow" region (for sensitivity to $\langle T E\rangle$ ). Because each change in pointing elevation perturbs the telescope, we decided to adjust the elevation no more than once per hour. With this restriction, we found that the smallest reasonable size that we could achieve for the "deep" region was $\approx 100$ square degrees. The size of our "shallow" region ( $\approx 800$ square degrees) was bounded on one side by the galaxy and on the other side by the distance from the anti-sun direction.

When determining the details of the scan strategy, we simulated the scanning of the telescope based on the same "schedule file" used to actually control the telescope during the flight. These simulations produced a coverage map for a given schedule file. Since this coverage was non-uniform, we approximated the spectral errors by the sum of the error contributions from each pixel

$$
\begin{aligned}
\sigma_{E, \ell}^{2}= & \sum_{p} \frac{2}{(2 \ell+1) f_{p}}\left[C_{E, \ell}+\frac{4 \pi f_{p} n_{p, E}^{2}}{\tau}\right]^{2} \\
\sigma_{X, \ell}^{2}= & \sum_{p} \frac{2}{(2 \ell+1) f_{p}} \\
& \times\left[C_{X, \ell}^{2}+\left(C_{T, \ell}+\frac{4 \pi f_{p} n_{p, T}^{2}}{\tau}\right)\left(C_{E, \ell}+\frac{4 \pi f_{p} n_{p, E}^{2}}{\tau}\right)\right]
\end{aligned}
$$

where $f_{p}$ is the sky fraction of a typical pixel and $n_{p, T}$ and $n_{p, E}$ are the noise in a given pixel computed from the NET of the detectors and the integration time on the pixel. We decided to spend the first four days of the flight scanning over the "shallow" region, and to spend the remainder of the flight on the "deep" region. After confirming that the scanning schedule produced the desired sky coverage (and signal to noise), we adjusted the scan speed so that it did not coincide with any pendulation modes discovered in B98. Because of these adjustments, B03 uses a range of many different azimuth scan speeds: between 0.2 and $0.6 \mathrm{deg} / \mathrm{s}$ for the deep survey, and between 0.6 and $1 \mathrm{deg} / \mathrm{s}$ in the shallow survey.

Twice a day, when the scans over the deep and shallow regions would be at nearly constant declination and therefore give

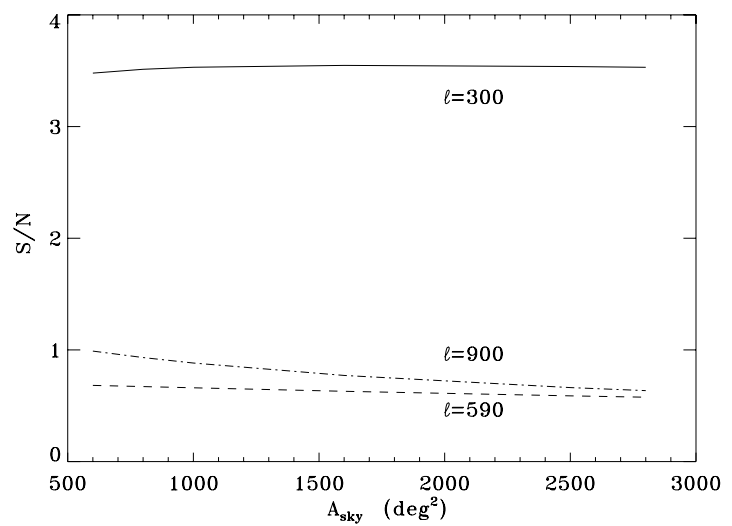

Fig. 2. The signal to noise ratio vs. sky coverage for $\langle T E\rangle$ computed using the best estimate of $N_{T}$ and $N_{E}$. The three curves refer to different $\Delta \ell=50$ bandpowers, and are labelled using the center multipole. The selected multipoles correspond to extremals in the "concordance" $\langle T E\rangle$ power spectrum.

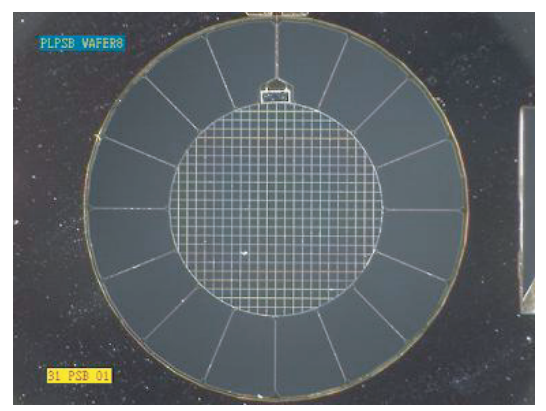

Fig. 3. Photograph of the radiation absorber of a Polarization Sensitive Bolometer used in $\mathrm{B} 03$. The $\mathrm{Si}_{3} \mathrm{~N}_{4}$ micromesh absorbing grid has a diameter of $2.6 \mathrm{~mm}$, the absorber leg spacing is $108 \mu \mathrm{m}$, and each leg is $3 \mu \mathrm{m}$ wide. Only the vertical wires are metalized. The horizontal wires are used for structural support and do not absorb radiation.

minimal cross-linking, the telescope was directed to scan a region spanning the galactic plane, including RCW38 and other galactic sources useful for calibration (see Sect. 7.4).

\subsection{Detectors}

Cryogenic bolometers are the most sensitive detectors for continuum mm-wave radiation (see e.g. Richards 1994).

The focal plane of B03 consists of eight optically active bolometric receivers, two dark bolometers, and a fixed resistor, all of which operate from a $270 \mathrm{mK}$ base temperature. The optically inactive channels are read out using identical electronics as a check against microphonics, RFI and baseplate temperature fluctuations. The focal plane is split equally between two types of receivers: four (dual-polarized) Polarization Sensitive Bolometer (PSB) pixels operating at $145 \mathrm{GHz}$, and four (singlepolarization) two-color photometers using spider-web bolometers operating at 245 and $345 \mathrm{GHz}$.

Polarization Sensitive Bolometers consist of a pair of co-located silicon nitride micromesh absorbers which couple anisotropically to linearly polarized radiation through a corrugated waveguide structure (Jones et al. 2003; Jones et al. 2006b). The system allows simultaneous background limited measurements of the Stokes $I$ and $Q$ parameters over $\gtrsim 30 \%$ bandwidths. The absorbers, separated by $\simeq 60 \mu \mathrm{m}$, are electrically and thermally isolated from one another. The devices used in $\mathrm{B} 03$ are shown in Fig. 3. 
The common thermal and radiative environment resulting from the physical proximity of the two detectors provides gain stability and rejection of differential temperature fluctuations which are limited only by differences in the properties of the NTD Ge thermistors and the electrical leads, which determine the thermal conductance to the bath. Both linear polarizations propagate through a shared waveguide structure and set of optical filters and couple to the telescope through a cryogenic corrugated feed, ensuring an identical electromagnetic bandpass and highly symmetric beams. PSBs are fabricated using the same proven photolithographic techniques used to make spider-web bolometers (Yun et al. 2003), and enjoy the same benefits of reduced heat capacity, low cross section to cosmic rays, and reduced susceptibility to microphonic response relative to monolithic bolometers.

The two-color photometer is an evolutionary development of the photometers originally designed for MAX (Fischer et al. 1992), and used subsequently by the SuZIE (Holzapfel et al. 1997), the FIRP instrument on the IRTS (Lange et al. 1994), and the Boomerang98 (Piacentini et al. 2002; Crill et al. 2003) CMB experiments. The B03 photometer has been optimized for only two frequencies, and has been made polarization sensitive by the fitting of a polarizing grid to the feed aperture. The detectors are all similar, if not identical, to the detectors flown on BOOMERanG98. The B03 feed design, consisting of a multi-mode back-to-back profiled corrugated horn, is significantly advanced relative to earlier versions of the photometer. This system achieved high efficiencies and symmetric beam patterns over the full $200-420 \mathrm{GHz}$ bandwidth.

The radiation is coupled from the photometer feed through a $420 \mathrm{GHz}$ metal mesh low-pass filter into the $12.7 \mathrm{~mm}$ diameter photometer body. A dichroic filter, tilted by $22.5^{\circ}$ with respect to the optical axis wavefront, directs radiation at frequencies above $295 \mathrm{GHz}$ to the $345 \mathrm{GHz}$ detector module while passing the lower frequencies to the $245 \mathrm{GHz}$ detector module. The detector modules are thermally isolated from the photometer body, which is held near $2 \mathrm{~K}$, by a $\sim 5$-mm gap. The photometer subKelvin feeds are smooth walled with an exit aperture matched to the geometric area of the absorber. Corrugated feeds are not necessary, as the polarization discrimination and beam forming is determined by the 2-Kelvin feed antenna.

The two configurations used in the focal plane are presented in Fig. 4. The performance of the receivers as integrated in the BOOMERang focal plane is reported in Sect. 3.

\subsection{Optical filtering}

Optical filtering is of critical importance to a bolometric receiver; in addition to defining the optical pass-band, care must be taken to ensure that the detector is shielded from out-of-band radiation originating from within the cryostat. These filters also play a significant role in the determination of the end-to-end optical efficiency of the system. Finally, the optical filtering must reduce the radiative loading on the various stages of the cryogenic system to acceptable levels.

The window of the B03 cryostat consists of a $50-\mu \mathrm{m}$ polypropilene film stretched on an elliptical aluminum frame. The window has excellent $(\gtrsim 95 \%)$ transmission at all three wavelengths but is exceedingly fragile, requiring replacement after each cycle of the cryostat.

Most of the filters used in the B03 optical system consist of layers of patterned meshes deposited on polypropylene substrates (Lee et al. 1996), with the gaps between layers filled by polypropylene as well. The dichroic beam-splitter used in

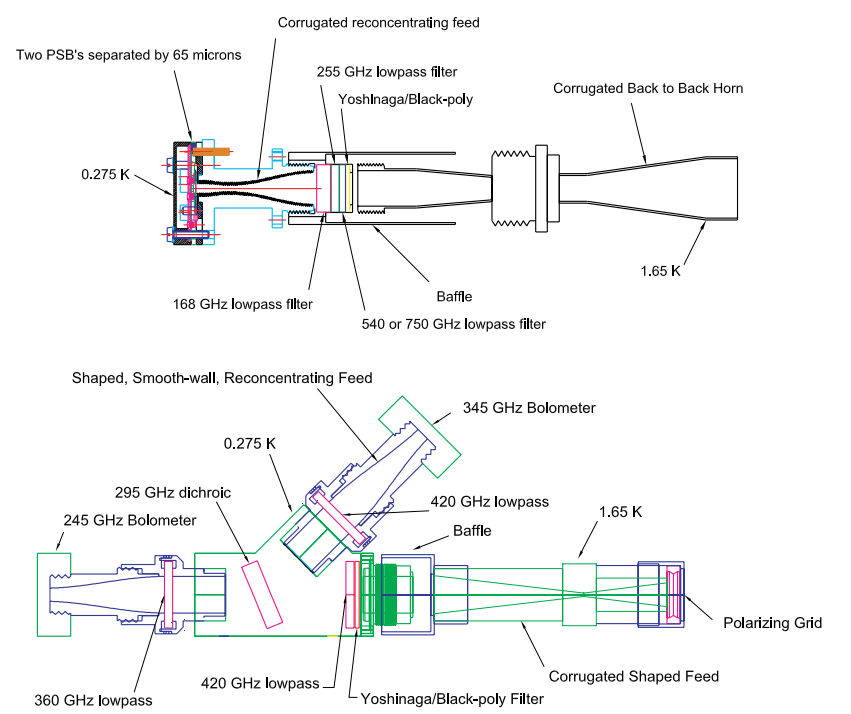

Fig. 4. Schematic of the 2-color photometers (bottom) and of the PSB polarimeters (top) used in $\mathrm{B} 03$.

the photometer body is the sole exception, being an air-gap the inductive layers are deposited on a thin Mylar substrate and stretched on an aluminum frame. The polarizing grids used on the photometer and in laboratory testing, and the neutral-density filter, are made in a similar fashion. Instead of inductive or capacitive grids, a linear pattern is used for the polarizer, while a uniform reflective coating is used for the NDF.

The layers of the filter are hot-pressed to form a single selfsupporting filter. Some of the thicker hot-pressed filters are antireflection coated with a tuned layer of PTFE (Teflon). PTFE has high infrared emissivity, which initially resulted in excessive heating of the B03 filters and a large thermal load on the $\mathrm{LN}_{2}$ and $\mathrm{L}^{4} \mathrm{He}$ stages. In addition, the heating of the filters led to a significant increase in background loading of the detectors resulting from in-band thermal emission. To ameliorate this problem, large-format, composite IR blockers were fitted in front of the $77 \mathrm{~K}$ and $2 \mathrm{~K}$ filters. These filters have high $(\gtrsim 95 \%)$ in-band transmission and reflect radiation at wavelengths shortward of $\sim 100 \mu \mathrm{m}$.

\subsection{The focal plane}

To measure polarization, we combine information from spatially separated pixels, as shown in Eq. (1). The focal plane layout (Fig. 5) is designed to minimize spatial separation between pixels, given the constraints of the existing BoOMERanG optics and the size of the feed-horns. This allows for maximal overlap of maps made by spatially separate pixels. The wide focal plane of the B03 telescope is thus populated by 8 pixels with independent corrugated feed horn systems. Each pixel contains two detectors. The four pairs of $145 \mathrm{GHz}$ PSBs in the lower row of detectors provide the best sensitivity for CMB temperature and polarization anisotropy. We have introduced some level of redundancy by using four independent PSB pairs, covering with their principal axes the range $\alpha=[0, \pi]$ in $\pi / 8$ steps. The four pixels in the upper row are 2-color photometers operating at 245 and $345 \mathrm{GHz}$. These are included to provide a lever arm for discriminating $\mathrm{CMB}$ from dusty foregrounds. Table 2 summarizes the properties of the $\mathrm{B} 03$ receiver. 
Table 1. The optical filtering scheme employed by B03. In order to take advantage of the low backgrounds available at float altitudes, much care must be taken to reduce the background originating from within the cryostat. While the metal mesh filters, which consist of bonded layers of polyethylene, exhibit in-band emissivities at the percent level, the PTFE antireflection coating is several times more emissive. It is crucial that these filters remain well heat-sunk and protected from infrared emission from the warmer stages.

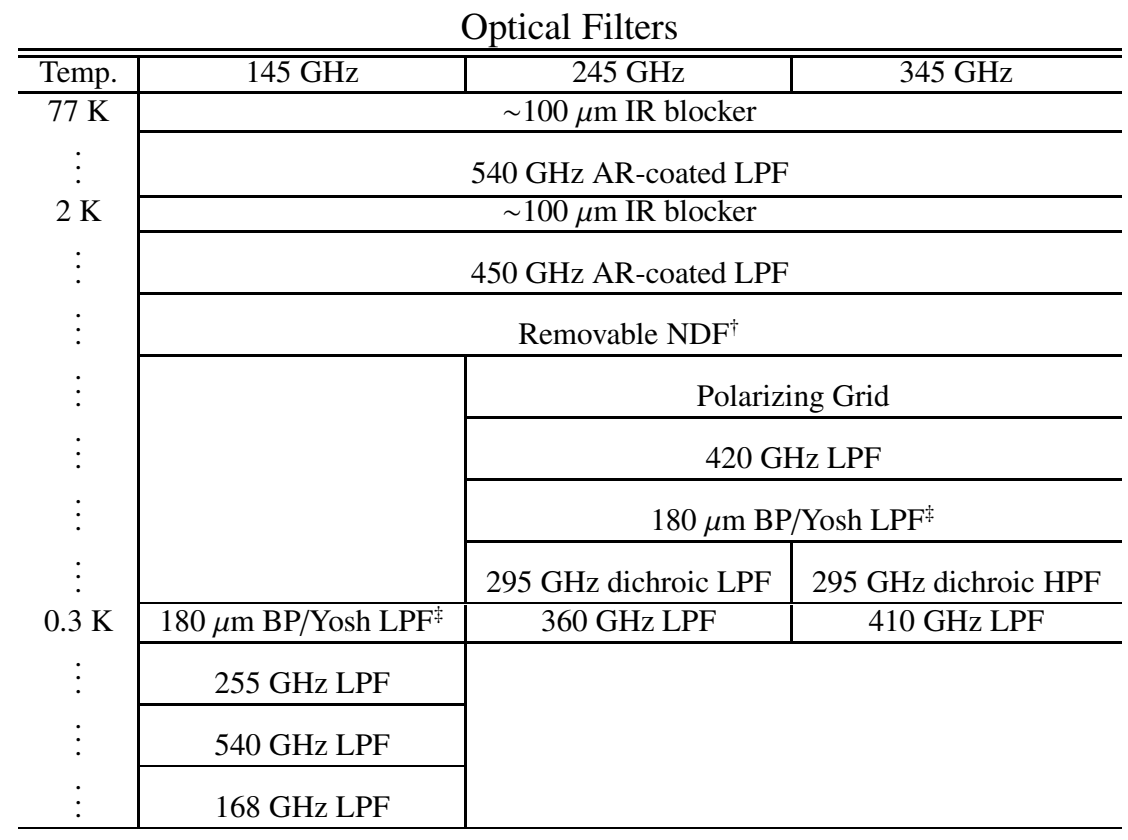

The neutral density filter can be mechanically rotated in and out of the beam while the system is cooled down.

¥ A black-polyethilene (BP) Yoshinaga filter is an absorptive filter consisting of a thallium salt deposited on a black polyethylene substrate, the thickness of which is tuned to minimize reflections (Yamada et al. 1962).

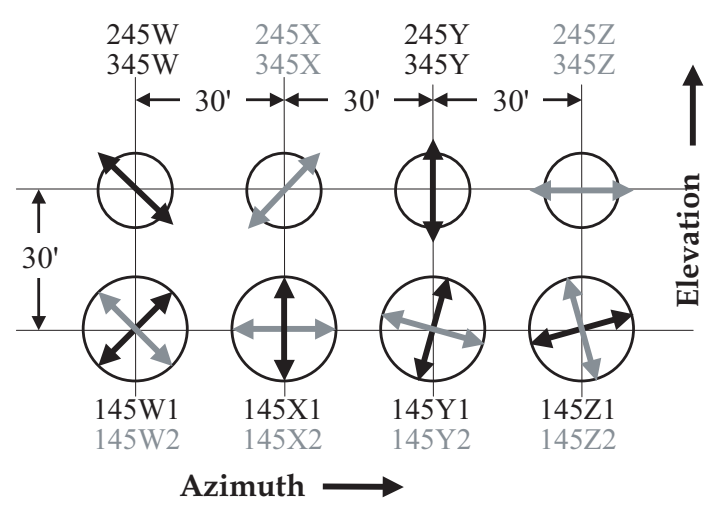

Fig. 5. Focal Plane Schematic. 2-color photometers with band centers at $245 \mathrm{GHz}$ and $345 \mathrm{GHz}$ populate the upper row. Each photometer is only sensitive to one polarization. The lower row has 4 pairs of PSB's. The elements in a PSB pair are sensitive to orthogonal polarizations. The circles representing the pixels show relative beams sizes: $\sim 7^{\prime}$ for both photometer channels and $9.5^{\prime}$ for the PSB's. The arrows through the circles show the orientation of the principal axis of polarization. The photometer and PSB rows are separated by $30^{\prime}$ in elevation, while the pixels in a row are separated by $30^{\prime}$ in cross-elevation. The labels of the two bolometers used for each pixel are also reported.

\subsection{Telescope, beam, and optics polarization properties}

The BOOMERang telescope is an off-axis system, minimizing the radiative loading on the detectors. Its configuration is close to the Dragone condition (Dragone 1974, 1982), which nulls the cross-polar response in the center of the focal plane. However, the need to accommodate a large number of detectors in the focal plane drove our optimization towards a wide corrected focal plane rather than nulling the cross-polar response only in the
Table 2. Summary of the properties of the B03 receiver. The noise reported in the last column is for a frequency of $1 \mathrm{~Hz}$ and is the average noise of all the detectors at that frequency.

\begin{tabular}{ccccc}
\hline \hline Freq. & Bandwidth & \#detectors & Beam $F W H M$ & NET $_{\mathrm{CMB}}$ \\
\hline $145 \mathrm{GHz}$ & $45 \mathrm{GHz}$ & 8 & $9.95^{\prime}$ & $170 \mu \mathrm{K} \sqrt{s}$ \\
$245 \mathrm{GHz}$ & $80 \mathrm{GHz}$ & 4 & $6.22^{\prime}$ & $320 \mu \mathrm{K} \sqrt{s}$ \\
$345 \mathrm{GHz}$ & $100 \mathrm{GHz}$ & 4 & $6.90^{\prime}$ & $450 \mu \mathrm{K} \sqrt{s}$ \\
\hline
\end{tabular}

center. We optimized the optics for diffraction limited performance at $1 \mathrm{~mm}$ over a $2^{\circ} \times 5^{\circ}$ field of view. Radiation from the sky is reflected by the parabolic primary mirror $(1.3 \mathrm{~m}$ diameter, $f=1280 \mathrm{~mm}, 45^{\circ}$ off-axis) and enters the cryostat through a thin $(50 \mu \mathrm{m})$ polypropylene window near the prime focus. Inside the cryostat, at $2 \mathrm{~K}$, the fast off-axis secondary (elliptical) and tertiary (parabolic) mirrors re-image the prime focus onto the detector focal plane. They are also configured to form an image of the primary mirror at the $10 \mathrm{~cm}$ diameter tertiary mirror, which is the Lyot-stop of the system. In the center of the tertiary mirror a $\sim 1 \mathrm{~cm}$ diameter hole hosts a thermal calibration source (callamp) which is flashed at fixed intervals during the flight (see Crill et al. 2003 for details). The size of the tertiary mirror therefore limits the illumination pattern on the primary mirror, which is under-filled by $50 \%$ in area $(85 \mathrm{~cm}$ in diameter) to improve the rejection of side-lobes. This is further improved by cold absorbing baffles surrounding the cold mirrors and rejecting stray light. Detailed parameters of the optics are described in Piacentini et al. (2002) and Crill et al. (2003).

We have studied the beams and polarization properties of this system by means of the physical optics code BMAX (Jones $2005)$. The total power beams $B(\theta, \phi)$ resulting from the system of the feed-horns and telescope are presented in Fig. 6. 


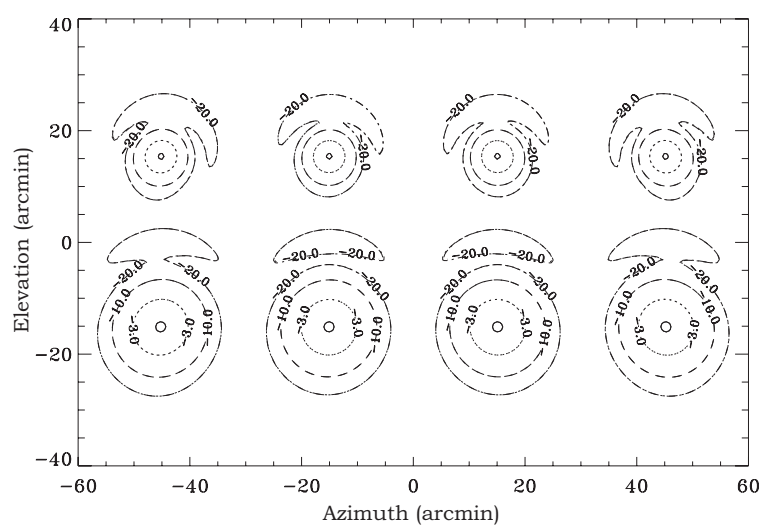

Fig. 6. Total power beams $B(\theta, \phi)$ computed for the $\mathrm{B} 03$ optical system using BMAX. The lower row is populated by the $145 \mathrm{GHz}$ PSBs, the upper row is populated by the $245 / 345 \mathrm{GHz}$ photometers. Contours are plotted for power rejection levels of $0,-3,-10,-20 \mathrm{~dB}$.

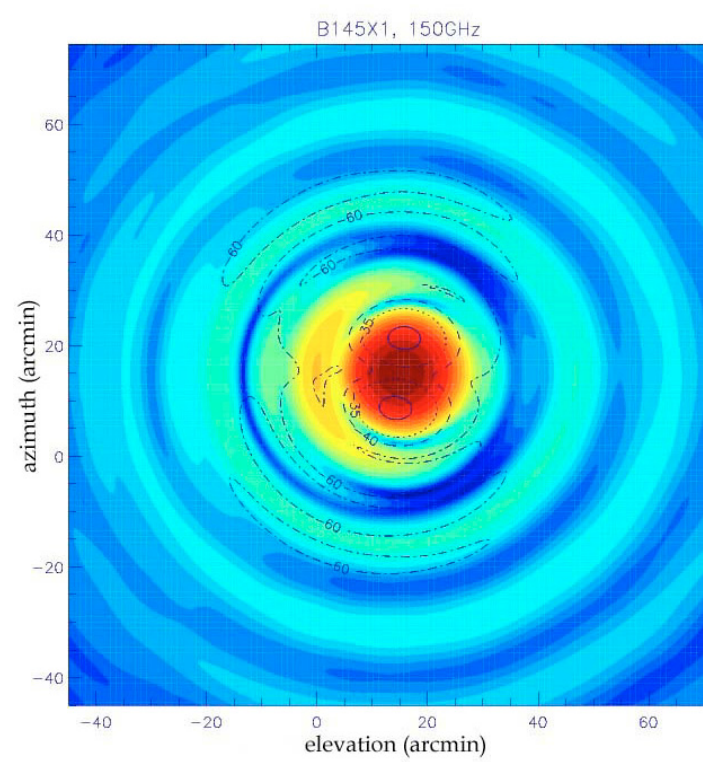

Fig. 7. Comparison of the cross-polar (contours) and co-polar (colors) beams for one of the $145 \mathrm{GHz}$ channels, as computed with the physical optics code BMAX.

In Fig. 7 we compare the cross-polar beam (contours) to the co-polar beam (colors) computed for one of the $145 \mathrm{GHz}$ channels. When integrated over $4 \pi$, the cross-polar response is $\sim$ a few $\times 10^{-3}$ of the co-polar one. We conclude that the crosspolar contribution due to the optics is negligible with respect to the one intrinsic to the detectors.

In principle, a significant ellipticity of the beam can contaminate polarization measurements. The effect depends on the details of the scan strategy. In order to estimate the acceptable level of beam ellipticity, we carried out detailed simulations of the measurement, using our scanning strategy and convolving noiseless polarized CMB maps with an elliptical beam, as in Tristam et al. (2004). The resulting simulated data have then been analyzed using our standard pipeline. We find that neglecting an ellipticity of the beam as large as $10 \%$ contaminates the estimated rms polarization by less than $0.1 \mu \mathrm{K}$ for both $Q$ and $U$. In Fig. 8 we show the effect of neglecting beam ellipticity in the estimate of polarization power spectra. As expected, the resulting contamination in the polarization power spectra is increasing with $\ell$, but is always less than a few $\%$ for a beam ellipticity
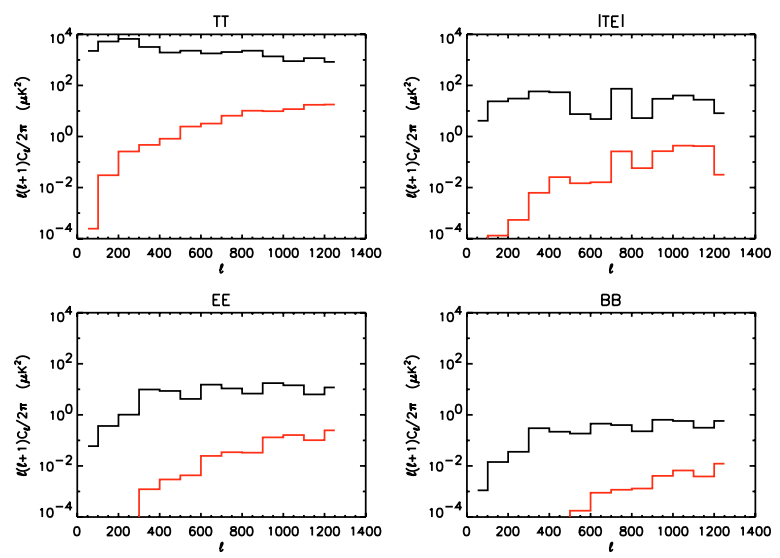

Fig. 8. Effect of ignoring a $10 \%$ beam ellipticity in the estimate of the polarization power spectra. We simulated a polarized map of the CMB sky starting from a $\Lambda-C D M C M B$ power spectrum. From the map, two noiseless simulated data sets were constructed, using the actual scanning strategy of B03. In the first set we convolved the map with a circular beam; in the second set we used an elliptical beam. Both data sets have then been analyzed using our standard pipeline, and assuming a circular beam. The top line in the plots is the power spectrum estimated from the first simulation. The lower line is the difference between the power spectra estimated from the two simulations. The relative difference is always $\lesssim$ of a few $\%$, which is much less than the statistical uncertainty we have in our measurements.

lower than $10 \%$. From the $-3 \mathrm{~dB}$ contours of Fig. 6 it is evident that the ellipticity in our case is always $\$ 10 \%$, so we expect that the effect of beam ellipticity will be negligible in our results. In Sect. 7.4 we confirm from flight data that this is indeed the case.

\subsection{Readout and signal processing}

The bolometer readout unit is the same used in the previous flights of BooMERanG (Piacentini et al. 2002; Crill et al. 2003). The B03 bolometers are AC biased with a differential sine wave at $\sim 140 \mathrm{~Hz}$. The AC voltage across the bolometer is modulated by the resistance variations induced by changes in the absorbed microwave power. A matched pair of low noise J-FETs (based on Infrared Laboratories cryogenic JFET modules) inside the cryostat reduces the signal impedance from $\sim 10 \mathrm{M} \Omega$ down to $\sim 1 \mathrm{k} \Omega$; the signal is then amplified by a differential preamp (AD624), band-pass filtered to remove noise outside the signal bandwidth, and synchronously demodulated by a phase sensitive detector (AD630). The output of the AD630 is proportional to the instantaneous resistance of the bolometer. High frequencies (above $20 \mathrm{~Hz}$, i.e. above the cutoff frequency of the bolometer) are removed by means of a 4-pole order low-pass filter. Signal (and noise) components below $5.6 \mathrm{mHz}$ are attenuated using a single pole high pass filter. A further $100 \times$ amplification is applied to fill the dynamic range of the ADC. The total amplification of the readout chain (from the bolometer to the ADC input) is 50000. This ensures that bolometer plus readout noise is more than the quantization noise of the ADC. The output signal is analog to digital converted with 16 bit resolution at the sampling frequency of $60.0 \mathrm{~Hz}$. The warm readout circuit has a gain stability of $<10 \mathrm{ppm} /{ }^{\circ} \mathrm{C}$. The readout noise is flat down to a few $\mathrm{mHz}$, and is negligible with respect to the bolometer noise with $\mathrm{NEP} \sim 1.5 \times 10^{-17} \mathrm{~W} / \sqrt{\mathrm{Hz}}$. 


\subsection{Cryogenic system}

The cryogenic system is the same flown in 1997 and 1998. The main cryostat (Masi et al. 1999) has a 65 liter tank filled with pressurized (1 atmosphere) liquid nitrogen, and a 60 liter liquid helium tank which is pumped during flight, so that $\mathrm{L}^{4} \mathrm{He}$ is superfluid. It maintains at $1.6 \mathrm{~K}$ a large (60 liters) experimental space containing the optical filters, a movable neutral density filter, and the ${ }^{3} \mathrm{He}$ refrigerator (Masi et al. 1998) cooling the bolometers and feed horn systems. The hold time of the system is around 20 days. While for the 1998 flight we used two separate entrance windows for the left and right sides of the focal plane, for the B03 flight we used a single vacuum window, roughly elliptical $(100 \mathrm{~mm} \times 65 \mathrm{~mm})$. The material is the same $50 \mu \mathrm{m}$ thick polypropylene used in 1997 and 1998.

\subsection{Attitude control system}

Azimuthal attitude control is provided by a reaction wheel with a moment of inertia approximately $0.2 \%$ of that of the complete gondola, and by a second torque motor in the pivot which couples the gondola to the flight train. The torque applied to the reaction wheel is proportional to the error in angular velocity, and the torque applied to the pivot is proportional to the rotation rate of the reaction wheel. On short time scales torque is from the reaction wheel motor, while on long time scales torque is from the pivot motor. During a $\sim 1 \mathrm{dps}$ scan, the reaction wheel has a peak rotation rate of $\sim 100 \mathrm{rpm}$. The motors are driven by a custom MOSFET bridge, controlled by a $20 \mathrm{KHz}$ PWM signal from the attitude control computer. The back-emf of the reaction wheel motor is compensated for in the motor control software, based on the reaction wheel rotation rate.

Angular velocity readout is provided by KVH ECore 2000 fibre optic rate gyroscopes, which provide an angular random walk noise of around $8^{\prime} / \sqrt{\text { hour }}$ down to $0.01 \mathrm{~Hz}$. Coarse absolute pointing is provided by a TANS-VECTOR differential GPS array (well calibrated, but with $6^{\prime}$ drifts on $\sim 20$ min time scales), and a fixed sun sensor (sub-arc-minute precision, but difficult to calibrate). The position of the inner frame relative to the outer frame is determined using a 16 bit absolute encoder. Of these sensors, only the GPS array provides a complete measure of the gondola orientation in Az, Pitch, and Roll, though with significant uncertainty.

The gondola is scanned in azimuth with a rounded saw-tooth wave form. At all times, feedback is to angular velocity from the azimuthal fibre optic rate gyroscope. During the linear part of the scan, the request velocity is constant $\left(0.3^{\circ} \mathrm{s}\right.$ typical $)$. When the end of the linear scan is reached (as determined by an absolute sensor, typically the differential GPS) the control changes from fixed angular velocity to fixed angular acceleration, until the velocity has reached the desired value in the opposite direction. The absolute sensors are only used to define the turnarounds.

The elevation is changed by moving the inner frame, which contains the cryostat, optics, and receiver readout electronics, relative to the outer frame using a geared dc motor driving a worm gear. The elevation is only changed between observing modes. During an observation, the gondola is only scanned in azimuth. No attempt is made to remove pitch motions of the outer frame by controlling the inner frame.

Two redundant, watchdog switched 80386 based computers take care of the attitude control logic, including adherence to the observation schedule file and in-flight commanding.

\subsection{Attitude reconstruction sensors}

Two pointed sensors, a pointed sun sensor and a pointed star camera, which have excellent intrinsic calibration, were added for the B03 flight.

The B03 tracking star camera (SC) consists of a video, COHU brand (4920 series), monochrome, Peltier cooled, CCD camera equipped with a Maksutov $500 \mathrm{~mm}$ focal length, f/5.6 telephoto lens. This setup yields approximately 4 arcsecond per pixel resolution and $\sim 30$ arcmin field of view. Affixed to the lens is a seven ring baffle which is covered in aluminized mylar. The interior of the baffle is painted with black water-based theatre paint to prevent light scatter from entering the optics. For the flight, a $715 \mathrm{~nm}$ high pass filter was installed and the camera gain was set to minimum in an effort to lower the risk of CCD saturation at float, in the daytime Antarctic sky.

The SC is attached to a yoke type equatorial mount. Motion control of the two axis system is provided by two Applied Motion Products high torque stepper motors. An encoder on each axis provides position feedback for controlling the motion of the mount. Motor current is controlled via pulse width modulation and a PID loop is used for the logic. Additional feedback from the azimuth gyroscope is required to facilitate star tracking while the telescope is scanning. Video images are captured with a MATROX METEOR frame grabber at a rate of $10 \mathrm{~Hz}$. The SC raw data consists of readouts from the two encoders, star pixel location in the camera field of view and star ID. Star azimuth and elevation relative to the gondola are reconstructed post-flight.

The first solar sensor (Pointed Sun Sensor, or PSS) is a motorized two axis sensor (Romeo et al. 2002) based on a four quadrant photo-diode. Unbalance on the sensor activates the motors to keep the sun spot centered on the sensor, so that the sensor accurately tracks the sun. The angles of the sensor with respect to the payload frame are measured by means of two 16 bit absolute encoders (resolution 0.33'). The second Solar sensor (Fixed Sun Sensor, or FSS) has no moving parts (Romeo et al. 2002) and is composed of two orthogonal digital meridians. In such a device a linear slit is orthogonal to a linear CCD. The sunlight entering the slit excites different pixels for different angles of incidence. The output of the sensor is the position of the center of mass of light, which can be related to the sensor-sun angle after appropriate calibration. Due to the variation of the luminosity level, an in-flight calibration is needed.

\section{Pre-flight calibration}

We extensively tested the instrument before the flight. This allowed tuning of some of the parameters for maximum performance, such as the bolometer bias frequency, preamplifier gains, and ACS sensor gains. It also allowed measurement of instrument parameters which are not expected to change from the lab to the flight, and those that cannot be measured in flight. These are the time-domain transfer function, the spectral response, the angular response, the principal axes of the polarimeters, and the cross-polar response. In the following subsections we describe the set of measurements we performed before launch.

\subsection{Transfer function}

In a scanning instrument, multipoles of the CMB fluctuations are encoded at frequencies $f$ in the time-ordered detector data:

$f \simeq \frac{\dot{\theta}}{2 \pi} \cos e \ell$ 


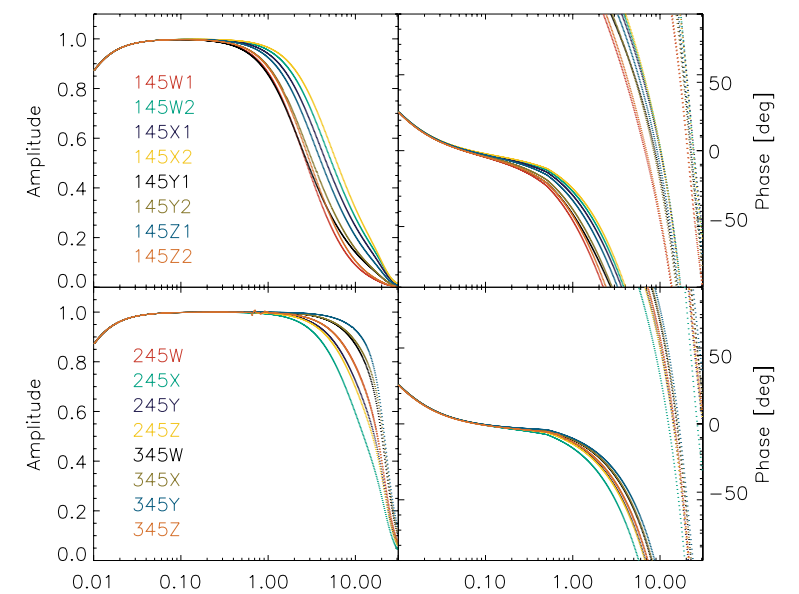

Fig. 9. Measured transfer function of all the B03 channels (color lines). 20 log-spaced frequency samples per decade have been taken. The CMB anisotropy and polarization signals of interest produce detector signals in the range $0.04 \mathrm{~Hz} \lesssim f \lesssim 3 \mathrm{~Hz}$. The low frequency response is due to the signal electronics chain. The high frequency response is affected by the individual bolometers' time constants; hence the variations at high frequencies from channel to channel.

where $\dot{\theta}$ is the sky scan speed and $e$ is the elevation of the beam. The frequency domain response of the instrument has to be known in order to deconvolve the signals obtained during the flight. In B03 we use azimuth scan speeds between 0.2 and $1 \mathrm{deg} / \mathrm{s}$ : multipoles in the range $100<\ell<1500$ produce bolometer signals at frequencies in the range $0.04 \mathrm{~Hz} \lesssim f \lesssim$ $3 \mathrm{~Hz}$. The bolometers used in B03 have thermal time constants in the $\sim 50 \mathrm{~ms}$ range. Their response is closely described by a first order low-pass filter. The readout electronics has a transfer function that matches this response and cuts low frequencies in order to remove the DC level and low frequency drifts.

The transfer functions of the full system (detectors + readout) are measured with a $77 \mathrm{~K}$ blackbody load, with a cold ( $2 \mathrm{~K}$ ) Neutral Density Filter (NDF) in the optical path. The NDF is reflective, and is composed of a thin aluminized polypropilene film. The transmission is $\sim 1.5 \%$ and fully achromatic in our wavelength range. The NDF is mounted in the $2 \mathrm{~K}$ optics box, behind the $2 \mathrm{~K}$ filter, and can be moved in and out of the beam by means of a gearmotor mechanism. The NDF has been used to simulate the in-flight loading on the bolometers, and has been removed for all other calibration measurements, since its polarization behaviour is quite complex. Looking at the $77 \mathrm{~K}$ load through the cold NDF we were able to simulate the flight background very well, as confirmed by the measurement of the inflight DC level across the detectors. The source is a modulated signal obtained chopping a small $300 \mathrm{~K}$ source against the $77 \mathrm{~K}$ blackbody background (filling about $5 \%$ of the beam solid angle). The results are reported in Fig. 9, where we plot the transfer function of the full system (bolometers plus readout electronics). We have also measured the transfer function of the readout electronics alone, and also the transfer function of the bolometers alone. The latter was obtained with the bolometers DC biased at the same current as in flight, using the NDF in the beam and observing an external liquid nitrogen load. For this measurement we have excluded the readout electronics and have used a DC amplifier with flat response up to $800 \mathrm{~Hz}$. From the comparison of the transfer functions obtained in the three ways we confirm that the high-frequency cut-off evident in Fig. 9 is largely due to the bolometers, and derive the bolometer time constants. These are between 50 and $100 \mathrm{~ms}$ for the PSBs, and between 5
Table 3. Spectral and time response calibrations: for all channels (named in Col. 1) we report in the second column the average frequency, and in the third column the optical bandwidth $\Delta v=$ $\left(\int e(v) \mathrm{d} v\right)^{2} / \int e^{2}(v) \mathrm{d} v$. Here $e(v)$ are the transmission spectra of Fig. 10. The conversion factor between Specific Brightness and CMB temperature fluctuations, as computed from the same spectra, is reported in the fourth column. Spectral normalizations and flat band optical efficiencies are measured using NDF-up load curve power differences. The spectral normalization $\eta$ (fifth column) is calculated using the spectral response from the FTS measurements. The optical efficiency $\langle\eta e(v)\rangle_{\text {band }}$ (sixth column) is calculated assuming a flat spectral response. The detectors are assumed to be single-moded. The high optical efficiency of the $345 \mathrm{GHz}$ channels is likely due to propagation of multiple modes to the detector. The time constant $\tau$ is reported in the seventh column, as measured in the laboratory with loading conditions similar to the flight ones.

\begin{tabular}{lcccccc}
\hline \hline Channel & $\langle v\rangle(\mathrm{GHz})$ & $\Delta v(\mathrm{GHz})$ & $\frac{\mathrm{MJy} / \mathrm{sr}}{K_{\mathrm{CMB}}}$ & Spec. Norm. & \multirow{2}{*}{ Opt. Eff. $\tau(\mathrm{ms})$} \\
\hline $145 \mathrm{~W} 1$ & 147.3 & 46.9 & 388 & 0.35 & 0.26 & 89 \\
$145 \mathrm{~W} 2$ & 146.7 & 45.3 & 387 & 0.35 & 0.26 & 50 \\
$145 \mathrm{X} 1$ & 146.2 & 46.1 & 386 & 0.37 & 0.28 & 58 \\
$145 \mathrm{X} 2$ & 146.6 & 45.5 & 387 & 0.33 & 0.25 & 43 \\
$145 \mathrm{Y} 1$ & 147.0 & 46.7 & 388 & 0.38 & 0.29 & 97 \\
$145 \mathrm{Y} 2$ & 146.8 & 46.6 & 387 & 0.38 & 0.31 & 82 \\
$145 \mathrm{Z} 1$ & 147.0 & 46.2 & 388 & 0.26 & 0.19 & 66 \\
$145 \mathrm{Z} 2$ & 147.1 & 47.6 & 388 & 0.21 & 0.14 & 81 \\
\hline $245 \mathrm{~W}$ & 248.0 & 94.6 & 461 & 0.38 & 0.33 & 12 \\
$245 \mathrm{X}$ & 250.7 & 87.6 & 460 & 0.36 & 0.28 & 20 \\
$245 \mathrm{Y}$ & 244.0 & 89.6 & 465 & 0.33 & 0.27 & 15 \\
$245 \mathrm{Z}$ & 247.6 & 96.0 & 461 & 0.32 & 0.27 & 16 \\
\hline $345 \mathrm{~W}$ & 340.3 & 134.7 & 321 & 0.81 & 0.70 & 7.9 \\
$345 \mathrm{X}$ & 338.9 & 137.1 & 323 & 0.89 & 0.77 & 8.0 \\
$345 \mathrm{Y}$ & 344.1 & 131.7 & 314 & 0.82 & 0.72 & 4.7 \\
$345 \mathrm{Z}$ & 337.8 & 131.3 & 326 & 0.87 & 0.68 & 12 \\
\hline
\end{tabular}

and $20 \mathrm{~ms}$ for the 245 and $345 \mathrm{GHz}$ bolometers (see Table 3). We estimate a $\lesssim 10 \%$ uncertainty in applying these data to flight conditions.

\subsection{Spectral calibration}

The optical power absorbed by the bolometer is a function of the incident optical power, the spectral response of the bolometer and the optical efficiency of entire system.

For an unpolarized beam filling source and a detector sensing a single polarization, the absorbed optical power is

$W=\frac{\eta}{2} A \Omega \int B(v) e(v) \mathrm{d} v$

where $A$ is the aperture area, $\Omega$ is the beam solid angle, $e(v)$ is the spectral response of the system (normalized to 1 in the maximum), $\eta$ is the spectral normalization (i.e. $\eta e(v)$ is the actual spectral response), and $B(v)$ is the spectrum of the input source.

The optical efficiency is then defined as the average of the function $\eta e(v)$ over the bandwidth of the detector.

The spectral response of our detectors is measured using a polarized Martin-Puplett Fourier Transform Spectrometer (FTS). Figure 10 shows the details of the spectral response $e(v)$ of the different bolometers, as measured before the flight.

To normalize the spectral response, we use load curve data taken with the bolometers viewing blackbody optical loads of $77 \mathrm{~K}$ (liquid nitrogen) and $90 \mathrm{~K}$ (liquid oxygen) (Montroy et al. 2003). For load curves taken with two different optical loadings, we can assume that the incident power (optical plus 


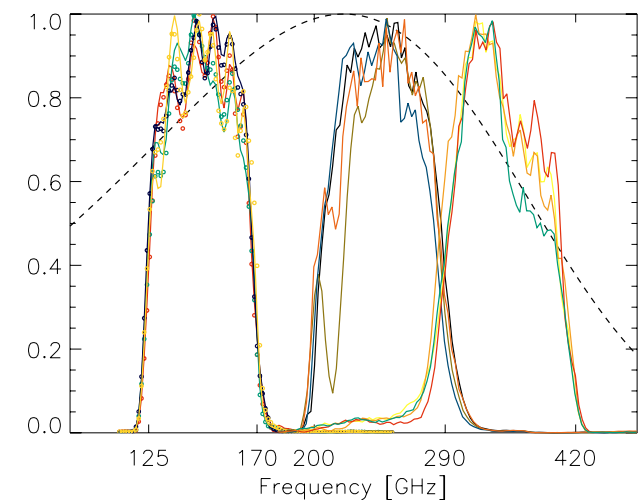

Fig. 10. Measured spectral response $e(v)$ of the 16 bolometers used in B03. The spectra have been measured before the flight by means of a thermal blackbody source and a Martin-Puplett interferometer. All the transmission measurements are normalized for unit peak transmission. The efficiencies of the different channels are reported in Table 3. The dashed line is the brightness of CMB fluctuations, normalized to the peak $(217 \mathrm{GHz})$ brightness: our three bands bracket the maximum of such a spectrum.

electrical) on the bolometer is the same when the bolometer resistance measured from the two curves is equal

$P_{\text {elec }}\left(I_{\text {bias } 1}\right)+W^{\text {load1 }}=P_{\text {elec }}\left(I_{\text {bias } 2}\right)+W^{\text {load} 2}$.

By differencing the electrical power, we can calculated the difference in optical power

$\Delta P=P_{\text {elec }}\left(I_{\text {bias } 1}\right)-P_{\text {elec }}\left(I_{\text {bias } 2}\right)=W^{\text {load2 }}-W^{\text {load1 }}$.

We calculate the spectral normalization $\eta$ from this power difference. Table 3 shows the spectral normalization and flat band optical efficiency calculated from load curve differences.

The spectral calibration of the detectors allow us to compute color corrections for all our photometric channels. In fact our system is calibrated in flight using the CMB anisotropy as a reference source (see Sect. 7.2.2). For this reason the responsivity must be corrected when observing sources with a different spectrum, like, e.g. interstellar dust emission. However, the spectral matching of PSBs is good enough that even not applying any color correction, the spurious polarization degree is $\lesssim 5 \times 10^{-3}$ for any reasonable ISD spectrum.

\subsection{Polarimetric calibration}

To characterize the polarization properties of our system, we measured the polarization efficiency, $\xi$ (or the cross-polar response $\epsilon=1-\xi$ ), and the polarization angle of each detector, $\alpha_{\text {det }}$ (see Eqs. (8) and (9)).

We made these measurements with the receiver alone and with the whole system including the telescope.

Figure 11 shows the configuration of the receiver polarization calibration. A rotating polarizing grid is placed directly beneath the cryostat. It sits above a cold load (liquid nitrogen) which is modulated by a chopper wheel. The chopper wheel is rotated at $2 \mathrm{~Hz}$ and the grid has a rotation period of approximately $10 \mathrm{~min}$. When the transmission axis of the grid is aligned with the polarization axis of the detector, the detector sees whatever is behind the grid (i.e. the chopper wheel or the cold load). When the grids are $90^{\circ}$ out of alignment the detector sees radiation reflected off the grid. The size of the source aperture is such that the modulated signal fills $\sim 5 \%$ of the receiver solid

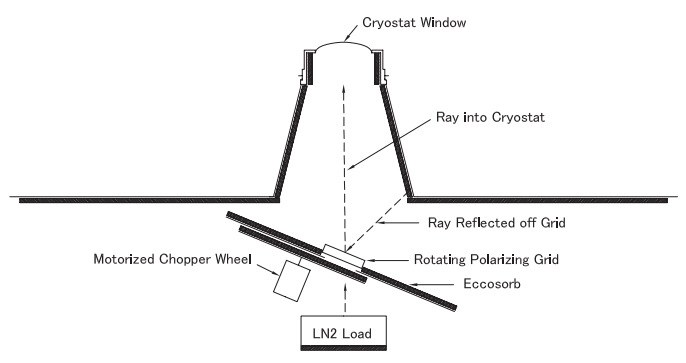

Fig. 11. The source used for the polarization calibration of the receiver. The system is tilted at $22.5^{\circ}$ so that rays reflecting off the grid go to $300 \mathrm{~K}$ eccosorb absorbers (shown as black, thick lines in the drawing). The aperture of the system is $\sim 5 \mathrm{~cm}$. The chopper wheel chops between $77 \mathrm{~K}$ and $300 \mathrm{~K}$, while a belt drive rotates the polarizing grid. The chopper wheel rotates at $2 \mathrm{~Hz}$, while the grid has a rotation period of about $10 \mathrm{~min}$.

Table 4. Cross-polarization and principal axis angle measurements for all the channels. The polarization angle and the beam filling values refer to measurements on the complete instrument obtained with the apparatus described in Fig. 12, while the on-axis measurements refer to measurements on the receiver alone, obtained with the apparatus described in Fig. 11 and also in a test cryostat testing single channels.

\begin{tabular}{lrrcc}
\hline \hline Channel & \multicolumn{2}{c}{ Polarization angle (deg) } & \multicolumn{2}{c}{ Cross-polarization $\epsilon$} \\
& nominal & measured & on-axis & beam-filling \\
\hline $145 \mathrm{~W} 1$ & 135.0 & $137.1 \pm 2.0$ & $0.080 \pm 0.006$ & $0.11 \pm 0.02$ \\
$145 \mathrm{~W} 2$ & 45.0 & $44.4 \pm 2.0$ & $0.062 \pm 0.005$ & $0.11 \pm 0.02$ \\
$145 \mathrm{X} 1$ & 180.0 & $178.4 \pm 2.0$ & $0.055 \pm 0.013$ & $0.09 \pm 0.02$ \\
$145 \mathrm{X} 2$ & 90.0 & $88.7 \pm 2.0$ & $0.070 \pm 0.008$ & $0.13 \pm 0.02$ \\
$145 \mathrm{Y} 1$ & 157.5 & $158.6 \pm 2.0$ & $0.051 \pm 0.012$ & $0.11 \pm 0.02$ \\
$145 \mathrm{Y} 2$ & 67.5 & $66.2 \pm 2.0$ & $0.060 \pm 0.006$ & $0.11 \pm 0.02$ \\
$145 \mathrm{Z} 1$ & 112.5 & $109.8 \pm 2.0$ & $0.182 \pm 0.004$ & $0.22 \pm 0.02$ \\
$145 \mathrm{Z} 2$ & 22.5 & $21.2 \pm 2.0$ & $0.088 \pm 0.008$ & $0.12 \pm 0.02$ \\
\hline $245 \mathrm{~W}$ & 135.0 & $139.2 \pm 2.0$ & $0.007 \pm 0.005$ & $0.02 \pm 0.02$ \\
$245 \mathrm{X}$ & 45.0 & $42.9 \pm 2.0$ & $0.007 \pm 0.005$ & $0.02 \pm 0.02$ \\
$245 \mathrm{Y}$ & 180.0 & $178.7 \pm 2.0$ & $0.000 \pm 0.015$ & $0.03 \pm 0.02$ \\
$245 \mathrm{Z}$ & 90.0 & $85.2 \pm 2.0$ & $0.014 \pm 0.013$ & $0.01 \pm 0.02$ \\
\hline $345 \mathrm{~W}$ & 135.0 & $139.9 \pm 2.0$ & $0.008 \pm 0.005$ & $0.01 \pm 0.02$ \\
$345 \mathrm{X}$ & 45.0 & $42.6 \pm 2.0$ & $0.008 \pm 0.001$ & $0.01 \pm 0.02$ \\
$345 \mathrm{Y}$ & 180.0 & $178.7 \pm 2.0$ & $0.004 \pm 0.014$ & $0.02 \pm 0.02$ \\
$345 \mathrm{Z}$ & 90.0 & $84.9 \pm 2.0$ & $0.019 \pm 0.013$ & $0.02 \pm 0.02$ \\
\hline
\end{tabular}

angle. The NDF was not in the beam, in order to avoid multiple reflections, which could affect the polarization response of the instrument. As a result, the background on the detectors is about hundred times higher than in flight, and the responsivity of all the detectors is many times lower. This is not a problem for these measurements since, with increased bias, there is plenty of signal anyway, and both the polarization axis position and the cross-polar response are independent of the absolute responsivity. Moreover, the modulated signal is much smaller than the background, so that the the response of the detectors, even if low, is still linear. The best fit values of the cross-polar response parameter $\epsilon$ when the source is on the optical axis are reported in Table 4. The measured cross-polar response is in principle an upper limit for the cross-polar response of our receiver, since it includes any cross-polar radiation produced by the calibration source. However, using two identical polarizing grids in series, we have verified that their transmission is $<0.1 \%$ when the principal axes form an angle of $\pi / 2$. We conclude that the contribution of the source to the measured cross-polar response is negligible. This fact is also confirmed a posteriori by the low level of total cross polarization measured in the 245 and $345 \mathrm{GHz}$ detectors. 

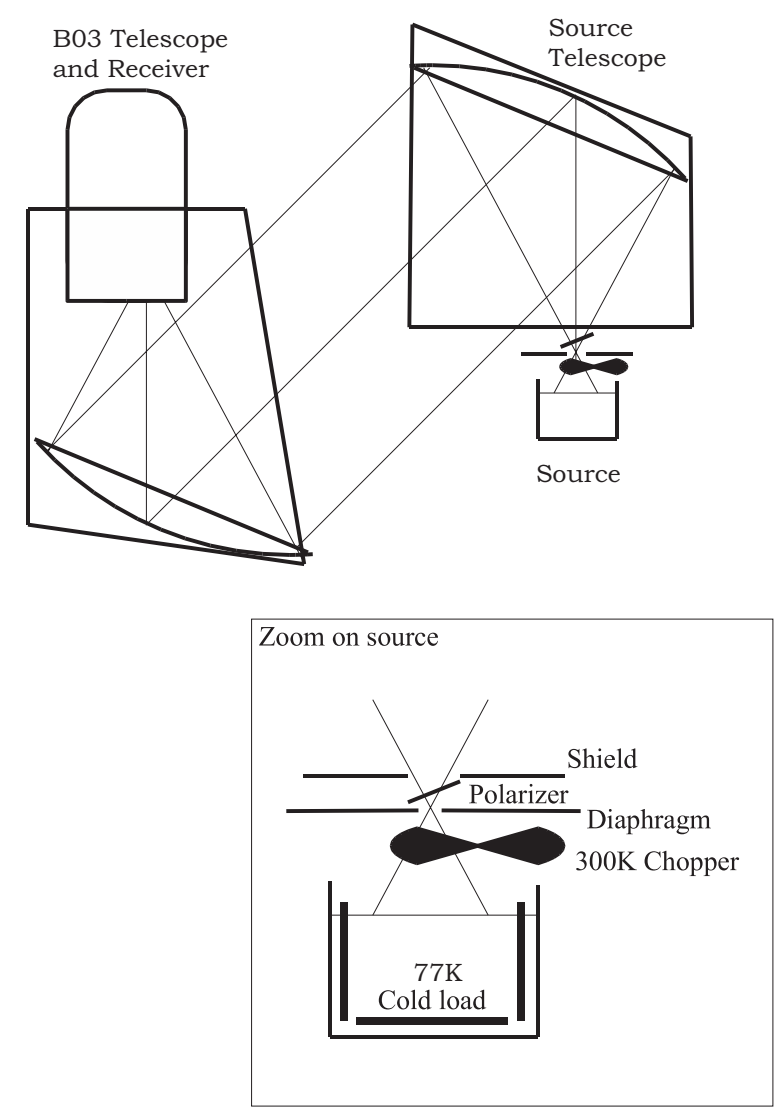

Fig. 12. The apparatus for measuring polarization angle and cross polar response of the entire telescope. The cold load is kept at $77 \mathrm{~K}$ by a liquid nitrogen bath. The source telescope is a $1.3 \mathrm{~m}$ off axis paraboloid (the spare of the B03 mirror), and is kept at a distance of $\sim 6 \mathrm{~m}$ from the B03 telescope. The source is scanned in Azimuth by rotating the B03 payload around its vertical axis. With a small diaphragm in the source, scans are repeated at different elevations to obtain a full map of the beam and of its polarization properties. With a large diaphragm, producing a beam-filling beam, we change azimuth and elevation of B03 to find the maximum signal.

For the measurement of the full system we used a fully polarized modulated plane wave filling the telescope aperture. This was produced using a thermal source in the focus of the spare BOOMERanG primary mirror. This inverted telescope system was placed in front of the B03 telescope (see Fig. 12). Analytical calculations (Dijk et al. 1974) and optical simulations show that the cross-polar response introduced by the spare primary at our frequency is $\lesssim 1 \%$. The source at the focus is made with a high efficiency wire grid (12.5 $\mu \mathrm{m}$ diameter Tungsten wires, $25 \mu \mathrm{m}$ spacing) in front of a chopper alternating a $77 \mathrm{~K}$ blackbody and a $300 \mathrm{~K}$ blackbody. The wire grid axis was tilted by $28^{\circ}$ with respect to the optical axis of the system. Again, we have verified using two identical grids in series, that their transmission is $<0.1 \%$ when the principal axes form an angle of $\pi / 2$. We conclude that also in this case the contribution of the source to the measured cross-polar response is negligible. The B03 telescope was mounted on a rotating platform with absolute encoders for angle measurements. The NDF was not in the beam, as explained above. Bolometer data and the chopper reference signals were read by the Data Acquisition System of the experiment. A software demodulator measuring amplitude and phase of the synchronous signal was used to extract the polarized source signal from the background. Data were taken for 18 positions of the wire grid between 0 and 180 degrees. After correcting for the tilt
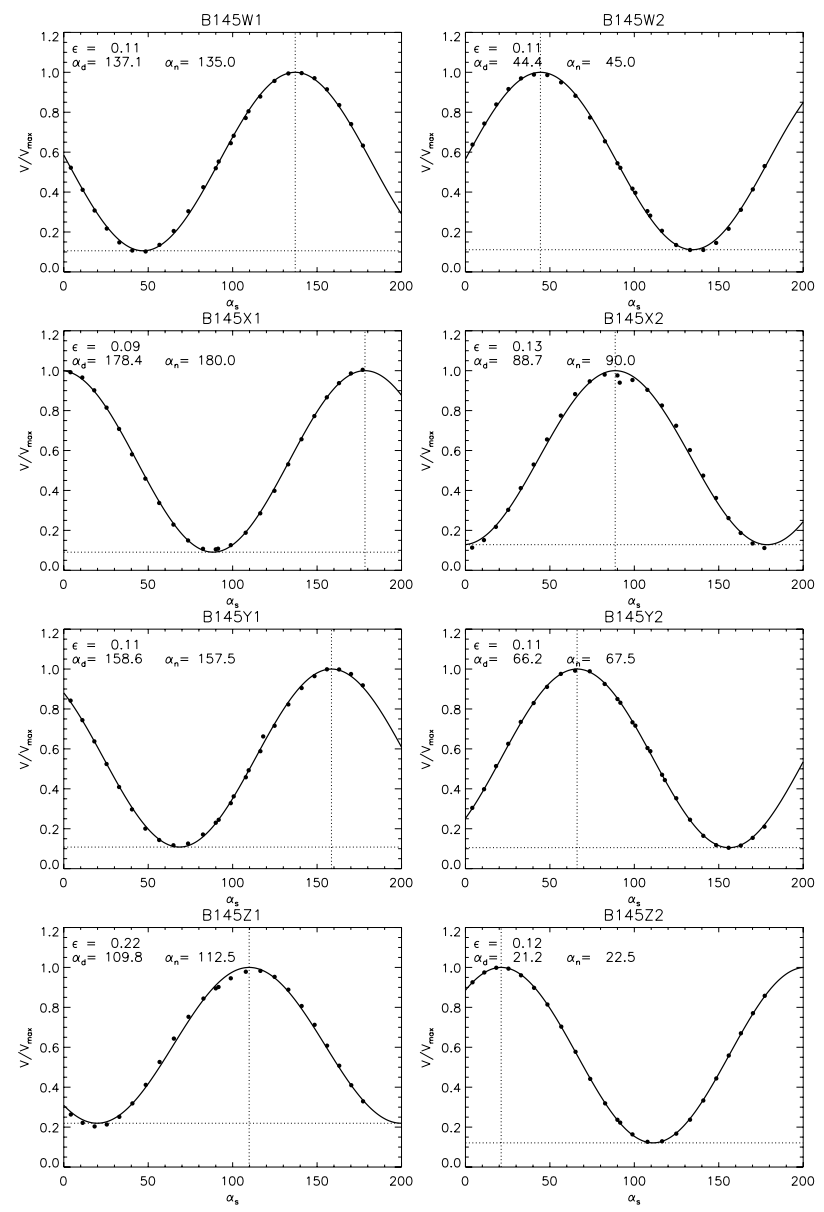

Fig. 13. Beam-integrated polarization response of the entire instrument (PSB channels).

of the grid with respect to the optical path, the data for all the bolometers and for all the incidence angles were fit with Eq. (9).

The measurement was carried out in two different configurations. In the first we used a wide aperture on the cold source, so the telescope beam was completely filled by a $\gtrsim 1^{\circ}$ source. The source-telescope relative position was then optimized for maximum signal. In this configuration we measured the integrated polarization properties of the instrument. The measured data for this configuration are shown in Fig. 13 for the PSB channels, and in Fig. 14 for the two-color photometer channels. The data shown in Figs. 13 and 14 derive from measurements where we spent a long ( $100 \mathrm{~s})$ integration time in each input polarization angle. As a result, the statistical error due to detector noise is negligible with respect to calibration errors. In the plot of Figs. 13 and 14 the statistical error is smaller than the dots representing the data. Inspection of the same plots shows that there are in some cases random deviations from the sinewave behaviour otherwise evident in the data, and that these deviations exceed the level allowed by the statistical errors. These measurements have been carried out during the pre-flight characterization of the instrument, and there was no time to trackdown the origin of the detected deviations. We believe that they derive from perturbations of the experimental setup due to the presence of other pre-flight activities. However, treating these deviations as random errors, and increasing the error bars accordingly so that the reduced $\chi^{2}$ is 1 , we find that the error in the best fit cross-polarization is still $<1 \%$, i.e. similar or lower than 

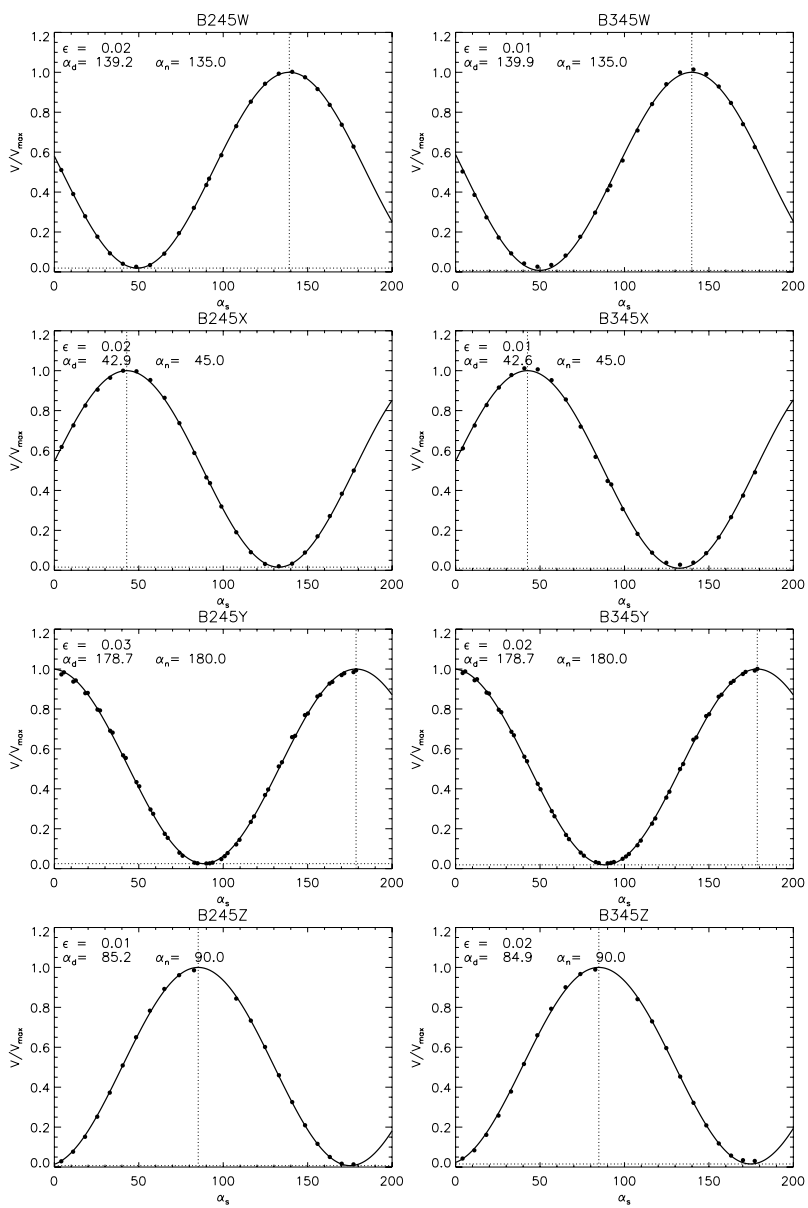

Fig. 14. Beam-integrated polarization response of the entire instrument (two-colors photometers).

the upper limit we have for the cross polarization of the source itself. For this reason we have conservatively given a $2 \%$ error for the cross-polarization measurements. This error includes the statistical noise error, the systematic error, and the source crosspolarization uncertainty.

The best fit values of the parameters $\epsilon$ and $\alpha_{\text {det }}$ are reported in Table 4. The measured cross-polar response for the PSB channels is of the order of 0.1 . It is lower for the two color photometer channels, because of the high efficiency of the wire grids. This fact confirms that the source itself cannot contribute significantly to the measured cross-polar response. In the second configuration we used a small aperture $\left(\sim 3^{\prime} F W H M\right)$, in order to investigate the cross-polar response properties as a function of the off-axis angle. In general the on-axis cross-polar response is lower than the integral values of Table 4, while off-axis is higher. This is in agreement with the physical optics model of our system (shown in Fig. 7) and with the receiver polarization calibration described above.

\subsection{Beam measurement}

We measured the beam profile $B(\theta, \phi)$ of our telescope with a thermal source tethered on a balloon in the far field of the telescope. The measurement was made in the relatively transparent atmosphere of Antarctica. A small tethered balloon lifted a microwave absorber (made out of eccosorb) into the telescope beam, at a distance of about $1.5 \mathrm{~km}$ from the payload. We used two sources to measure the beam, a sphere (with $45 \mathrm{~cm}$ diameter)

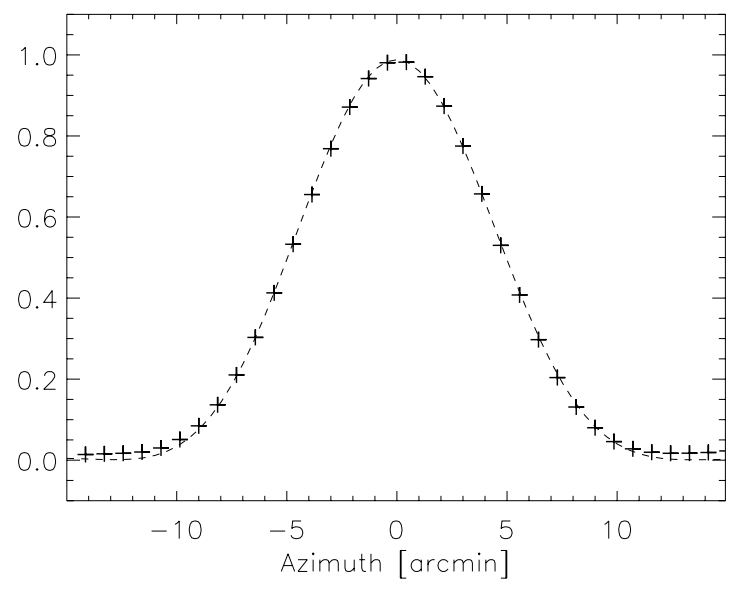

Fig. 15. Measurement (crosses) of the azimuth beam profile of channel $145 \mathrm{~W} 1$, obtained from repeated scans of a thermal source in the far field of the telescope. The measurements fit very well the prediction of the physical optics code BMAX (dashed line).

to measure the main beam, and a larger cylinder (with $76 \mathrm{~cm}$ diameter and $91 \mathrm{~cm}$ height) to map the sidelobes. The telescope was steered by a combination of a slow azimuth scan with a very slow elevation drift to map the beam profile of all the $\mathrm{B} 03$ detectors. The position of the source relative to the telescope was continuously measured with the tracking star camera. This system worked very well to measure 1D (azimuth) profiles of the beam. However, due to the short suspension chain used in the laboratory, the stability of the pointing system against pendulations was not good, and we estimate an uncertainty of $\gtrsim 10^{\prime}$ in the elevation measurements. For this reason we cannot estimate satisfactory 2D maps from the scans. We have verified that the data fit our physical optics (BMAX) beam model on single azimuth scans, as exemplified in Fig. 15, where $\chi^{2} /$ d.o.f. $\sim 1$. Similar results are obtained for many cuts of our beam, obtained at different elevation (which is left as a free parameter in the fits). So, even if we cannot measure the $2 \mathrm{D}$ beam directly from the ground calibration data, we are confident that we can use the beam computed from BMAX (plotted in Fig. 6) to model it at the required accuracy level. This is set by the presence of pointing jitter during the observations (see Sect. 5.1), at a level of $\gtrsim 2^{\prime}$ rms: in comparison, the small differences between the data and the best fit are negligible.

From the BMAX beam we compute the transfer function $B_{\ell}^{2}$ of the instrument in multipole space. This is plotted in Fig. 16, where it is also compared to a Gaussian beam transfer function. This $B_{\ell}^{2}$ has been convolved with the smearing resulting from pointing jitter, and used to deconvolve all our power spectrum measurements, as reported in Jones et al. (2006a), Piacentini et al. (2006), Montroy et al. (2006).

\section{Observations}

The instrument was launched by NASA-NSBF on Jan. 6, 2003, from Williams Field, near the McMurdo Station, in Antarctica ( $\left.167^{\circ} 5.760^{\prime} \mathrm{E} ; 77^{\circ} 51.760^{\prime} \mathrm{S}\right)$. The flight lasted until Jan. 21, with a total of $311 \mathrm{~h}$.

The altitude of the payload during the flight is reported in Fig. 17. The periodic variation is due to the daily change of elevation of the sun, while the long term trend is due to a small leak in the balloon. We released ballast, in a moderate amount on day 3 , and then in a full drop on day 5. After day 11, 


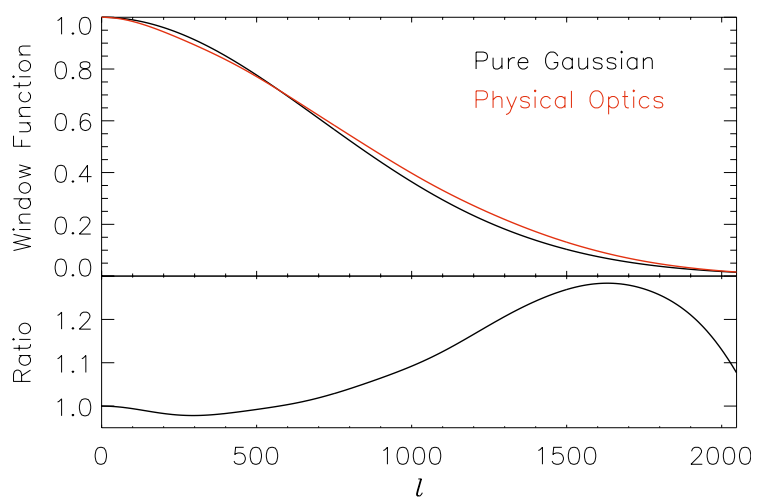

Fig. 16. Upper panel: instrument response at $145 \mathrm{GHz}$ in multipole space, computed from the BMAX physical optics model (red line). The beam is similar to a 9.8 arcmin $F W H M$ Gaussian (black line). The ratio between the window function for the actual beam and that for a Gaussian beam is plotted in the lower panel.

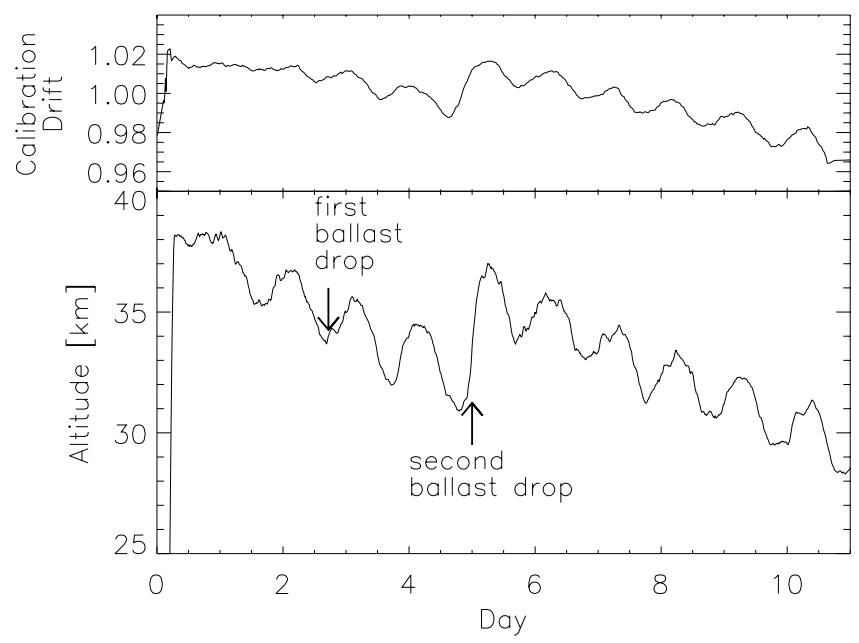

Fig. 17. Bottom: the altitude of the payload during the balloon-flight. The sinusoidal variation is due to the daily change of elevation of the sun, while the long term trend is due to a small leak in the balloon. The arrows indicate ballast releases used to counter the downward drift. Top: drift of detector responsivity during the flight as measured with the on-board calibration lamp: it is contained within a few \% for the whole length of the flight. The correlation with the altitude is due to to the variation of atmospheric pressure with altitude, which changes the temperature of the superfluid ${ }^{4} \mathrm{He}$ bath in the cryostat, with a consequent change in bolometer temperature.

the altitude dropped below $28 \mathrm{~km}$, telescope pointing become difficult, and we had to stop observations.

We devoted a total of $119 \mathrm{~h}$ to scans on the deep survey region, a total of $79 \mathrm{~h}$ to scans on the shallow survey region, and a total of $30 \mathrm{~h}$ to scans over the Galactic plane. The remaining hours have not been used for the data analysis, due to spurious signals after events like ballast drop or elevation changes, and due to testing, cryogenic operations, non optimal performance of the attitude control system at the lowest altitudes.

Maps of the sky coverage for the three surveys are reported in Figs. 18 and 19. The histogram of the integration time per pixel for the 3 different regions is plotted in Fig. 20.

\subsection{Attitude control system performance in flight}

Motion control was flawless in both the azimuth scanning and the drive of the inner frame elevation when the payload was at

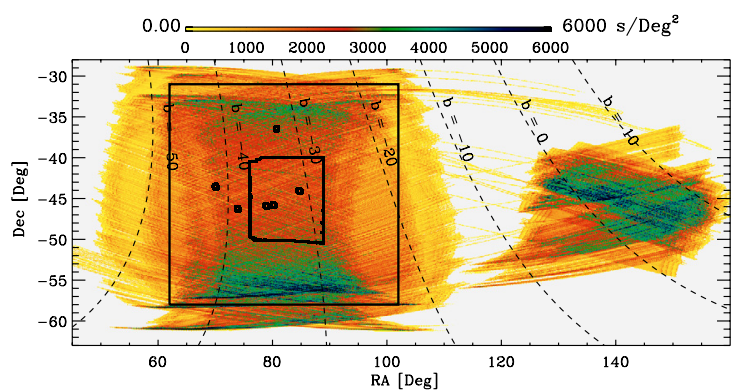

Fig. 18. Map of the observation time (integral over all the $145 \mathrm{GHz}$ bolometers) in the "Shallow" and "Galaxy" surveys of B03. The larger box includes pixels of the shallow survey actually used for the power spectrum analysis; the smaller box refers to the "Deep" survey (see Fig. 19).

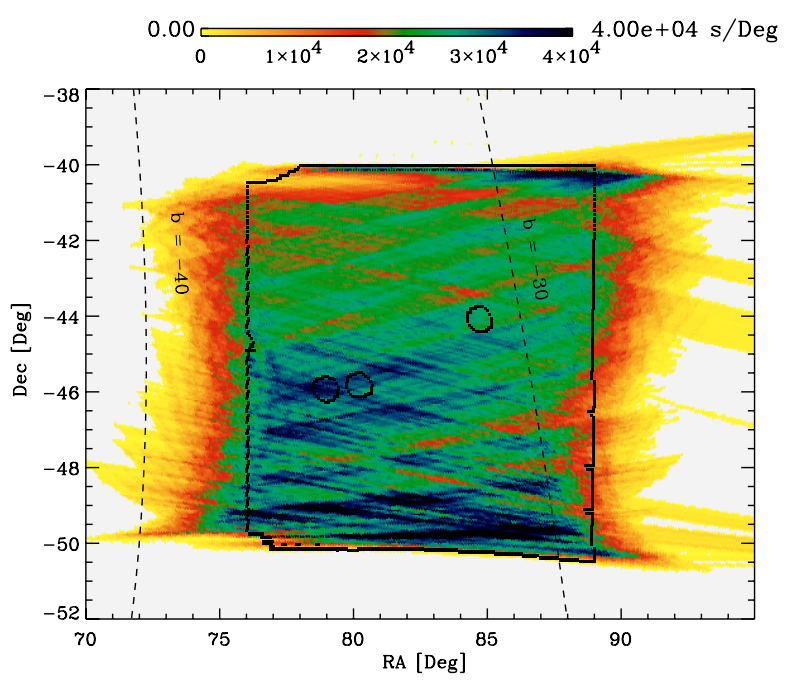

Fig. 19. Map of the observation time (integral over all the $145 \mathrm{GHz}$ bolometers) in the "Deep" survey of B03. This region overlaps completely with the central part of the shallow survey region. The box includes pixels of the deep survey actually used for the power spectrum analysis; however, the three circular regions marked around strong AGNs have been excised.

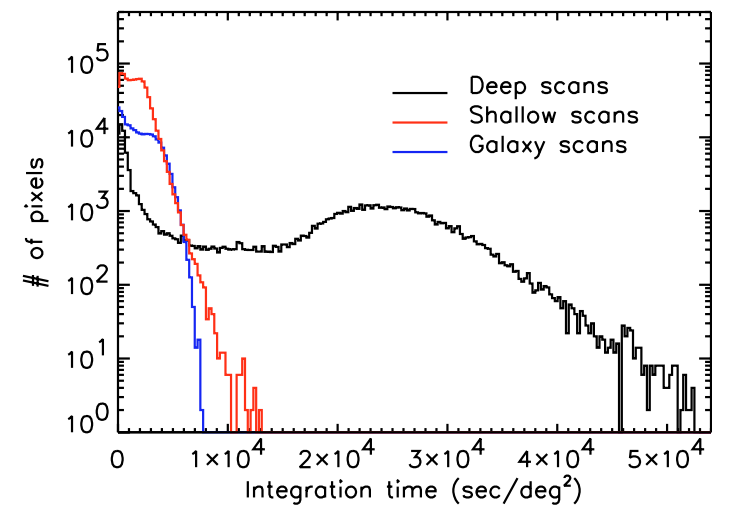

Fig. 20. Histogram of observation time per pixel (3.5' pixels) in the three surveys performed during the B03 flight.

nominal altitude ( $\gtrsim 25 \mathrm{~km}$ ). The GPS and FSS provided the required coarse, $\sim 10$ arcmin in-flight azimuth pointing and the gyroscopes functioned almost continuously, aside from short periods of dropouts or computer reboots. Near the end of the flight, once the payload altitude dropped below $\sim 23 \mathrm{~km}$, high winds made motion control impossible and the ACS was shut down. 
Performance of the tracking sensors, the SC and the PSS, was marred by communication problems in the ACS flight computer. With both sensors operating at the same time the computer was unable to parse the large amounts of incoming and outgoing data. To resolve this issue the PSS was turned off early in the flight. The SC provided the fine pointing data for the first and last thirds of the flight. The cold temperature at low (pre-ballast drop) altitude on day 4 caused the SC to freeze up, at which point the PSS was turned back on, providing fine pointing for the middle third of the flight.

\section{Raw data processing}

The polarization signal we are looking for is very small. In the observations of the deep region at $145 \mathrm{GHz}$ the contribution from CMB polarization is of the order of a thousandth of the noise for a single signal sample, and of the order of a tenth of the error for the final measurement of a single $3.5^{\prime}$ pixel. For this reason we must properly exclude instrumental artifacts and avoid the introduction of bias. We developed two completely independent analysis pipelines, which will be called IT (Italy) and NA (North America) in the following discussion. In this section and in the following we outline the data analysis and specify where the two pipelines make different choices. Despite the many differences, the final spectra and maps are fully consistent. This is the best test of the robustness of the dataset against alternative data selection and analysis procedures.

The raw data acquired by the B03 telescope is convolved with a transfer function consisting of the thermal response of the detector and the filtering of the readout electronics. It also contains transients caused by cosmic rays and our calibration lamp, and longer time-scale events caused by thermal instabilities resulting after each elevation change. At each step of the data cleaning, we flag (mark as bad) some samples in the datastream and fill these flagged samples with a constrained realization of the noise. This noise filling uses linear prediction to replace flagged samples based on the unflagged data on either side. After removing very large cosmic rays, we flag a minimal number of samples around each elevation change and fit out a decaying exponential to the signal recovery.

Next, the datastreams are deconvolved with the combined thermal and electrical transfer functions. For the NA pipeline, we used transfer functions determined by pre-flight lab measurements (described in Sect. 3.1). For the IT pipeline we used the in-flight determination of the transfer function described in Sect. 6.1.

The samples containing signals from our calibration lamp are flagged. Medium amplitude cosmic rays are then flagged by passing a simple template through the timestream and looking for spikes larger than a certain size. The data is then heavily band-pass filtered to make small cosmic rays more visible. A second template is used to flag these small cosmic rays. This process might mistake bright galactic sources for small cosmic rays. To avoid this, we make a list of all bright source crossings in our datastreams by using the coincidence of sources in both detectors of a pixel pair. These samples are explicitly unflagged. After building up a list of flagged samples, these samples are filled with noise in the deconvolved data.

After completing this primary data cleaning, we found that each of our time-streams contained an extraneous component that was well correlated with the accelerations of the telescope's pitch and roll gyroscopes. We fit these accelerations to the data in hour long chunks and subtracted this contribution from the time-streams.

\subsection{Post-flight attitude determination}

Pointing reconstruction consists of determining where each detector was pointing in the sky at each data sample. We had a redundant number of attitude sensors that can be combined in different ways to obtain the final pointing solution. The two pipelines are significantly different in this respect.

\subsection{IT pointing reconstruction}

Pointing is described by the rotation matrix $S$ that transforms from the Celestial reference frame to the telescope reference frame. In addition, the constant offsets of the pointing directions of the horns in the telescope frame must be determined. The problem is separated into two parts $S=A \cdot B$, where the rotation matrix $A$ converts from an Earth local reference frame to the telescope frame, and $B$ is the astronomical conversion. $A$ is called the attitude matrix and is defined by three Euler angles. It can also conveniently be described by a quaternion.

To derive the attitude matrix we use several attitude sensors, measuring either absolute angles or angular velocities. In our case the absolute sensors are a differential GPS, a Stellar Sensor and two Solar Sensors. The differential GPS can by itself measure the attitude matrix $A$, but suffers for low frequency drifts,

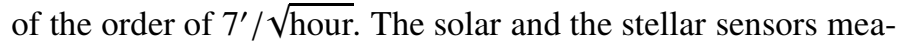
sure the sensor-Sun or sensor-Star angles in the payload frame, each providing two equations for the three unknown Euler angles. Their combination thus provides a solvable system. The angular velocities are obtained from the laser gyroscopes, which have a bias due to their orientation in the Earth's magnetic field.

We combine the signals of the different sensors taking into account their noise/drift properties, by means of an optimized Kalman filter (Kalman 1960) and by properly flagging the sensors signals. The Kalman filter is based on the propagation from the status at time $t$ to the status at time $t+\Delta t$ as predicted by the dynamic measurements (from gyroscopes), in combination with the direct measurements of the status $t+\Delta t$ (update). The weighting of the update is given by the noise properties of the gyroscopes and of the sensors.

The direction of lines of sight of the different detectors in the telescope frame are derived from observation on known sources, i.e. AGNs and Galactic HII regions.

A final correction to the IT pointing solution is obtained by dividing the timestream in hour-long chunks. For each chunk we make a sky map, and find the Azimuth and elevation offsets which best fit a single template map derived from the BOOMERanG data from the 1998 flight. An IRAS template was used for the scans on the Galactic plane. The pointing solution is then corrected using these best fit offsets, which are of the order of $\lesssim 1$ arcmin.

The accuracy of the IT pointing solution is tested by means of the signals detected in the direction of bright AGNs (see Sect. 6.2), and is of the order of $(2.4 \pm 0.3)^{\prime} \mathrm{rms}$.

\subsection{The NA pointing solution pipeline}

Spikes in the raw pointing data are removed, and where possible (over a few samples) the data can be linearly interpolated. Postflight re-calibrations are applied to the SC, PSS and FSS data. For the SC/PSS azimuth and elevation this is a simple matter of rotating the coordinates through a small angle until correlated signal is minimized. The FSS is re-calibrated using azimuth data from the GPS and the SC. A look-up table of sun elevation versus raw FSS azimuth is constructed. Each element of the table 
contains a GPS/SC derived sun azimuth relative to the gondola, averaged over the whole flight. Raw FSS data is replaced by the corresponding element in the table. Another useful derived quantity is obtained from the GPS up, north and east velocity data. The relatively stable LDB environment allows one to model the gondola as a pendulum. With this model first estimates of gondola pitch and roll, pitch $G V$ and roll $_{G V}$ may be calculated from the GPS velocity data.

Clean, calibrated pointing fields are combined to determine the gondola attitude. The sensors used in the final analysis include the FSS azimuth, the SC elevation, pitch $_{G V}$, roll $_{G V}$ and the integrated azimuth, pitch and roll gyro data. At frequencies below $\sim 50 \mathrm{mHz}$ the pointing solution is based on the best fit azimuth, pitch and roll to the sun and star positions as determined by the star camera, the FSS, pitch ${ }_{G V}$ and $\operatorname{roll}_{G V}$. Gaps in the pointing data less than $\sim 40 \mathrm{~s}$ long are filled with integrated gyro data and above $\sim 50 \mathrm{mHz}$ the pointing solution is strictly gyro signal. Gaps longer than $\sim 40 \mathrm{~s}$ are flagged.

The elevation encoder signal is added to the gondola pitch, thereby translating gondola (outer frame) attitude into telescope (inner frame) attitude. The beam offsets for each detector are obtained from fits to the five brightest QSOs in the CMB field. Galactic and CMB source centroid offsets reveal a $0.1^{\circ}$ shift in gondola pitch after the mid-flight ballast drop. To account for this approximately $6 \mathrm{~h}$ of data during and after the ballast drop are flagged and a pitch shift is applied to all pointing data preceding the drop. The reconstructed elevation and azimuth of each beam on the sky, along with the measured polarization angles for each detector, and the GPS latitude, longitude and time are combined to determine the right ascension, declination and the angle between the principal axis and the meridian for each beam.

The CMB field pointing error, based on comparisons of analytical beams with observed beams, is $\sim 2.5^{\prime} \mathrm{rms}$ in azimuth and $\sim 1.5^{\prime} \mathrm{rms}$ in elevation (see Sect. 6.2).

\section{In-flight calibration}

\subsection{Transfer function}

The transfer function of each detector, including the readout, has been measured in flight using a procedure similar to the one described in Crill et al. (2003). Even though the detectors are designed to minimize cosmic rays cross section, each detector produces detectable cosmic ray hits at the rate of about one every two minutes, leaving on the data-stream a typical signature which is the response of the system to an impulsive input. This signature is the transfer function of the system in real space.

Of all the cosmic rays events in a given channel, only the subset producing a spike in the (0.5-4) Volts range is selected for this analysis, in order to neglect the effects of noise and to avoid saturated events. Each event in the database is shifted in time and normalized to minimize the chi square to a first order approximation of the impulse response. The shift is performed on a grid much finer than the $60 \mathrm{~Hz}$ sampling rate of the Data Acquisition System. The combination of all the hits provides a template of the impulsive response of each detector. As an example, we plot in Fig. 21 the data for detector 145W1. The result is insensitive to the choice of the first order approximation. The Fourier transforms of those templates are the transfer functions of the detectors. The method is sensitive in a frequency range between $\sim 0.1$ and $\sim 250 \mathrm{~Hz}$. The resulting transfer function is similar (but not exactly equal) to the one described in Sect. 3.1. The small differences are probably due to the internal time constant of the detector absorber, which affects in different ways the

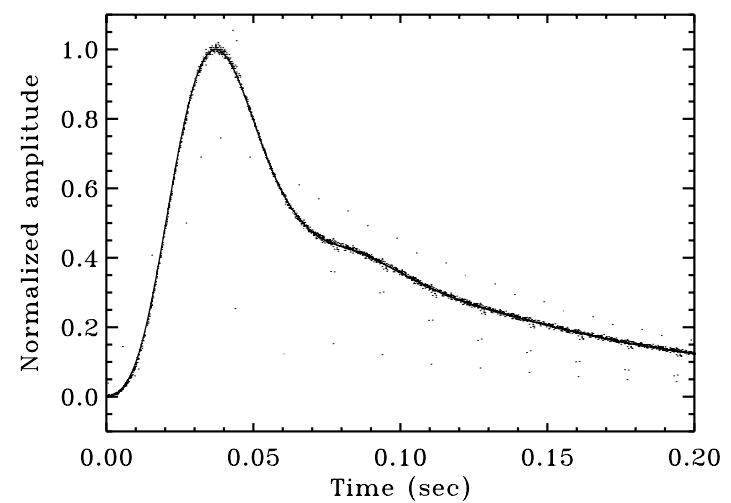

Fig. 21. In-flight response of the $145 \mathrm{~W} 1$ channel to an impulsive event. The frequency response of the system is the Fourier Transform of this response. The points are accumulated from several cosmic-rays events shifted and normalized to fit the same template.

response to mm-wave photons (depositing their energy on the entire absorber) and the response to particles (depositing their energy on a small localized part of the absorber). The IT pipeline uses this transfer function, complementing it with lab calibration data (Sect. 3.1) at $f<0.1 \mathrm{~Hz}$. The NA pipeline uses the transfer function derived from pre-flight measurements.

\subsection{Effective beam calibration}

The effective $\mathrm{B} 03$ beam profiles are estimated combining physical optics modeling and observations of bright extragalactic point sources.

The physical beam is computed with a physical optics simulation of the telescope (see Sect. 2.6 and Fig. 15); its shape is similar to a Gaussian function with a $F W H M$ of 9.8 arcmin for the $145 \mathrm{GHz}$ channels and of 5.6' for the 245 and $345 \mathrm{GHz}$ channels, with residuals of the order of $2 \%$ (see Fig. 16).

Pointing errors produce a jitter on the map that we assumed to be Gaussian and uniform in all the observed sky regions. Under this assumption the jitter is defined by a single parameter, $\sigma_{\text {jitter. }}$.

The observed profile of a point source is the convolution of the physical beam with the Gaussian describing the pointing jitter. For a Gaussian and isotropic physical beam we would have

$B\left(\theta, \sigma_{\text {jitter }}\right)=\exp \left[-\frac{\theta^{2}}{2\left(\sigma_{\text {beam }}^{2}+\sigma_{\text {jitter }}^{2}\right)}\right]$.

Using the actual physical beam gives a result very similar to Eq. (24). We measured $\sigma_{\text {jitter }}$ by comparing the expected beam to the profiles of five sources derived from the 8 PSBs maps at $145 \mathrm{GHz}$. We did not use the higher frequency channels to estimate the jitter because of their higher noise. The jitter, estimated as the weighted average of the 5 best fit values, is $\sigma_{\text {jitter }}=(2.5 \pm 0.3)$ arcmin. The $F W H M$ of the effective beam used in the final analysis is $(11.2 \pm 0.3)$ arcmin for the $145 \mathrm{GHz}$ channels, $(7.8 \pm 0.3)$ arcmin for the 245 and $345 \mathrm{GHz}$ channels.

\subsection{Gain drift from calibration lamp}

The responsivity of the bolometers depends on the operating temperature and on the radiative loading. Both these quantities can change during the flight.

For this reason, we include a calibration lamp in the cold optics of BOOMERanG, that flashes every $\sim 15$ min during the flight. 
Details on the lamp, which is a composite bolometer structure suspended in the center of a $1 \mathrm{~cm}$ hole in the tertiary mirror, are in Crill et al. (2003). Its intrinsic stability depends on the fact that the temperature achieved during the flashes is significantly higher than the $2 \mathrm{~K}$ base temperature. In this limit, the emission of the lamp depends only on the heater current, which is stable to better than $0.1 \%$.

The amplitude of the calibration signal changes only by a few percent during the flight, as shown in the top panel of Fig. 17. To demonstrate that these fluctuations are not intrinsic to the source, we have analyzed the DC signal across the bolometers. This is proportional to the resistance of the bolometers, which in turn is proportional to the responsivity. For all the detectors we found a very good correlation between the bolometer responsivity estimated from the calibration lamp and the DC level: a clear indication that the signal of the calibration lamp is constant through the flight.

\subsection{Bolometer performance}

We compute in-flight bolometer parameters with a bolometer model based on Holmes et al. (1998), Jones (1953), and Mather (1982) (see Jones 2005 for details). The receiver model computes the total optical load on the detectors using the resistance and thermal conductivity as functions of temperature measured on the ground, and the in-flight DC bolometer voltage, bias current, and noise.

We further model the optical load on the detectors due to the atmosphere, telescope, and Cosmic Microwave Background, and assume that leftover optical load is due to internal parasitic loading. The optical load and responsivity change by several percent during the flight (see Sect. 6.3) due to changes in emission from the atmosphere and thermal radiation from cryostat components.

We model the contributions to the noise from photon shot noise, Johnson noise, phonon shot noise in the thermal link between the bolometer and the cold stage, and amplifier noise and compute the NEP (Noise Equivalent Power) contributed by each. The results of these calculations, for typical values of the inflight optical load, are reported in Table 5.

A source of impulsive noise is cosmic rays. All the B03 detectors have been designed to minimize their cross section to cosmic rays. As a result, even in the polar stratosphere, the average rate of cosmic-rays hits in PSBs is one every two minutes. Over all the identified events, $83 \%$ produce transients with amplitude at the ADC $0.05<A<1 V ; 10 \%$ have amplitude $1 V<A<2 V, 5 \%$ have $2 V<A<4 V$ and $3 \%$ have $A>4 V$. All these events are flagged and removed from the analysis as explained in Sect. 5.

\subsection{Noise estimation}

The measurement of the actual in-flight noise is obtained via a Fourier transform of the raw datastream. A sample power spectrum of the raw data (deconvolved from system frequency response, as estimated in Sects. 3.1 and 6.1) is shown in Fig. 22. Signal contributions are present above the noise, at the scan frequency and at its harmonics, and in the $\sim 0.1 \mathrm{~Hz}$ range. The Generalized Least Squares (GLS) map-making method relies on knowledge of the noise correlation function, which is related to the noise power spectral density by a Fourier transform. This quantity must be estimated from the data themselves, which are a combination of noise and signal.
Table 5. The receiver performance for a typical channel from each of the BOOMERANG bands, computed from a receiver model using the observed average in-flight loading conditions. The performance measured in flight is consistent with these estimates.

\begin{tabular}{lcrrr}
\hline \hline & units & 145 & 245 & 345 \\
\hline Optical power & $\mathrm{pW}$ & 0.496 & 0.846 & 6.158 \\
Equivalent R-J temperature & $\mathrm{K}$ & 4 & 4 & 16 \\
$\quad \mathrm{CMB} /$ total & $\%$ & 23.0 & 6.2 & 0.9 \\
Atmos./total & $\%$ & 4.1 & 32.6 & 64.7 \\
$\quad$ Refl./total & $\%$ & 21.4 & 17.2 & 10.0 \\
$\quad$ Internal/total & $\%$ & 51.5 & 44.0 & 24.4 \\
\hline $\mathrm{NEP}_{\text {total }}$ & $10^{-17} \mathrm{~W} / \sqrt{\mathrm{Hz}}$ & 2.49 & 3.89 & 8.95 \\
$\mathrm{NEP}_{\text {photon }}$ & $10^{-17} \mathrm{~W} / \sqrt{\mathrm{Hz}}$ & 0.99 & 1.64 & 5.31 \\
$\quad$ Bose term & $\%$ & 14.8 & 7.2 & 16.4 \\
$\mathrm{NEP}_{\text {phonon }}$ & $10^{-17} \mathrm{~W} / \sqrt{\mathrm{Hz}}$ & 1.003 & 1.636 & 2.891 \\
$\mathrm{NEP}_{\text {johnson }}$ & $10^{-17} \mathrm{~W} / \sqrt{\mathrm{Hz}}$ & 1.307 & 1.839 & 3.669 \\
$\mathrm{NEP}_{\text {amplifier }}$ & $10^{-17} \mathrm{~W} / \sqrt{\mathrm{Hz}}$ & 1.578 & 2.518 & 5.494 \\
$\mathrm{NEP}_{\text {bolo }} / \mathrm{NEP}_{\text {background }}$ & & 1.67 & 1.50 & 0.88 \\
\hline $\mathrm{NEFD}_{\mathrm{NET}_{\text {cmb }}}$ & $\mathrm{mJy} / \sqrt{\mathrm{Hz}}$ & 37 & 43 & 177 \\
$\mathrm{NET}_{\mathrm{RJ}}$ & $\mu \mathrm{K}_{\mathrm{CMB}} \sqrt{\mathrm{s}}$ & 151 & 341 & 551 \\
$\mathrm{NET}_{\text {cmb }}$ & $\mu \mathrm{K}_{\mathrm{RJ}} \sqrt{\mathrm{s}}$ & 89 & 92 & 51 \\
\hline Voltage noise & $\mu \mathrm{K}_{\mathrm{CMB}} \sqrt{\mathrm{s}}$ & 53 & 171 & 275 \\
\hline & $\mathrm{nV} / \sqrt{\mathrm{Hz}}$ & 18.0 & 17.7 & 18.5 \\
\hline
\end{tabular}

The standard way to characterise the underlying noise is by subtracting an estimate for the signal (see Ferreira \& Jaffe 2000). Since the signal can be estimated by making a map, the two problems are entangled, and a possible approach is iterative (see Prunet et al. 2001 and Dore et al. 2001; see however Natoli et al. 2002 for an alternative approach).

In the IT pipeline, a rough estimate of the signal is obtained by naïvely coadding a band-pass filtered version of the timeline; the resulting map is used as a baseline to obtain an estimate of the noise power spectral density. This is in turn used to make a new, GLS, map and the process can be iterated as desired. We have found empirically that, for $\mathrm{B} 03$, no significant improvement is obtained by iterating more than five times. Furthermore, tests on simulated data suggest that, under these conditions, the underlying noise properties are recovered without any substantial bias (see De Gasperis et al. 2005).

The following scheme is employed to optimally use the information produced by all detectors. The above procedure is repeated for each uncalibrated bolometer timeline (e.g. eight times, one for each $145 \mathrm{GHz}$ bolometer). Then, a first estimate of the relative calibration factors between channels at a given frequency are computed as explained in Sect. 7.2.1. A multichannel, high signal to noise ratio, relatively calibrated map is produced and used as an estimate for the underlying signal to evaluate a more precise noise power spectral density for each bolometer. As a further step, relative calibration factors are recomputed with these noise estimates, and a new multichannel map is made. We have verified that iterating further is useless since if this last map is used to produce further noise estimates, the latter do not change significantly.

A sample noise power spectrum is shown in Fig. 22. With this method we estimate the noise of all the B03 channels in $V / \sqrt{\mathrm{Hz}}$. This is converted into NEP (see Table 6) using the measured in-flight responsivity (see Sects. 7.2.1 and 7.2.2).

There is a component of noise correlated between different detectors. This is especially visible at very low frequencies. In Fig. 22 we include an example. This noise has a similar level for all detector couples (roughly independent of whether the 


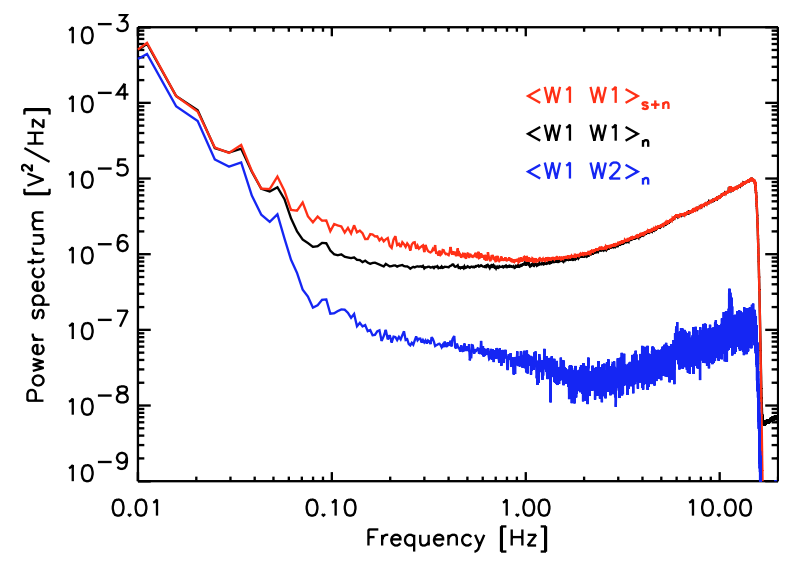

Fig. 22. Flight-average of the auto power spectrum of the data timestream, from channel 145W1 (top line, red). The data are recorded at the ADC input. The total gain of the readout chain between the bolometer and the ADC input is 50000 . The middle line (black) is the auto power spectrum of the time stream after subtraction of the signal estimated from the best fit map: this is an estimate of the instrumental noise. Comparing the two, it is evident that most of the CMB signal is encoded in the $0.05-1 \mathrm{~Hz}$ range. The lowest line (blue) is the cross correlation of the noise (time-stream - best fit map) signal from 145W1 and $145 \mathrm{~W} 2$. In the frequency range of interest for the CMB signal, the crosscorrelated noise signal is at least one order of magnitude smaller than the auto-correlated signal. The correlated noise rises at very low frequencies, where atmospheric effects, thermal drifts, pendulations and scan-related microphonics can be present. At frequencies higher then $2 \mathrm{~Hz}$ the noise correlation remains well below the noise of the detector.

Table 6. In flight detectors performance: we report the noise equivalent temperature at $1 \mathrm{~Hz}$, derived from the noise equivalent voltage measured in flight as in Sect. 6.4, from the responsivity estimated as in Sect. 7.2.1 and from the spectral calibrations of Sect. 3.2. In our survey, this frequency corresponds to multipoles $400 \lesssim \ell \lessgtr 1200$. The noise increases at lower and at higher frequencies, as shown in Fig. 22.

\begin{tabular}{cc}
\hline \hline Channel & NET $\left(\mu K_{\mathrm{CMB}} \sqrt{\mathrm{s}}\right)$ \\
\hline $145 \mathrm{~W} 1$ & 140 \\
$145 \mathrm{~W} 2$ & 137 \\
$145 \mathrm{X} 1$ & 156 \\
$145 \mathrm{X} 2$ & 150 \\
$145 \mathrm{Y} 1$ & 151 \\
$145 \mathrm{Y} 2$ & 159 \\
$145 \mathrm{Z} 1$ & 182 \\
$145 \mathrm{Z} 2$ & 281 \\
\hline $245 \mathrm{~W}$ & 281 \\
$245 \mathrm{X}$ & 358 \\
$245 \mathrm{Y}$ & 316 \\
$245 \mathrm{Z}$ & 331 \\
\hline $345 \mathrm{~W}$ & 459 \\
$345 \mathrm{X}$ & 424 \\
$345 \mathrm{Y}$ & 620 \\
$345 \mathrm{Z}$ & 309 \\
\hline
\end{tabular}

detectors are members of the same PSB pair). It may originate in scan synchronous microphonics and in fluctuations of the residual atmosphere. The levels of correlated noise in the interesting frequency band $(0.1-2 \mathrm{~Hz})$, for all possible couples of $145 \mathrm{GHz}$ bolometers, are compared to the levels of noise of each bolometer in Table 7. The presence of this correlated noise is neglected in the map-making, but is taken into account for our estimates of the power spectra (see Jones et al. 2006a; Piacentini et al. 2006; Montroy et al. 2006).
Table 7. Absolute value of the noise cross-power-spectrum for the $145 \mathrm{GHz}$ detectors, averaged in the $0.1-2 \mathrm{~Hz}$ range. The numerical values have been normalized to the noise auto-power-spectrum of detector $145 \mathrm{~W} 1$, which is $1.2 \times 10^{-6} \mathrm{~V}^{2} / \mathrm{Hz}$ at the ADC input.

\begin{tabular}{ccccccccc}
\hline \hline & $\mathrm{W} 1$ & $\mathrm{~W} 2$ & $\mathrm{X} 1$ & $\mathrm{X} 2$ & $\mathrm{Y} 1$ & $\mathrm{Y} 2$ & $\mathrm{Z} 1$ & $\mathrm{Z} 2$ \\
\hline $\mathrm{W} 1$ & 1.000 & & & & & & & \\
$\mathrm{~W} 2$ & 0.060 & 0.850 & & & & & & \\
$\mathrm{X} 1$ & 0.034 & 0.031 & 1.100 & & & & & \\
$\mathrm{X} 2$ & 0.033 & 0.031 & 0.059 & 1.000 & & & & \\
$\mathrm{Y} 1$ & 0.048 & 0.040 & 0.035 & 0.035 & 1.200 & & & \\
$\mathrm{Y} 2$ & 0.043 & 0.036 & 0.035 & 0.035 & 0.016 & 1.200 & & \\
$\mathrm{Z} 1$ & 0.037 & 0.033 & 0.038 & 0.037 & 0.041 & 0.041 & 1.500 & \\
$\mathrm{Z} 2$ & 0.060 & 0.054 & 0.063 & 0.061 & 0.066 & 0.066 & 0.096 & 4.300 \\
\hline
\end{tabular}

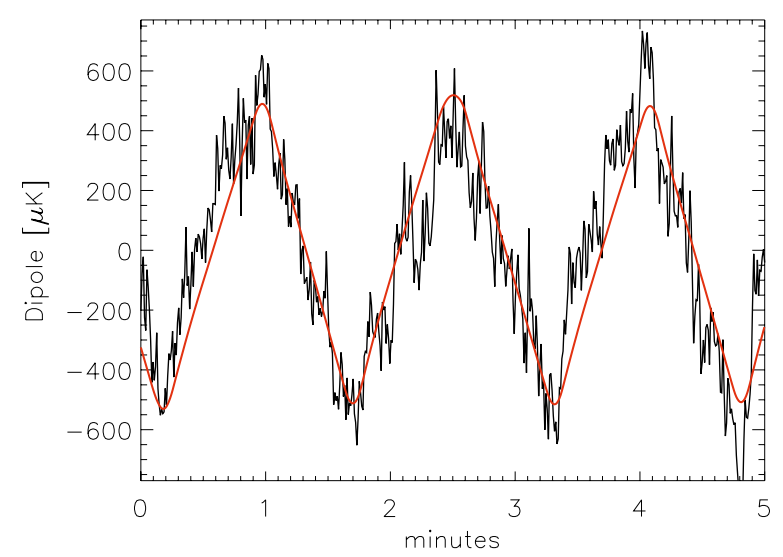

Fig. 23. Raw-data time-stream from channel $145 \mathrm{~W} 1$. It is evident the scan-synchronous signal from the CMB dipole. The (red) line is the CMB dipole, measaured by WMAP and COBE, along this scan. There is clear agreement between the predicted CMB dipole and the observed scan synchronous signal.

\subsection{CMB dipole}

The dipole of the CMB is visible as an approximately linear drift along our short scans. It produces a scan-synchronous triangle wave (see Fig. 23) that we have removed from the timestreams before proceeding with the analysis. Since it is scansynchronous, as some possible systematics, we cannot use it for a precise calibration of the instrument: the calibration accuracy achievable with the dipole is $\gtrsim 15 \%$, not sufficient for our purposes. However, its amplitude is consistent with the amplitude of the CMB Dipole measured by COBE and WMAP.

\section{Stokes parameters maps}

\subsection{The map making pipeline}

A Generalized Least Square (GLS) method is used to jointly estimate the Stokes parameter sky maps $I, Q$ and $U$ from time ordered data. The best maps are obtained by combining data from all detectors available at a given frequency. This approach is implemented in the ROMA map making code (De Gasperis et al. 2005) for the IT pipeline, and in the DIQU code (Jones et al. 2006b) for the NA pipeline. Following Eq. (10), we assume the following data model:

$\mathcal{D}_{t}^{i}=\frac{\mathcal{S}^{i}}{2} A_{t p}^{i}\left[I_{p}+\frac{1-\epsilon_{i}}{1+\epsilon_{i}}\left(Q_{p} \cos 2 \alpha_{t}^{i}+U_{p} \sin 2 \alpha_{t}^{i}\right)\right]+n_{t}^{i}$.

Here $i, t$ and $p$ label channel, time and map pixel respectively; $D_{t}^{i}$ are the time ordered data for a given channel, related to the 
sky maps $I_{p}, Q_{p}$ and $U_{p}$ by the pointing operator $A_{t p}^{i}$, which we assume to be as simple as possible: its elements are equal to one when a pixel is observed, and are zero otherwise. Equation (25) can be recast in a more compact formalism by defining a generalized pointing matrix $\mathbf{A}_{t p}$ which includes the trigonometric functions and a map triplet $\mathbf{S}_{p} \equiv\left(I_{p}, Q_{p}, U_{p}\right)$ :

$\mathcal{D}_{t}=\mathbf{A}_{t p} \mathbf{S}_{p}+\mathbf{n}$.

The GLS estimator for $\mathbf{S}_{p}$ is then (see De Gasperis et al. 2005)

$\widetilde{\mathbf{S}}_{p}=\left(\mathbf{A}^{t} \mathbf{N}^{-1} \mathbf{A}\right)^{-1} \mathbf{A}^{t} \mathbf{N}^{-1} \mathcal{D}$,

where $\mathbf{N}$ is the noise covariance matrix, whose estimation is discussed in Sect. 6.5 above:

$\mathbf{N} \equiv\left\langle\mathbf{n}_{t} \mathbf{n}_{t^{\prime}}\right\rangle=\left(\begin{array}{ccc}\left\langle n_{t}^{1} n_{t^{\prime}}^{1}\right\rangle & \cdots & \left\langle n_{t}^{1} n_{t^{\prime}}^{n}\right\rangle \\ \vdots & \ddots & \vdots \\ \left\langle n_{t}^{n} n_{t^{\prime}}^{1}\right\rangle & \cdots & \left\langle n_{t}^{n} n_{t^{\prime}}^{n}\right\rangle\end{array}\right)$.

This matrix becomes block diagonal assuming that there is no noise correlation between different detectors:

$\left\langle n_{t}^{i} n_{t^{\prime}}^{j}\right\rangle=\left\langle n_{t}^{j} n_{t^{\prime}}^{i}\right\rangle=0 \quad(i \neq j)$.

Given the size of the problem, in the IT pipeline the map making normal Eq. (27) are solved by implementing a Fourier-based preconditioned conjugate gradient iterative solver, which only needs to perform matrix to vector products. This approach critically depends on the assumption that the noise is stationary (Natoli et al. 2001). In the NA pipeline a Jacobi solver is used.

In practice, there are many further details. Since the solver works in Fourier space, the timeline must be continuous; bad or missing data chunks ("gaps") are replaced by a constrained realization of Gaussian noise (Hoffman \& Ribak 1991), designed to mimic the correct noise behavior at the gap's boundaries. The samples belonging to these chunks are then flagged and the solver is instructed not to take them into account, as they do not contain any useful data. The noise correlation functions (also called noise filters) are band pass filtered in the range $70 \mathrm{mHz}-14.5 \mathrm{~Hz}$ before being given to the map making code.

Each detector has its own noise filter, which is kept constant over the full flight (i.e. data are reduced as if the noise were stationary within a single detector) for the IT pipeline, while the NA pipeline estimates noise separately for each ( $\sim$ hour long) chunk of data. The NA approach treats the non stationarity of the data, at the cost of a reduced accuracy in the estimate of the noise filter. The differences in the resulting maps are negligible.

For the polarization maps, detectors forming PSB pairs are treated as independent detectors in the IT pipeline, while the difference of their signals is used by the NA pipeline (see Jones et al. 2006b, for details).

The ROMA code employs about 20 min (and 200 iterations) to produce a set of $I, Q, U$ maps from an eight bolometer (full flight) timeline, when running a 128 processor job on an IBM SP machine featuring $450 \mathrm{MHz}$ Power 3 processors.

\subsection{Responsivity calibration}

The first step in obtaining our final product maps of $I, Q, U$, is the relative calibration of all the detectors. As explained in Sect. 2.1, we need a relative calibration accuracy of the order of $\$ 2 \%$. We start by producing single detector maps of I using one of the map-making procedures. We then proceed in two ways: in pixel space and in multipole space.
Table 8. Relative calibration $\mathcal{R}$ of the PSB channels obtained from the pixel-pixel scatter plots with NSIDE $=256$ (second column, see Sect. 7.2.1) and from the cross-spectrum (third column, see Sect. 7.2.2), using $W_{1}$ as the reference channel. For $\mathcal{R}_{* W_{1}}^{\text {spectrum }}, 1-\sigma$ errors are used. For $\mathcal{R}_{* W_{1}}^{\text {pixel }}$, we also include in the error the possible bias due to a conservative $\pm 20 \%$ error in the estimate of the noise.

\begin{tabular}{ccc}
\hline \hline channel & $\mathcal{R}_{* W_{1}}^{\text {pixel }}$ & $\mathcal{R}_{* W_{1}}^{\text {spectrum }}$ \\
\hline $145 W 2$ & $0.956 \pm 0.028$ & $0.9391 \pm 0.0023$ \\
$145 X 1$ & $0.985 \pm 0.029$ & $0.9498 \pm 0.0025$ \\
$145 X 2$ & $0.749 \pm 0.022$ & $0.7300 \pm 0.0022$ \\
$145 Y 1$ & $0.920 \pm 0.030$ & $0.9177 \pm 0.0024$ \\
$145 Y 2$ & $0.912 \pm 0.029$ & $0.8918 \pm 0.0030$ \\
$145 Z 1$ & $0.674 \pm 0.021$ & $0.6702 \pm 0.0019$ \\
$145 Z 2$ & $0.407 \pm 0.023$ & $0.4183 \pm 0.0018$ \\
\hline
\end{tabular}

\subsubsection{Relative calibration from pixel-pixel correlation of different PSBs}

We carry out this analysis for the PSBs in the deep region. We use the $145 \mathrm{GHz} W_{1}$ channel as the reference channel. The characteristics of these channels (beam, frequency response, noise, polarization efficiency) are so similar that their data can be directly compared, pixel to pixel. For channel $j$ we scatter plot the $I_{i, j}$ values (where $i$ is the pixel index) versus the $I_{i, W_{1}}$ values, and we fit the data to a straight line. We estimate the error on each $I_{i, j}$ as $\sigma_{j} / \sqrt{N_{j, i}}$ where $\sigma_{j}$ is obtained by integrating the TOD noise power spectrum, and $N_{j, i}$ is the number of observations of pixel $i$. The slope of the best fit line is the relative calibration factor $\mathcal{R}_{j, W_{1}}$. Simulations show that this procedure results in an accurate (within 1\%) estimate of the relative calibration. The results for all channels are reported in Table 8.

We also study the effect of a mis-estimate of the noise in the responsivity estimate. This depends strongly on the signal to noise per pixel of the data. For $7^{\prime}$ pixels, the signal to noise per pixel for CMB anisotropies is $\sim 3$. In these conditions, simple simulations show that in order to bias the relative calibration by more than $2 \%$ the estimate of the noise must be wrong by more than $20 \%$. We conclude that this method provides a robust estimate of the relative calibration. The errors on the relative calibration results reported in Table 8 include the effect of a conservative $20 \%$ misestimate of the errors per pixel.

If, instead, the $\mathrm{S} / \mathrm{N}$ per pixel is $\lesssim 1$, then a $20 \%$ misestimate of the noise induces $\gtrsim 20 \%$ errors in the calibration. This happens when an absolute calibration is attempted by fitting $I_{i, j}$ versus the $I_{i \text {,WMAP. }}$ Since the angular resolution of WMAP is worse than the one of $\mathrm{B} 03$, and the noise per pixel is higher, the slope of the best fit line is significantly biased. Also, working in pixel space it is not trivial to take into account the difference in beam size and shape between B03 and WMAP.

\subsubsection{Absolute and relative calibration from ratios of cross-spectra}

We can compare the signals detected by different detectors observing the same sky in multipole space rather than in pixel space. A cross-power spectrum is very useful in this case, due to its unbiased nature: the noise properties of the two signals do not affect the results. Moreover, the use of the cross-power spectrum allows us to properly take into account in a simple way the effect of the different angular resolution, and map-making transfer functions. All these effects can be included in a function $F_{\ell}^{k}$, which can be different for each channel $k$. Since we do see CMB fluctuations in all the B03 maps, this method can be used to 
calibrate the $\mathrm{B} 03$ detectors at 145,245 and $345 \mathrm{GHz}$, and to find the absolute calibration factor by comparing $\mathrm{B} 03$ maps to a reference map from WMAP.

We expand the uncalibrated maps of all channels in spherical harmonics:

$V_{k}=\sum_{\ell, m} a_{\ell, m}^{k} Y_{m}^{\ell} F_{\ell}^{k}$

We can compute the cross power spectrum $\left\langle a_{\ell, m}^{k} F_{\ell}^{k} \times a_{\ell, m}^{j} F_{\ell}^{j}\right\rangle$ and compare it to the cross power spectrum $\left\langle a_{\ell, m}^{j} F_{\ell}^{j} \times a_{\ell, m}^{W_{1}} F_{\ell}^{W_{1}}\right\rangle$ : the ratio is the relative calibration of channel $k$ against the reference channel $W_{1}$ of $\mathrm{B} 03$ :

$\mathcal{R}_{k, W_{1}}=\frac{\left\langle a_{\ell, m}^{k} F_{\ell}^{k} \times a_{\ell, m}^{j} F_{\ell}^{j}\right\rangle}{\left\langle a_{\ell, m}^{j} F_{\ell}^{j} \times a_{\ell, m}^{W_{1}} F_{\ell}^{W_{1}}\right\rangle}$.

This is iterated for all $j \neq k$ for consistency checks. This procedure needs Monte-Carlo simulations to properly take into account any correlated noise between B03 channels $i$ and $j$.

To avoid this, we can use non-B03 datasets (the $\mathrm{V}$ and $\mathrm{W}$ WMAP maps and the $150 \mathrm{GHz}$ B98 map, denoted by greek indices) as pivot signals:

$\mathcal{R}_{k, W_{1}}=\frac{\left\langle a_{\ell, m}^{k} F_{\ell}^{k} \times a_{\ell, m}^{\alpha} F_{\ell}^{\alpha}\right\rangle}{\left\langle a_{\ell, m}^{\alpha} F_{\ell}^{\alpha} \times a_{\ell, m}^{W_{1}} F_{\ell}^{W_{1}}\right\rangle}$.

To improve the accuracy, we also use slightly more complex combinations of signals:

$\mathcal{R}_{k, W_{1}}=\frac{\left\langle a_{\ell, m}^{k} F_{\ell}^{k} \times a_{\ell, m}^{\alpha} F_{\ell}^{\alpha}\right\rangle}{\left\langle a_{\ell, m}^{\alpha} F_{\ell}^{\alpha} \times a_{\ell, m}^{i} F_{\ell}^{i}\right\rangle} \cdot \frac{\left\langle a_{\ell, m}^{i} F_{\ell}^{i} \times a_{\ell, m}^{\beta} F_{\ell}^{\beta}\right\rangle}{\left\langle a_{\ell, m}^{\beta} F_{\ell}^{\beta} \times a_{\ell, m}^{W_{1}} F_{\ell}^{W_{1}}\right\rangle}$

and average all the multipole bins between $\ell=75$ and $\ell=500$. Using Eqs. (32) and (33) we have obtained 21 estimates of the relative calibration for each $\mathrm{B} 03$ channel. We have computed the weighted average of these estimates, and checked with MonteCarlo simulations that such procedure is accurate and that the standard error on the weighted average is a correct estimate of the statistical uncertainty. The results are reported in Table 8 . These relative calibrations are consistent with the pixel-space ones obtained in Sect. 7.2.1, and the statistical uncertainty is smaller, ranging between $\sim 0.2 \%$ and $\sim 0.4 \%$. We have also verified that using either of the two sets of relative calibrations does not change the combined map significantly.

Having measured the relative calibrations for all channels of the same band, we can combine them into an uncalibrated optimal map of the sky, using the optimal map-making described in Sect. 7.1.

We then estimate the absolute calibration of this map by comparing it to the WMAP map in the same region. The WMAP all-sky maps are calibrated with remarkable accuracy $(0.5 \%$, see Bennett et al. 2003). So we can compare the angular power spectra measured by B03 and WMAP in the same sky region in order to measure the absolute responsivity $\mathcal{S}$ of B03.

Assuming that the uncalibrated signal in B03 (in Volt) is related to the $\mathrm{CMB}$ temperature measured by WMAP as $T_{\mathrm{WMAP}}=$ $V_{B} / \mathcal{S}$, we can estimate $\mathcal{S}$ from different combination of B03 and WMAP power spectra. We use the following one:

$\frac{1}{\mathcal{S}}=\frac{\left\langle a_{\ell, m}^{B} F_{\ell}^{B} \times a_{\ell, m}^{\mathrm{WMAP}} F_{\ell}^{\mathrm{WMAP}}\right\rangle}{\left\langle a_{\ell, m}^{B, \mathrm{WX}} F_{\ell}^{\mathrm{WX}} \times a_{\ell, m}^{B, \mathrm{YZ}} F_{\ell}^{\mathrm{YZ}}\right\rangle}$.

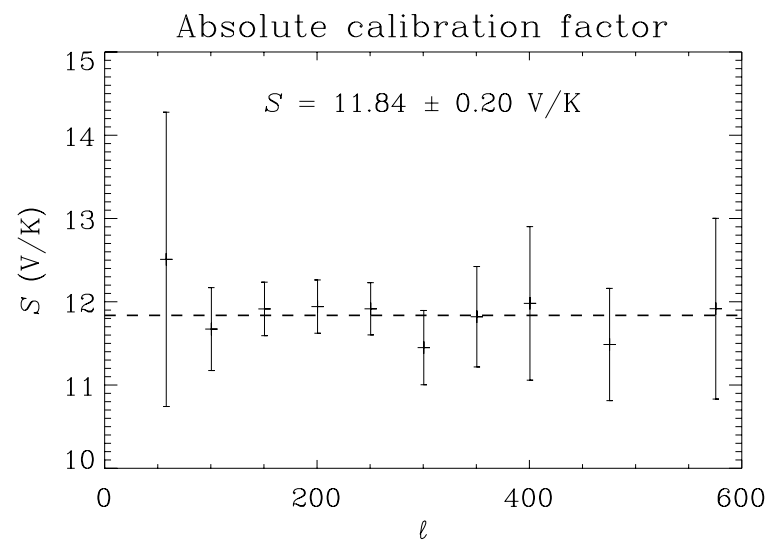

Fig. 24. Absolute calibration factors $\mathcal{S}_{\ell}$ based on WMAP measurement. The cross-power spectra are computed using the shallow integration region with uniform weight. The final value for $\mathcal{S}$ is the weighted average of the $\mathcal{S}_{\ell}$.

For the $145 \mathrm{GHz}$ PSBs, we compute the cross-power spectrum of $\left\langle a_{\ell, m}^{B} F_{\ell}^{B} \times a_{\ell, m}^{\mathrm{WMAP}} F_{\ell}^{\mathrm{WMAP}}\right\rangle$ using optimal combined maps of all channels for both the experiments, and the cross-power spectrum of $\left\langle a_{\ell, m}^{B, \mathrm{WX}} F_{\ell}^{\mathrm{WX}} \times a_{\ell, m}^{B, \mathrm{YZ}} F_{\ell}^{Y Z}\right\rangle$ using optimal maps obtained combining the $W$ and $X$ PSBs for the first map, and the $Y$ and $Z$ PSBs for the second one. Using this denominator in place of $\left\langle a_{\ell, m}^{B} F_{\ell}^{B} \times a_{\ell, m}^{B} F_{\ell}^{B}\right\rangle$ we avoid the need to remove a bias due to correlated noise. This choice, based on half of the detectors, does not degrade the precision of the result, which is already limited by WMAP noise.

For the $145 \mathrm{GHz} \mathrm{T}$ map, we show in Fig. 24 the measurement of $\mathcal{S}$ obtained from Eq. (34), i.e. the absolute calibration factor as a function of $\ell$. The error bars are estimated with a bootstrap method (see Polenta 2004 for details). Its flatness confirms that we are properly correcting for beam, pixelization and noise effects.

We confirm the robustness of the result by computing calibration factors from the shallow and deep surveys separately and by applying different weighting schemes. The final result for the $145 \mathrm{GHz}$ map is

$\mathcal{S}_{145}=(11.84 \pm 0.20) V / K$

where the 1- $\sigma$ error already incorporates the effect of the calibration uncertainty of WMAP. The absolute responsivity of a single channel $k$ can be obtained from the values listed in Table 8 as $\mathcal{S}_{k}=\mathcal{S R}_{k, W_{1}}$.

The calibrations of 245 and $345 \mathrm{GHz}$ maps are found in a similar way. We obtain

$\mathcal{S}_{245}=(4.27 \pm 0.20) \mathrm{V} / \mathrm{K}$

and

$\mathcal{S}_{345}=(3.04 \pm 0.27) \mathrm{V} / \mathrm{K}$

\subsection{Differences in the two pipelines}

Before discussing the results of the analysis, we summarize in Table 9 the most important differences between the two pipelines. Despite the many different choices, the final results of the analysis are very consistent for both the maps (see Sect. 7.4 and following) and the power spectra (Jones et al. 2006a; Piacentini et al. 2006; Montroy et al. 2006). 
Table 9. Summary of the different choices made for the two data analysis pipelines.

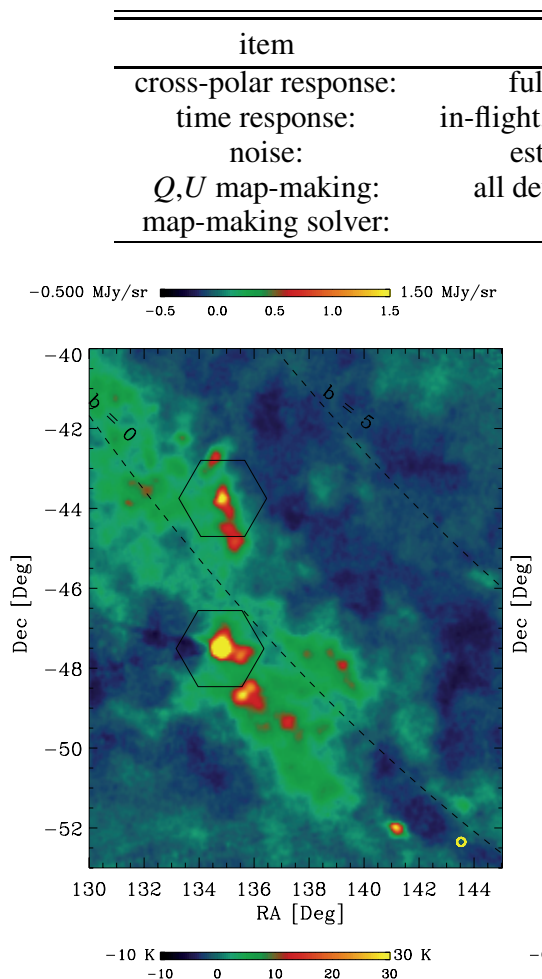

IT pipeline
still beam (Table 4, Col. 4)
stimated on the full flight
Conjectors treated individually
Conjugate Gradients

\begin{tabular}{c} 
NA pipeline \\
axial (Table 4, Col. 5) \\
m lab measurements (Sect. 3.1) \\
stimated in $\sim$ hour long chunks \\
PSB pairs differences \\
Jacobi \\
\hline
\end{tabular}
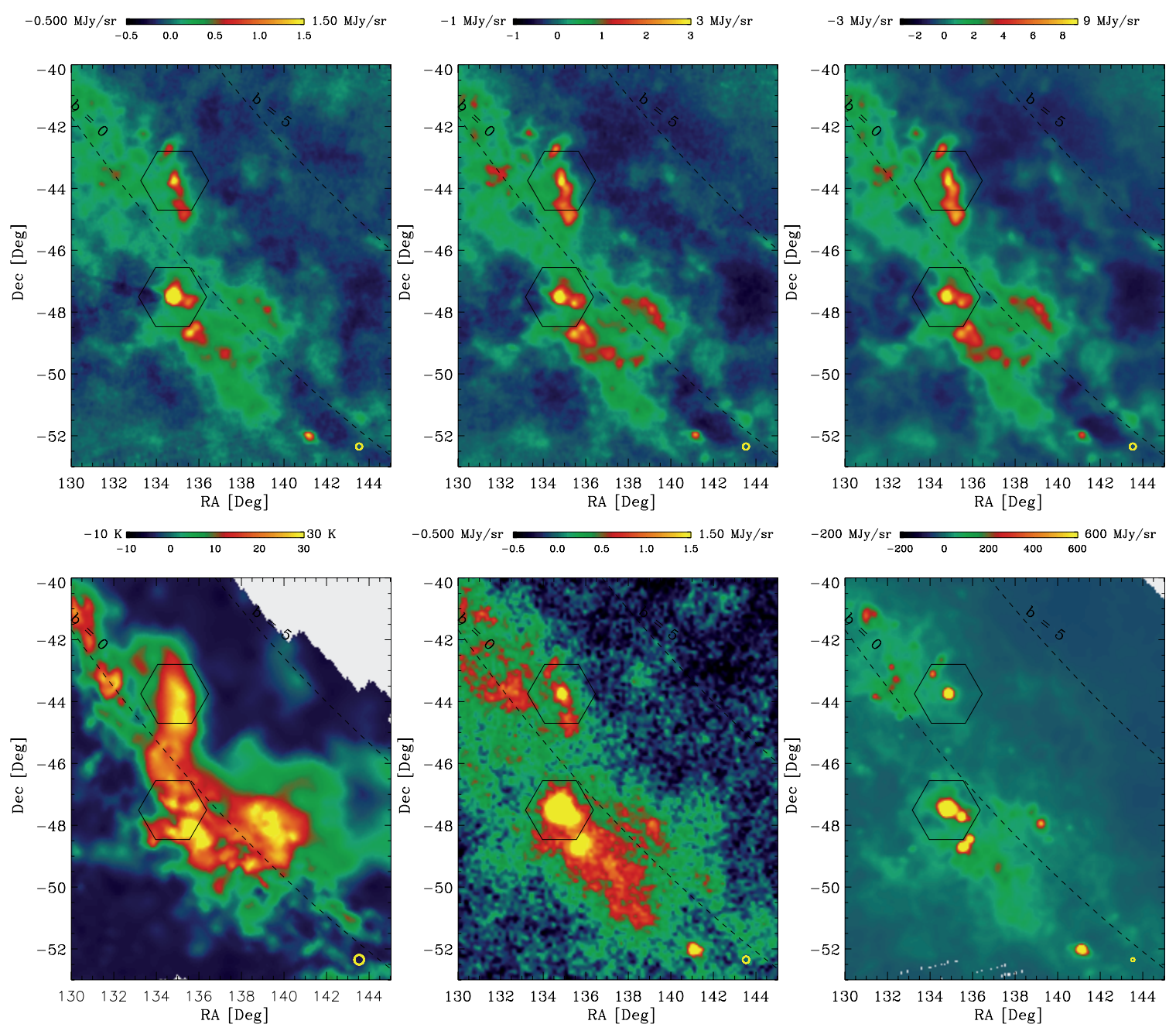

Fig. 25. в03 survey of the Galactic plane. In the top row we display (left to right): B03 maps at 145, 245 and 345 GHz. The brightness units are $\mathrm{MJy} / \mathrm{sr}$. The conversion factors from MJy/sr to $m K_{\mathrm{CMB}}$ are given in Table 3. In the bottom row we report, for comparison, maps of the same region from (left to right): the CO survey of Dame et al. (2001), the WMAP $94 \mathrm{GHz}$ channel, and the IRAS $3000 \mathrm{GHz}$ survey. All the maps have been convolved with a $6^{\prime} F W H M$ Gaussian filter. The equivalent $F W H M$ resolution of the maps is indicated by the yellow circle on the bottom right of each panel. The hexagons locate RCW38 (Dec -47.5 ) and IRAS $08576\left(\operatorname{Dec} \sim-43.8^{\circ}\right)$.

\subsection{Observations of the galactic plane}

\subsubsection{Brightness maps of the galactic plane}

Brightness maps of a section of the Galactic plane at 145, 245 and $345 \mathrm{GHz}$ are shown in Fig. 25, in comparison with other relevant maps of emission from dust and associated molecular gas. Several known HII regions are evident in the maps. Particulary luminous are $\mathrm{RCW} 38$ (at $\mathrm{RA}=134.76^{\circ}$, $\mathrm{Dec}=-47.47^{\circ}$ ) and IRAS $08576\left(\right.$ at RA $\left.=134.92^{\circ}, \mathrm{Dec}=-43.75^{\circ}\right)$. Diffuse emission is also evident. It is instructive to compare the B03 maps to monitors of different components of the ISM, like the IRAS/DIRBE maps for the dust continuum and the $115 \mathrm{GHz} \mathrm{CO}$ map of Dame et al. (2001) for the molecular gas (see bottom row of
Fig. 25). There is a good correlation between the B03 maps and the IRAS/DIRBE $3000 \mathrm{GHz}$ map for most of the diffuse structure observed. A striking exception is the cloud evident in all B03 channels at $\mathrm{RA}=135.24^{\circ}$ and $\mathrm{Dec}=-44.80^{\circ}$ : this is very dim in the IRAS map, while it is bright in the CO map and is also visible in the WMAP $94 \mathrm{GHz}$ map: the signature of a cold dust cloud associated with molecular gas.

The measured spectral flux density (SFD) of the main Galactic sources measured by B03 is reported in Fig. 26. The fluxes have been obtained integrating the brightness maps on disks centered on the sources. The diameter of the disks is chosen as the maximum between the apparent size of the source and twice the $F W H M$ of the experiment beam. The error in the 

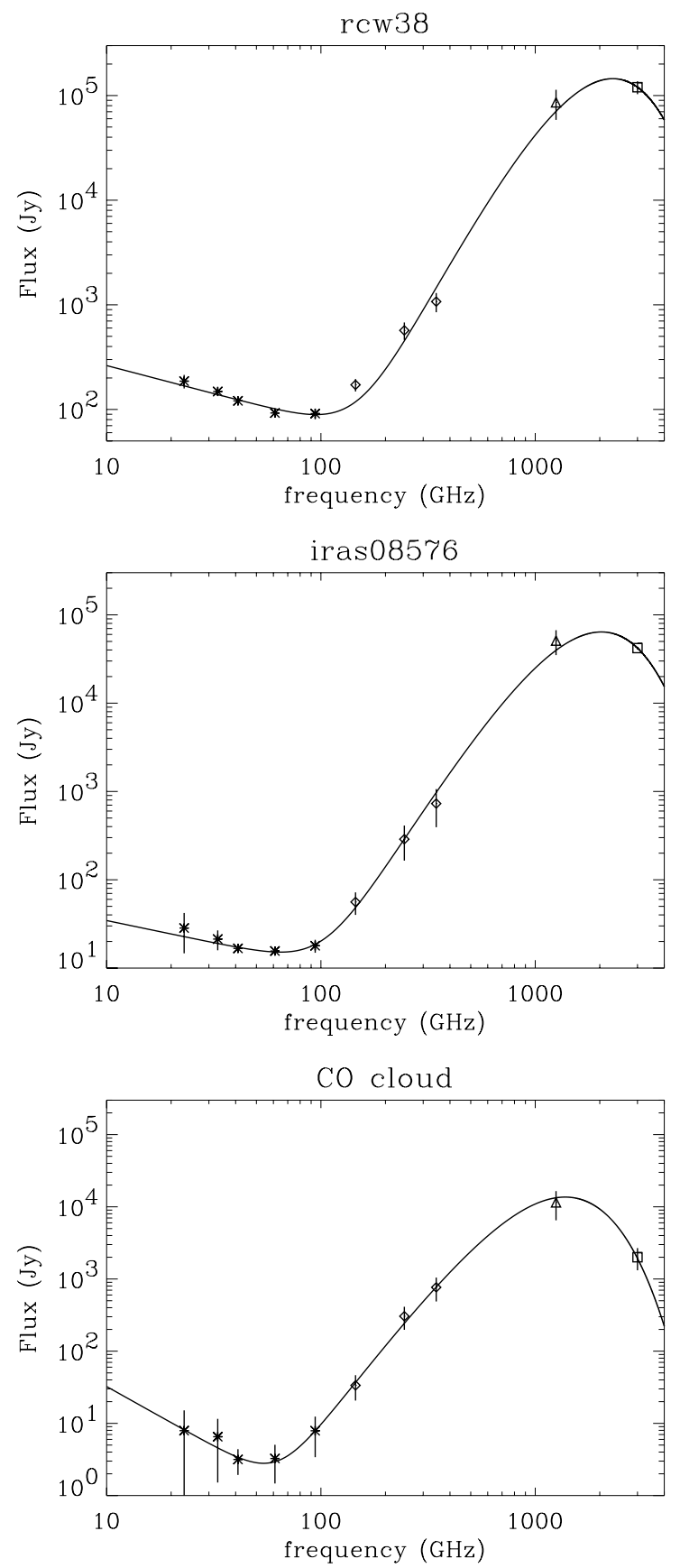

Fig. 26. Measured integrated fluxes for selected Galactic sources observed by B03 (diamonds). The other data points are from WMAP (stars), from DIRBE (triangle) and from the IRAS/DIRBE map (square). The continuous line is the best fit SFD obtained as the sum of a power law at low frequencies $\left(\sim v^{a}\right)$ plus a thermal dust spectrum dominating at high frequencies $\left(\sim v^{2} B\left(v, T_{\mathrm{d}}\right)\right)$.

determination of the flux is dominated by the presence of diffuse emission, which must be subtracted to estimate the net flux of the source: this is less critical for RCW38, which is a relatively isolated source. This results in error bars larger than those from calibration and pointing jitter.

In order to gain insight on the physical processes of emission operating in the sources, we combined the B03 SFD data with data from WMAP, DIRBE and IRAS (see Fig. 26). A combination of a power law dominating at low frequencies $\left(\sim v^{a}\right)$, and a dust-spectrum at high frequencies $\left(\sim v^{2} B\left(v, T_{\mathrm{d}}\right)\right)$ produces a good fit to the data.
We find dust temperatures of $(22.4 \pm 0.9) \mathrm{K},(19.6 \pm 0.7) \mathrm{K}$, and $(13.3 \pm 0.6) \mathrm{K}$ for RCW38, IRAS 08576, and the CO cloud respectively. The spectral index of the power law is $(-0.5 \pm 0.1)$, $(-0.5 \pm 0.3)$, and $(-1.7 \pm 1.3)$ for RCW38, IRAS 08576, and the CO cloud respectively.

A word of caution is necessary for the power-lax indices derived here. We are using data from experiments with different angular resolution. In particular the low frequency data have poorer angular resolution, so the measured flux can be contaminated by nearby sources entering these wider beams. For this reason the spectra shown in Fig. 26 could be increasingly contaminated (biased high) at low frequencies. This issue will be analyzed in a future publication.

The S/N ratio of the observations of RCW38 is high enough that we can also give an upper limit for the ellipticity of our beam. We fitted the $145 \mathrm{GHz}$ map of RCW38 with an elliptic Gaussian, leaving as free parameters the two FWHMs, the orientation angle, and the amplitude. The beam FWHMs we find are consistent with the ones discussed in Sect. 6.2. The measured ellipticity is $(4 \pm 4) \%$. This includes the effects of beam ellipticity, anisotropic pointing jitter and intrinsic source ellipticity. The cross-link of the sky scans (see Sect. 2.2) is such that the effect of an elliptic beam could not cancel out. It is thus unlikely that the beam ellipticity is $>10 \%$; according to the analysis carried out in Sect. 2.6, the contamination of our polarization measurements due to beam ellipticity is thus expected to be less than a few $\%$.

\subsubsection{Polarization of RCW38}

RCW38 is the brightest source we observed. At $145 \mathrm{GHz}$ the polarization of RCW38 is very low. We have carried out an analysis on the $145 \mathrm{GHz} \mathrm{W}, \mathrm{X}$, and Y PSBs, analyzing 53 scans over the source, and assuming that there is a constant polarization over the size of the source. For each scan and for each of the 6 bolometers we fit a maximum amplitude of the detected signal. We then solve for $Q$ and $U$ of each scan using the detector signal differences W1-W2, X1-X2, Y1-Y2, taking into account the relative calibration of the bolometers, the color corrections, and the orientation angles. The resulting average values for $Q$ and $U$ are $Q_{\mathrm{RCW} 38}=(-0.046 \pm 0.076) \mathrm{MJy} / \mathrm{sr}$ and $U_{\mathrm{RCW} 38}=(0.054 \pm 0.082) \mathrm{MJy} / \mathrm{sr}$. Since the average brightness for RCW38 on the same scans is $3.7 \mathrm{MJy} / \mathrm{sr}$, the upper limit for the polarization degree is $p_{\mathrm{RCW} 38}<6 \%$ (2- $\sigma$ U.L.).

\subsection{Brightness maps at high galactic latitude}

\subsubsection{Maps of CMB temperature anisotropy at high galactic latitude}

The main product of this experiment is the $145 \mathrm{GHz}$ map, which is shown as a large image in Fig. 27. The structure visible in the map with high $\mathrm{S} / \mathrm{N}$ is $\mathrm{CMB}$ anisotropy. ISD is negligible in comparison to the CMB, as obtained in B98 (Masi et al. 2001) and as we confirm below with the B03 data.

Maps of the shallow and deep regions at 145, 245 and $345 \mathrm{GHz}$ are compared in Figs. 28 and 29 respectively. For a meaningful comparison, all maps have been filtered in the same way. Aggressive high-pass filtering of the time ordered data (cuton at 7.5 times the scan frequency) was required for the $245 \mathrm{GHz}$ and $345 \mathrm{GHz}$ bolometers, in order to avoid artifacts due to scan synchronous noise. The brightness at 245 and 345 is due to a mixture of $\mathrm{CMB}$ and emission from interstellar dust (ISD), 


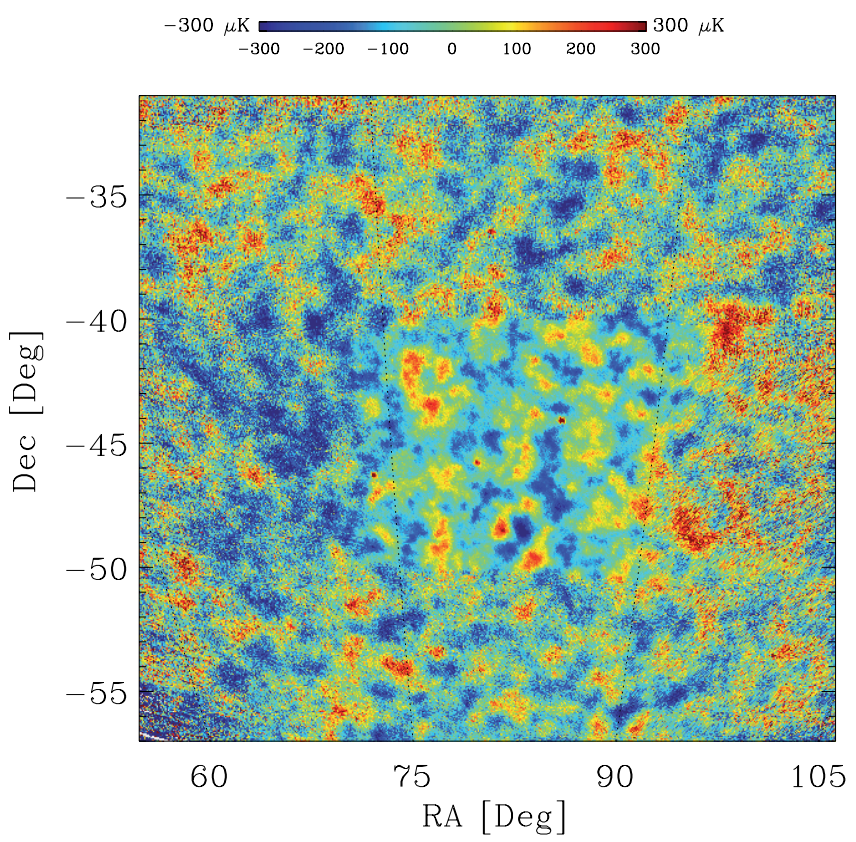

Fig. 27. $145 \mathrm{GHz} I$ map from all the PSB bolometers of B03. $I$ is encoded in the false color scale in thermodynamic temperature units for a $2.725 \mathrm{~K}$ blackbody. The pixel size is 3.4 arcmin. The data of this map will be made publicly available (together with a set of realistic simulations needed for quantitative analysis) at the B03 web servers: http://oberon.roma1.infn.it/boomerang/b2k and http: //cmb.phys. case.edu/boomerang.

as evident from the comparison of the CMB-subtracted maps to the IRAS $3000 \mathrm{GHz}$ map in the botton rows of Fig. 28.

The temperature anisotropy of the $\mathrm{CMB}$ is measured with high $\mathrm{S} / \mathrm{N}$ in the deep $145 \mathrm{GHz}$ map. Figure 30 compares the $145 \mathrm{GHz}$ deep map with maps of the same region obtained from BOOMERanG-98 at $150 \mathrm{GHz}$, and from WMAP at at $94 \mathrm{GHz}$. There is excellent morphological agreement between all three maps.

To estimate more precisely the $\mathrm{S} / \mathrm{N}$ of the $145 \mathrm{GHz}$ deep map, we jackknife the data to provide two independent maps: one using data only from the first half of the deep survey (D1) and one using data only from the second half of the deep survey (D2). We then form the sum and difference maps, $(\mathrm{D} 1+\mathrm{D} 2) / 2$ and (D1-D2)/2. The sum map contains both signal and noise, the difference map only noise. For all maps we have used HEALPIX NSIDE $=1024$, i.e. a pixel side of $3.5^{\prime}$. Histograms of both maps, shown in Fig. 31, are Gaussian-distributed. The rms in the sum and difference maps is $\sigma_{+}=(93.2 \pm 0.5) \mu \mathrm{K}$ and $\sigma_{-}=(23.4 \pm 0.2) \mu \mathrm{K}$, respectively. The rms of the sky signal is thus $\sqrt{93.2^{2}-23.4^{2}}=(90.2 \pm 2.3) \mu \mathrm{K}$, where the error now includes the uncertainty in absolute calibration. Realistic simulations of our observations, including all the details of scan speed, coverage, detectors noise, filtering, pixelization, etc. can be used to estimate the expected rms of CMB anisotropy in these observations, for the standard "concordance" model best fitting WMAP. We used the same simulations used for our spectral analysis (Jones et al. 2006a; Piacentini et al. 2006; Montroy et al. 2006). The result is $(94 \pm 13) \mu \mathrm{K}$ (including cosmic variance). A similar result is obtained integrating the power spectrum of the "concordance" model over the B03 window function shown in Fig. 16.

The distribution of the measured brightness in the deep $145 \mathrm{GHz}$ map is accurately Gaussian. The simplest Gaussianity test is the evaluation of the skewness $S_{3}$ and kurtosis $S_{4}$ of the pixel temperature distribution, computed as

$$
S_{3}=\frac{1}{N} \sum_{i=1}^{N}\left[\frac{T_{i}-\langle T\rangle}{\sigma_{T}}\right]^{3} ; \quad S_{4}=\frac{1}{N} \sum_{i=1}^{N}\left[\frac{T_{i}-\langle T\rangle}{\sigma_{T}}\right]^{4}-3
$$

In Fig. 32 we compare the measured skewness $\left(S_{3}=-0.062\right)$ and kurtosis $\left(S_{4}=-0.053\right)$ to the distribution of $S_{3}$ and $S_{4}$ evaluated from our realistic simulations set. The agreement is very good: no hints for any deviation from Gaussianity. This also confirms that systematic effects and foreground contamination are negligible in this map.

The CMB structures mapped at $145 \mathrm{GHz}$ are evident also in the 245 and $345 \mathrm{GHz}$ maps (Fig. 29), even if with lower S/N, due to the higher background on the bolometers, to the lower number of detectors in these bands, and to the presence of increasing contamination from interstellar dust emission.

\subsubsection{Foreground contamination by diffuse ISD emission at high latitude}

Our multiband maps allow us to carry out basic tests on the level of foreground contamination. We consider first the deep survey (carried out at Galactic latitudes $|b|>25^{\circ}$ ). We use the SFD IRAS map $D_{i, j}$ at $100 \mu \mathrm{m}$, corrected using DIRBE data (Schlegel et al. 1999), as a template for ISD emission. We use the WMAP $94 \mathrm{GHz}$ map $W_{i, j}$ as a template for CMB anisotropy. Both maps have been sampled along the scans of the B03, and then high-pass and low-pass filtered using the transfer function of the $145 \mathrm{GHz}$ B03 detectors, in order to create the corresponding synthesized time-streams. These have been processed in the same way as the B03 detectors (see Sect. 7.5.1), to obtain the maps shown in Figs. 28, 29 and 30. For this particular analysis we use a $14^{\prime}$ pixelization, in order to avoid problems with the different beam size of the instruments, and carry out a linear fit in pixel space:

$I_{i, j}=A W_{i, j}+B D_{i, j}+C$

where $I_{i, j}$ is one of the three B03 maps $(145,245,345 \mathrm{GHz})$. The results of the fits are reported in Table 10. The errors have been estimated by dividing the deep survey region into nine subregions, performing the fit for each subregion, and computing the standard deviation of the best fit parameters. In this way we account for variations in the properties of the foreground over the survey region. The $A$ values are not reported since they are biased low, due to the relatively high noise of the WMAP map. Simulations show that, with the noise levels present here, this problem does not bias the estimates of the $B$ values by more than a few $\%$. This error is added in quadrature to the statistical error, which dominates the total error.

The fact that the correlation coefficient for the WMAP template is always significant means that we have detected CMB anisotropy in all of our channels. The correlation coefficient for the IRAS template, instead, is statistically significant for the 245 and for the $345 \mathrm{GHz}$ survey. This means that the best fit values for $B$ at $145 \mathrm{GHz}$ provide only an upper limit for ISD contamination. As an example of a region where ISD fluctuations are larger, we can consider the part of the Shallow Survey closer to the Galactic Plane: $90^{\circ}<\mathrm{RA}<105^{\circ} ;-50^{\circ}<$ Dec $<-40^{\circ}$. Proceeding as before, in this region we obtain the results reported in the lower part of Table 10, which confirm the results found for the deep survey.

We have carried out an analysis similar to the one just described, using the difference maps $M_{345}=I_{345}-I_{145}$ and 

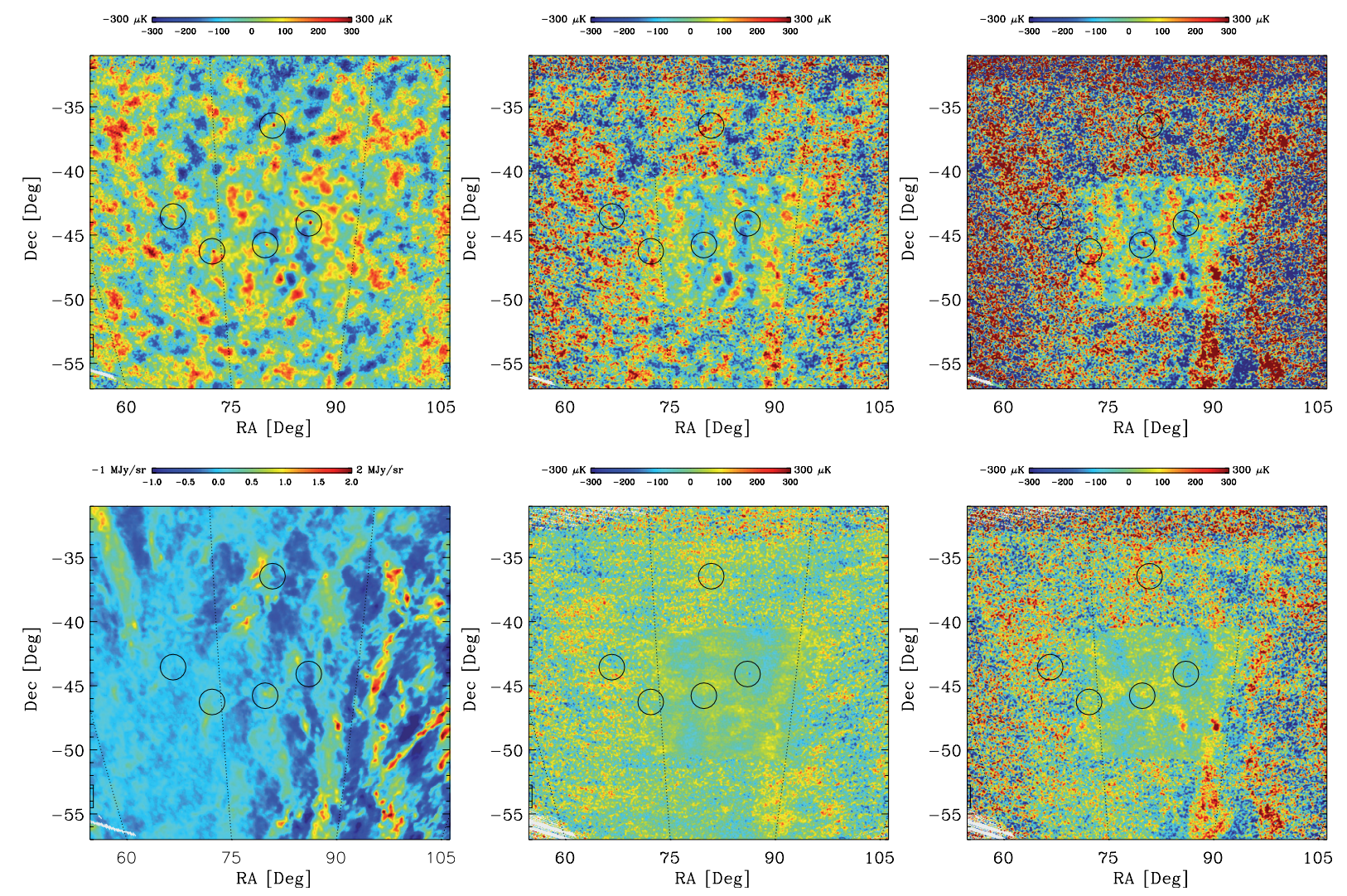

Fig. 28. The shallow and deep surveys of B03. Top row: I maps from B03. From left to right: $145 \mathrm{GHz} ; 245 \mathrm{GHz} ; 345 \mathrm{GHz}$. I is encoded in the false color scale in thermodynamic temperature units for a $2.725 \mathrm{~K}$ blackbody. The pixel size is 3.4 arcmin. Bottom row, from left to right: IRAS/DIRBE image of the same region at $3000 \mathrm{GHz}$; B03 difference map obtained by subtracting the $145 \mathrm{GHz}$ map from the $245 \mathrm{GHz}$ map, in order to remove CMB anisotropy from the resulting map; B03 difference map obtained as $345 \mathrm{GHz}$ map minus $145 \mathrm{GHz}$ map. The central region, where the noise is evidently lower, is the deep survey. In Fig. 29 we zoom on such region. The circles label the positions of known AGNs. To remove large-scale gradients, a high-pass filter at 7.5 times the scan frequency was applied on the time-ordered data: this filter is needed to remove artifacts due to scan synchronous noise in the 245 and $345 \mathrm{GHz}$ maps. A Gaussian filter with 7' FWHM was used as anti-aliasing.

$M_{245}=I_{245}-I_{145}$ as "CMB-subtracted" maps. Since our $145 \mathrm{GHz}$ map is dominated by CMB anisotropy and features very low noise, $M_{345}$ and $M_{245}$ monitor all non-CMB brightness, and have lower noise than what we can obtain subtracting the $94 \mathrm{GHz}$ WMAP map as a CMB template. The fit of equation

$M_{i, j}=B D_{i, j}+C$

gives results very similar to the fits to Eq. (39) reported in Table 10.

The rms fluctuation of the dust template in the deep survey region is about $0.25 \mathrm{MJy} / \mathrm{sr}$, so we get $\Delta T_{\text {dust,rms }}=(12 \pm$ 5) $\mu \mathrm{K}_{\mathrm{CMB}}$ at $245 \mathrm{GHz}$ and $\Delta T_{\text {dust,rms }}=(50 \pm 5) \mu \mathrm{K}_{\mathrm{CMB}}$ at $345 \mathrm{GHz}$. The upper limit at $145 \mathrm{GHz}$ is $\Delta T_{\text {dust,rms }}<10 \mu \mathrm{K}_{\mathrm{CMB}}$ (1- $\sigma$ u.1.).

Finkbeiner et al. (1999) have studied the extrapolation to longer wavelengths of dust fluctuations detected by IRAS. Our results are consistent with their extrapolations. Their model 8 for the specific brightness of ISD is:

$B_{\text {dust }}(v) \propto q f_{1} v^{\alpha_{1}} B\left(v, T_{1}\right)+\left(1-f_{1}\right) v^{\alpha_{2}} B\left(v, T_{2}\right)$

with $\alpha_{1}=1.67, T_{1}=9.4 \mathrm{~K}, \alpha_{2}=2.7, T_{2}=16.2 \mathrm{~K}, f_{1}=0.0363$, $q=13$. The rms of the extrapolated maps in the deep survey region are 2,11 and $47 \mu \mathrm{K}_{\mathrm{CMB}}$ in the 145,245 and $345 \mathrm{GHz}$ bands respectively. So this model fits quite well our measurements (see also Fig. 33).

With no detection at $145 \mathrm{GHz}$, we use the spectrum of Model 8 to extrapolate - with all the necessary caveats - to
$145 \mathrm{GHz}$. Integrating in our bands (specified in Fig. 10), and normalizing to our $345 \mathrm{GHz}$ point, we get $\Delta T_{\text {dust,rms }}=(2.9 \pm$ 1.2) $\mu \mathrm{K}_{\mathrm{CMB}}$ at $145 \mathrm{GHz}$. This value is much smaller than the CMB anisotropy at the same frequency (the total sky rms at $145 \mathrm{GHz}$ in our window function is $\sim 90 \mu \mathrm{K}$ ).

The dust fluctuations are non-Gaussian. However, only very few structures deviate significantly from the rms stated above: these are the clouds visible in the 354-145 difference map at $(\mathrm{RA}, \mathrm{Dec})=\left(88.5^{\circ},-48.3^{\circ}\right)$ and $\left(84.6^{\circ},-48.2^{\circ}\right)$. Their size is $\lesssim 1^{\circ}$, and their brightness, extrapolated at $145 \mathrm{GHz}$ with Model 8 , is $\lesssim 20 \mu \mathrm{K}$.

Since the polarization of the diffuse cirrus at these frequencies is $\lesssim 10 \%$ of the brightness (Ponthieu et al. 2005), we expect a polarized contribution from ISD $\lesssim 1 \mu \mathrm{K} \mathrm{rms,} \mathrm{which} \mathrm{is} \mathrm{small}$ with respect to the expected CMB polarization $(\sqrt{\langle E E\rangle} \sim 3 \mu \mathrm{K}$ rms in our beam and with $14^{\prime}$ pixelization we are using for the analysis in this paragraph).

The contamination estimates above depend on the assumption that ISD is well monitored by the IRAS/DIRBE template. They do not take into account the possible existence of a colder dust component, undetected by IRAS, and with a different angular distribution.

Studying the residuals from the best fit of Eqs. (39) and (40), we find that the $145 \mathrm{GHz}$ residuals are consistent with instrumental noise, while there are, in the 245 and $345 \mathrm{GHz}$ residuals, structures which could be either instrumental artifacts or real sky fluctuations, or a combination of the two. The SFD extrapolation 

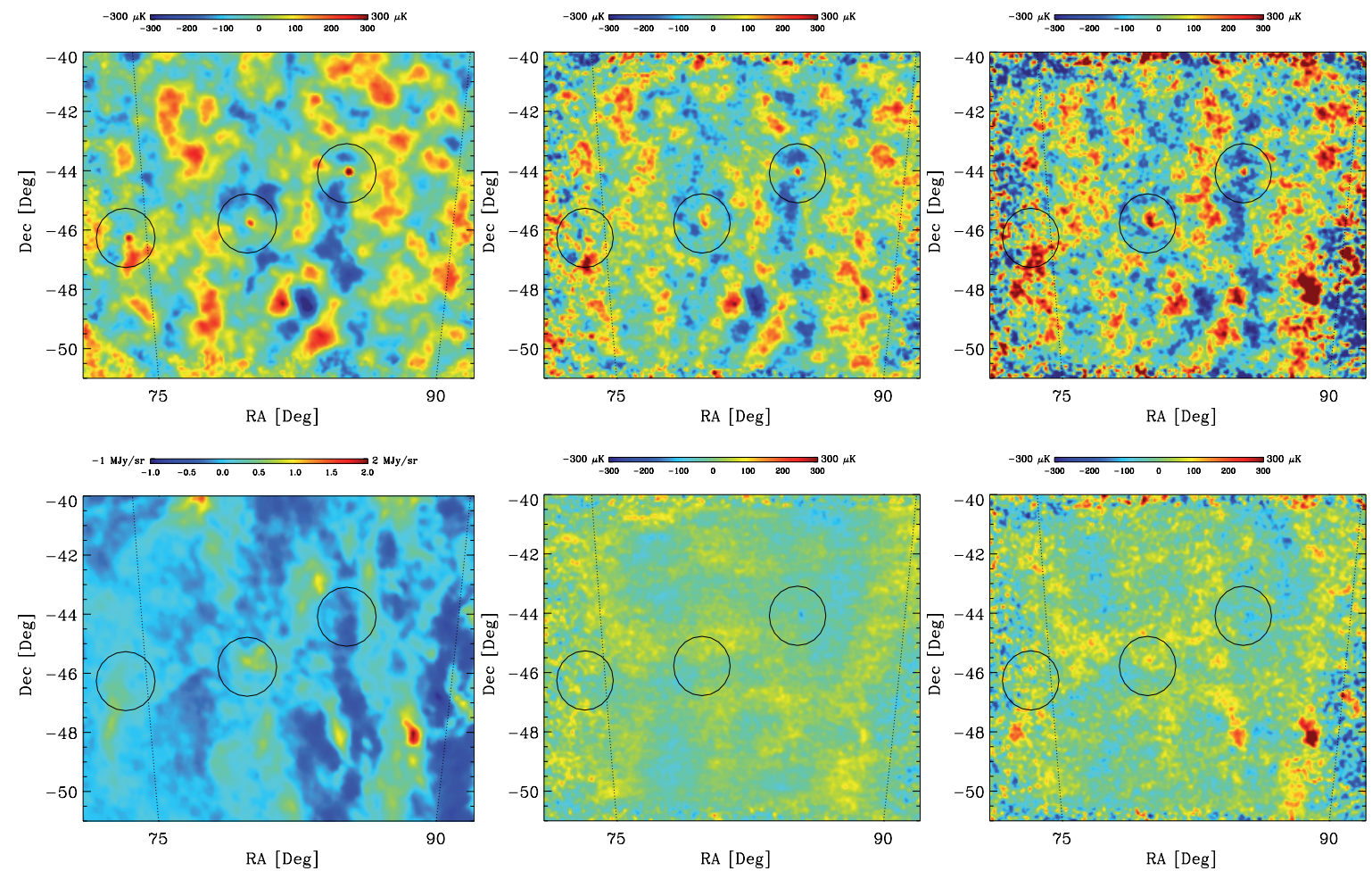

Fig. 29. The deep survey of в03. Top row: I maps from B 03 . From left to right: $145 \mathrm{GHz} ; 245 \mathrm{GHz} ; 345 \mathrm{GHz}$. I is encoded in the false color scale in thermodynamic temperature units for a $2.725 \mathrm{~K}$ blackbody. The pixel size is 3.4 arcmin. Bottom row, from left to right: IRAS/DIRBE image of the same region at $3000 \mathrm{GHz}$; 03 difference map obtained by subtracting the $145 \mathrm{GHz}$ map from the $245 \mathrm{GHz}$ map, in order to remove CMB anisotropy from the resulting map; B03 difference map obtained as $345 \mathrm{GHz}$ map minus $145 \mathrm{GHz}$ map. All the maps have been filtered in the same way. To remove large-scale gradients, a high-pass filter at 7.5 times the scan frequency was applied on the time-ordered data: this filter is needed to remove artifacts due to scan synchronous noise in the 245 and $345 \mathrm{GHz}$ maps. A Gaussian filter with $7^{\prime} F W H M$ was used as anti-aliasing.
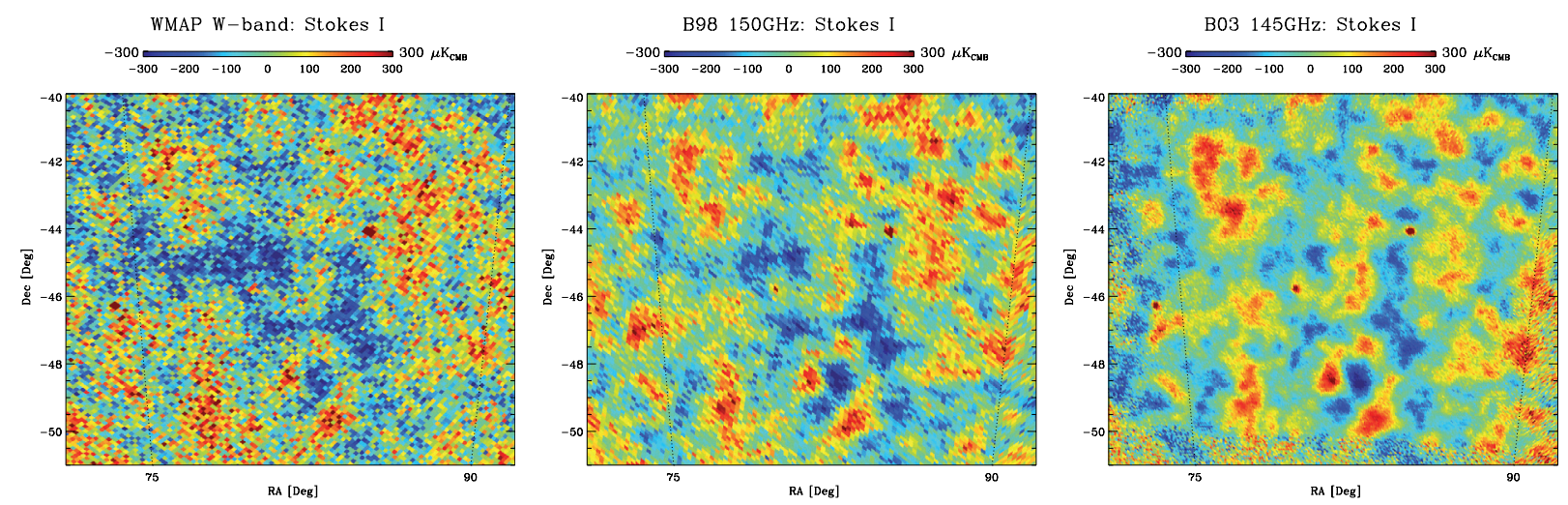

Fig. 30. Comparison of maps of the deep survey region from WMAP $94 \mathrm{GHz}$ (3-years data, left), the BOOMERanG98 data at $145 \mathrm{GHz}$ (center) and the B03 $145 \mathrm{GHz}$ data (right). The first two maps have $7^{\prime}$ pixels, the B03 one has 3.5' pixels. The improvement in the S/N of B03 is evident. A remarkable morphology agreement of the structures detected in three independent experiments is also evident. In all maps $I$ is encoded in the false colors scale in thermodynamic temperature units for a $2.725 \mathrm{~K}$ blackbody.

to our frequencies fails to produce these features (the SFD dust temperature is in fact fairly constant, at $17-18 \mathrm{~K}$, throughout the deep survey region). These structures are quite dim: the rms is $\sim 90 \mu \mathrm{K}$ at $345 \mathrm{GHz}$ and $\sim 70 \mu \mathrm{K}$ at $245 \mathrm{GHz}$. These numbers include the effect of instrumental noise and should be considered conservative upper limits for the sky fluctuations. Their extrapolation to $145 \mathrm{GHz}$, using a reasonable dust spectrum like Model 8, gives $\Delta T_{\mathrm{rms}} \lesssim 10 \mu \mathrm{K}_{\mathrm{CMB}}$ at $145 \mathrm{GHz}$.

Our deep survey region is representative of a fairly large fraction of the high latitude sky. To see this, we select increasing fractions of the sky by requiring that dust brightness, monitored by the IRAS/DIRBE map at $100 \mu \mathrm{m}$, is equal or lower than a give threshold. We find that the best $\sim 40 \%$ of the sky has the same rms fluctuation of dust brightness as in our deep survey, and that the best $75 \%$ of the sky has a brightness fluctuation $\$ 3$ times larger than the one in our deep survey.

\subsubsection{Foreground contamination by compact sources}

A few point sources are evident in the $I$ maps. These AGNs have been used for testing the pointing reconstruction procedures as explained in Sect. 5.1. The effect of the full population of resolved and unresolved AGNs as a contaminant in CMB anisotropy measurements is an important topic of discussion, 


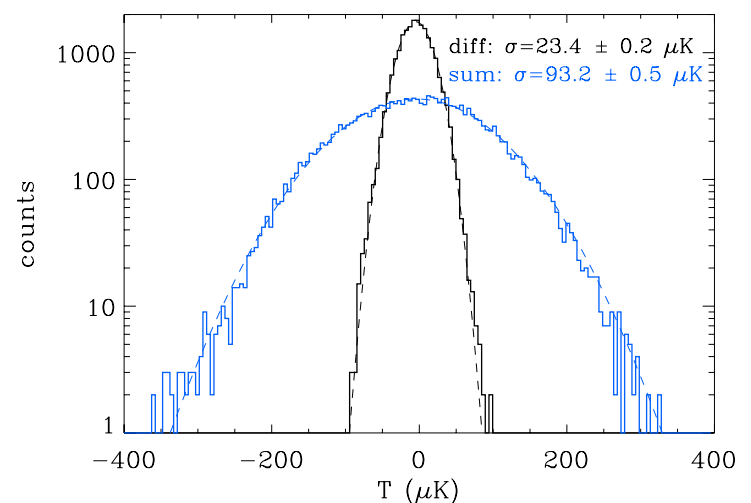

Fig. 31. Comparison of the $145 \mathrm{GHz}$ CMB I maps obtained from the two halves of the deep survey. The pixelization of the map is $3.5 \mathrm{arcmin}$ (HEALPIX NSIDE $=1024)$. We histogram pixel temperatures resulting from the difference and sum maps. Only noise and systematic effects contribute to the former, while both signal and noise contribute to the latter. The two curves are labelled with their standard deviations. The standard deviation of the signal is thus $\sqrt{93.2^{2}-23.4^{2}}=90.2 \mu \mathrm{K}$.

Table 10. Pixel-space fits of the B03 maps with a combination of a CMB template (from WMAP) and a dust template (from IRAS), see Eq. (39). For the three upper rows, the fit has been performed in the deep survey region $\left(74^{\circ}<\mathrm{RA}<90^{\circ} ;-50^{\circ}<\right.$ Dec $<-40^{\circ},\langle|b|\rangle \sim 32^{\circ}$, for a total of $217215^{\prime}$ pixels). In this region, the rms fluctuation of the IRAS/DIRBE map is $0.25 \mathrm{MJy} / \mathrm{sr}$. For the three lower rows, the fit has been done in the part of the Shallow Survey closer to the Galactic Plane $\left(90^{\circ}<\right.$ RA $<105^{\circ} ;-50^{\circ}<$ Dec $<-40^{\circ},\langle|b|\rangle \sim 23^{\circ}, 2027$ pixels of $\left.15^{\prime}\right)$. Here the rms fluctuation of the IRAS/DIRBE map is $0.57 \mathrm{MJy} / \mathrm{sr} . R(A)$ is the correlation coefficient for the CMB template, while $R(B)$ is the correlation coefficient for the dust template. The last column gives the estimated brightness fluctuation due to the ISD component correlated to the IRAS/DIRBE map, in CMB temperature units.

\begin{tabular}{ccccc}
\hline \hline Frequency & $R(A)$ & $R(B)$ & $B[\mu \mathrm{K} /(\mathrm{MJy} / \mathrm{sr})]$ & $\left\langle\Delta T_{\text {dust }}^{2}\right\rangle^{1 / 2}(\mu \mathrm{K})$ \\
\hline $145 \mathrm{GHz}$ & 0.634 & 0.074 & $(18 \pm 23)$ & $<10$ \\
$245 \mathrm{GHz}$ & 0.452 & 0.173 & $(49 \pm 21)$ & $12 \pm 5$ \\
$345 \mathrm{GHz}$ & 0.331 & 0.452 & $(202 \pm 18)$ & $50 \pm 5$ \\
\hline $145 \mathrm{GHz}$ & 0.588 & -0.085 & $(-5 \pm 9)$ & $<5$ \\
$245 \mathrm{GHz}$ & 0.273 & 0.143 & $(37 \pm 11)$ & $21 \pm 6$ \\
$345 \mathrm{GHz}$ & 0.123 & 0.380 & $(183 \pm 24)$ & $104 \pm 14$ \\
\hline
\end{tabular}

in view of the forecasted ultra-sensitive surveys of the CMB anisotropy and polarization. The SED of Blazars is almost flat $\left(S(v) \sim v^{-a}\right.$ with $0 \lesssim a \lesssim 0.3$ between few $\mathrm{GHz}$ and $\left.100 \mathrm{GHz}\right)$. At our three frequencies, bracketing the frequency of maximum brightness of CMB fluctuations, the contamination of the AGNs is thus expected to be minimal.

Giommi \& Colafrancesco (2004) have searched AGN cata$\log$ f for all the sources in the region observed by BOOMERanG. From their list of 54 sources, we find 8 AGNs in the deep survey region. Once we exclude the three brighter ones (which are evident in the map) their equivalent CMB temperature in a 9.5' beam is $<400 \mu \mathrm{K}$ at $145 \mathrm{GHz}$. Since the same region is covered by about 5000 independent beams, it is evident that the presence of mm AGN emission cannot contaminate the morphology of the $I$ maps. The integrated flux $\langle S\rangle$ and its fluctuations $\left\langle(\Delta S)^{2}\right\rangle$ produced by all resolved and unresolved AGNs with differential $\log N-\log S$ distribution $\frac{\mathrm{d} N}{\mathrm{~d} S}$ are given by

$\langle S\rangle=\int_{S_{\min }}^{S_{\max }} S \frac{\mathrm{d} N}{\mathrm{~d} S} \mathrm{~d} S$
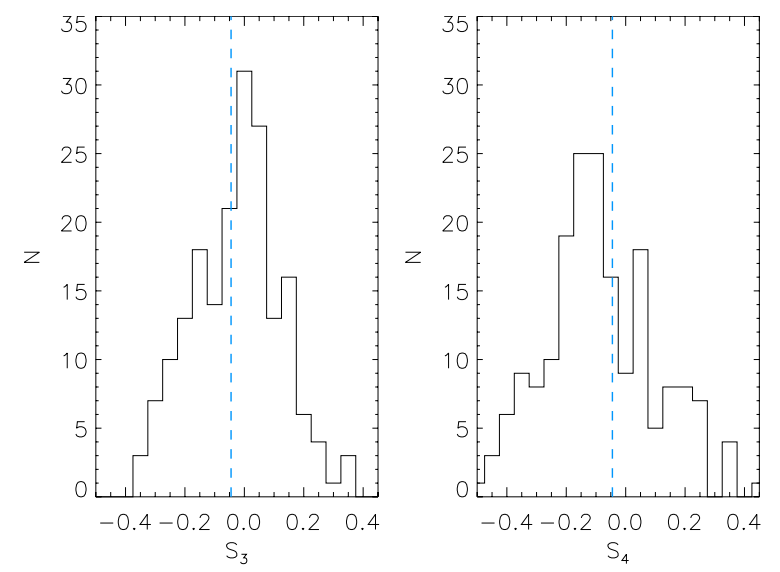

Fig. 32. The vertical lines represent the measured skewness (left panel) and kurtosis (right panel) of the pixel temperature distribution in the $145 \mathrm{GHz}$ deep survey map. The histograms derive from realistic simulations of the measurement, assuming an underlying (Gaussian) "concordance" model.

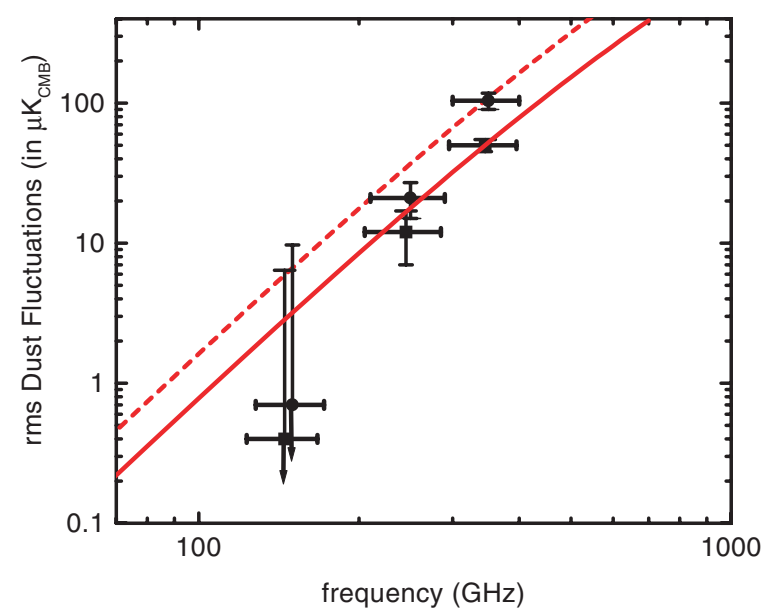

Fig. 33. Data Points: IRAS-correlated dust fluctuations detected by B03: the squares refer to the Deep Survey region $\left(74^{\circ}<\mathrm{RA}<90^{\circ}\right.$; $-50^{\circ}<$ Dec $\left.<-40^{\circ}\right)$; the circles refer to the part of the Shallow Survey closer to the Galactic Plane $\left(90^{\circ}<\mathrm{RA}<105^{\circ} ;-50^{\circ}<\right.$ Dec $<-40^{\circ}$, 2027 pixels of $15^{\prime}$ ). The continuous line is model 8 of Finkbeiner et al. (1999), normalized at $345 \mathrm{GHz}$ for the Deep Region. The dashed line is the same model, scaled to the shallow region via the ratio between the IRAS/DIRBE rms in the two regions.

$\left\langle(\Delta S)^{2}\right\rangle=\int_{S_{\min }}^{S_{\max }} S^{2} \frac{\mathrm{d} N}{\mathrm{~d} S} \mathrm{~d} S$.

When detected with an instrument with effective solid angle $\Omega$, these flux fluctuations produce a rms signal equivalent to a CMB anisotropy $\Delta T_{\mathrm{AGN}}$ given by

$\left\langle\left(\Delta T_{\mathrm{AGN}}\right)^{2}\right\rangle=T_{\mathrm{CMB}}^{2} \frac{\left\langle(\Delta S)^{2}\right\rangle}{\Omega\left[\frac{x \mathrm{e}^{x}}{\mathrm{e}^{x}-1} B\left(v, T_{\mathrm{CMB}}\right)\right]^{2}}$.

Using the $\frac{\mathrm{d} N}{\mathrm{~d} S}$ and the SED of Giommi \& Colafrancesco (2004), we get $\left\langle(\Delta S)^{2}\right\rangle \sim 30,25,21 \mathrm{Jy}^{2} / \mathrm{sr}$ at $145,245,345 \mathrm{GHz}$ respectively. Using the $\mathrm{B} 03$ beams to compute $\Omega$, we obtain $\sqrt{\left\langle\left(\Delta T_{\mathrm{AGN}}\right)^{2}\right\rangle}=5.8,4.2,6.0 \mu \mathrm{K} \mathrm{rms}$ at $145,245,345 \mathrm{GHz}$ respectively. This is negligible with respect to the measured anisotropy of the map. 

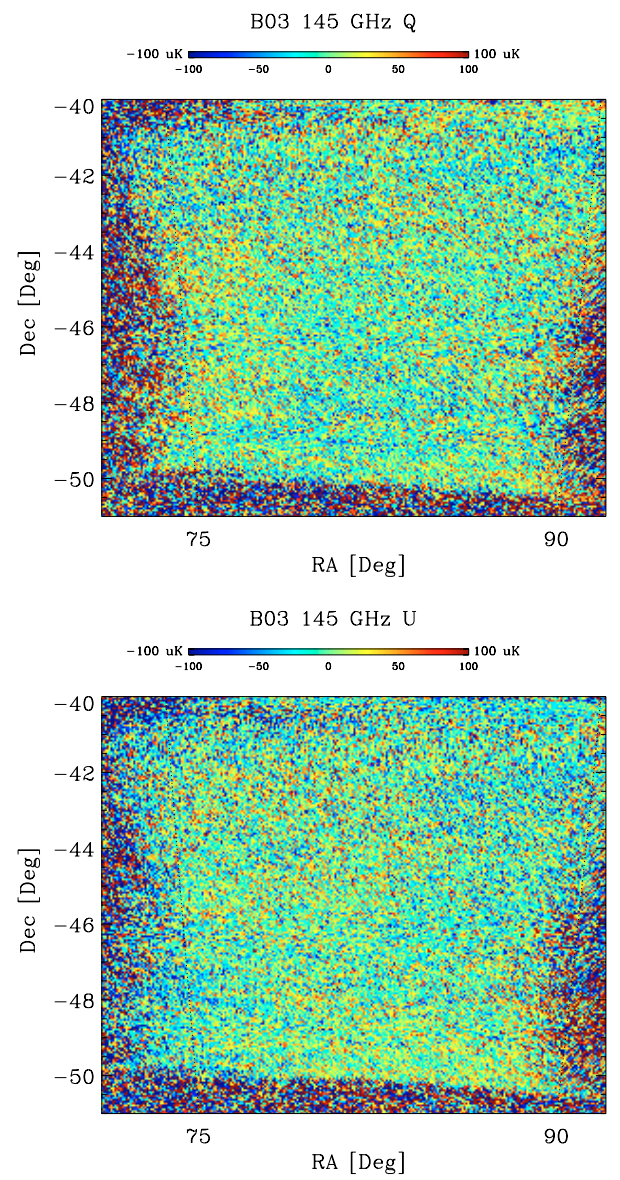

Fig. 34. Polarization in the deep survey. Top: $Q$ map from the $145 \mathrm{GHz}$ PSB bolometers; Bottom: $U$ map from the same bolometers. Note that the stretch of the false color scale is a third of the one in the T maps. These maps are dominated by instrumental noise.

AGN emission is usually polarized at a level $p \lesssim 5 \%$. Assuming random polarization directions and neglecting the natural dispersion in the $p$ values, we get

$\left\langle\left(\Delta Q_{\mathrm{AGN}}\right)^{2}\right\rangle=\frac{p^{2}}{2}\left\langle\left(\Delta T_{\mathrm{AGN}}\right)^{2}\right\rangle$.

So we find $\sqrt{\left\langle\left(\Delta Q_{\mathrm{AGN}}\right)^{2}\right\rangle}=0.20,0.15,0.21 \mu \mathrm{K} \mathrm{rms}$ at 145,245 , $345 \mathrm{GHz}$ respectively.

This is negligible with respect to the amplitude of the E-mode CMB polarization that we expect in our maps $(\sim 3 \mu \mathrm{K}$ rms for the $14^{\prime}$ pixelization, and $\sim 4 \mu \mathrm{K}$ rms for the $3.5^{\prime}$ pixelization), and to our statistical noise. Future experiments that seek to detect the much smaller B-mode polarization signal - particularly the lensing signal at small angular scales - will need to carefully account for the contribution to the B-mode signal by compact sources.

\subsection{Polarization of deep, high latitude maps at $145 \mathrm{GHz}$}

In Fig. 34 we present maps of the Stokes parameters $Q$ and $U$ at $145 \mathrm{GHz}$ in the deep survey region.

Unlike the I maps, here the CMB signal in each pixel is smaller than the noise. To estimate the signal to noise ratio $(\mathrm{S} / \mathrm{N})$ of the map, we have produced again two separate $145 \mathrm{GHz}$ maps, one from the first half (D1) of the observations in the deep region, and another from the second half (D2). In Fig. 35 we compare the histogram of the $Q$ (and $U$ ) data from the sum
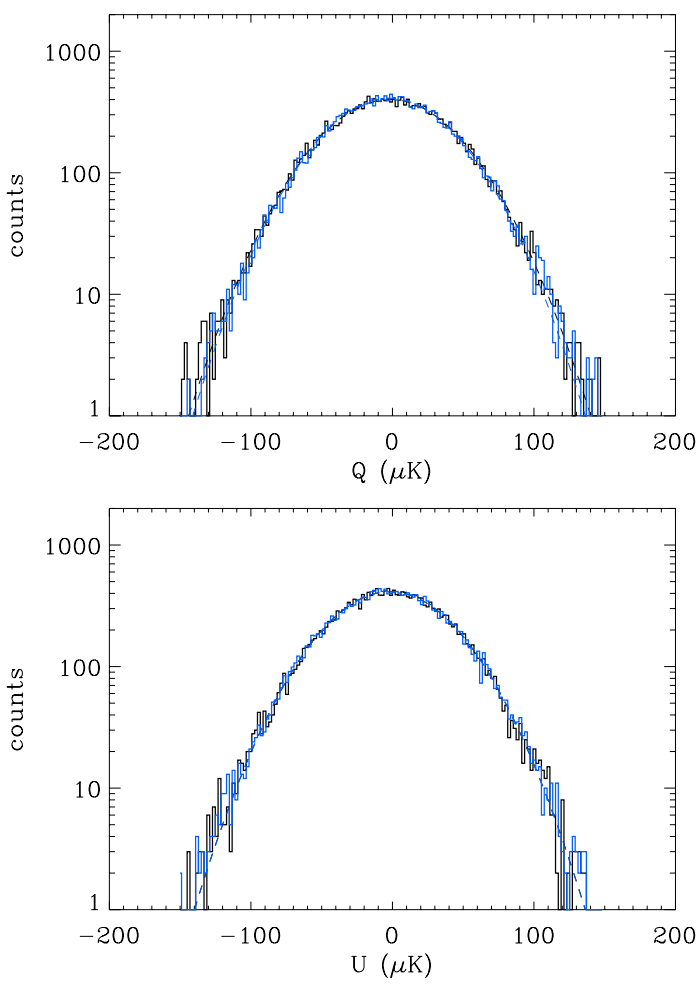

Fig. 35. Comparison of the $145 \mathrm{GHz}$ maps of Stokes $Q$ (top) and $U$ (bottom) taken in the two halves of the deep survey. The pixelization has NSIDE $=1024$ (3.5' pixels). We histogram pixel values resulting from the sum (heavy line) and difference (light line) maps. The sum and difference histograms are very similar: at the level visible here there is no indication of systematic effects that could arise from changes of observation conditions during the survey.

map (D1+D2)/2 to the same histogram from the difference map (D1-D2)/2. The difference histogram is very similar to the sum histogram, confirming that the signal is small with respect to the noise. For this reason we do not expect any CMB polarization structure to be visible "by eye" in the $Q$ and $U$ maps. The remarkable agreement of the sum and difference histograms in Fig. 35 indicates the stability of the system and the absence of systematic effects over the 6 days of measurement on the deep survey.

In Fig. 36 we plot the histogram of the measured polarization vector directions, computed as $\alpha_{i}=(1 / 2) \arctan \left[U_{i} / Q_{i}\right]$. Since the region observed by $\mathrm{B} 03$ is large compared to the typical correlation scale $\left(\sim 1^{\circ}\right)$ characteristic of $\mathrm{CMB}$ polarization, and since the two signals are dominated by uncorrelated noise, we naively expect a uniform distribution of the $\alpha_{i}$. The data plotted in Fig. 36 approximately confirm this expectation. The sinelike deviation present in the data is due to the anisotropy of the noise. Even though the 8 polarized detectors cover all possible directions (in steps of $\pi / 8$ ), the rotation of the focal plane with respect to the sky during the flight is small enough that for each direction of polarization only a few detectors have most of the statistical weight. If one of the bolometers is noisier than the others (see Table 6), the excursions of the noise will be larger in that direction, and the measured polarization will be preferentially oriented in the same direction. This has been confirmed by repeating the same analysis on maps obtained from realistic noise simulations. The $\mathrm{B} 03$ data are in full agreement with the distribution of the results of the simulations, which are plotted for comparison in Fig. 36. We can conclude that anisotropy effects are contained within $\$ 10 \%$ and are well described by our 


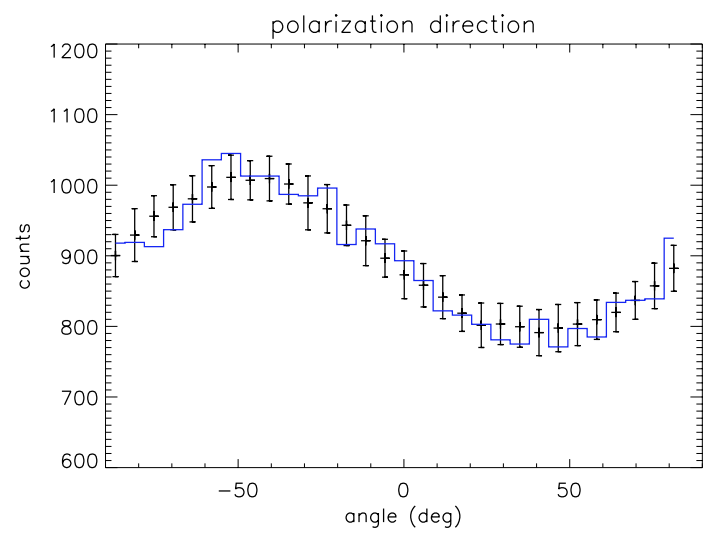

Fig. 36. Histogram of the directions of the polarization vectors, computed as $\alpha=0.5 \arctan [U / Q]$, in the deep survey at $145 \mathrm{GHz}$ (blue line). The $\sim 15 \%$ sinusoidal deviation from uniformity is due to unequalized noise of the different PSBs. This is confirmed by the analysis of maps obtained from realistic noise simulations (black data points with $1-\sigma$ error bars).

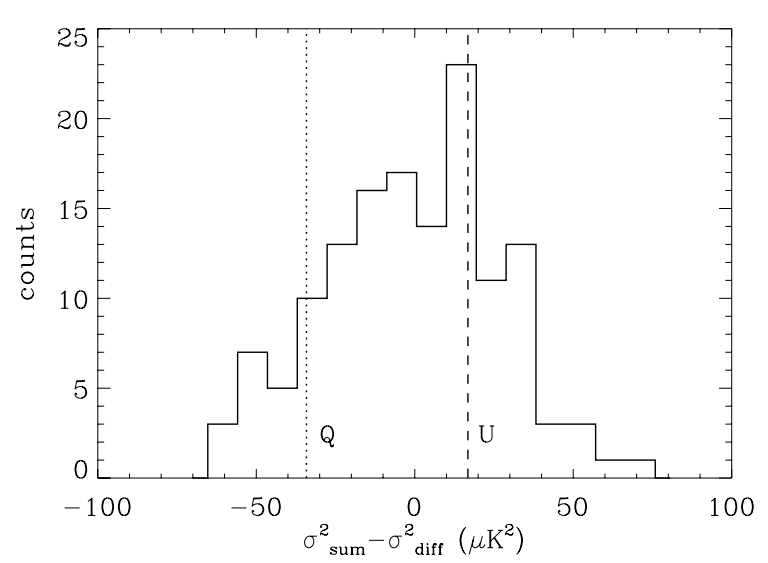

Fig. 37. Histogram of the quantity $\sigma_{O \text {,sum }}^{2}-\sigma_{O \text { diff }}^{2}$ from simulations of the B2K observations of the Deep Region. The simulations used the concordance model to generate $\mathrm{CMB}$ maps and realistic noise simulations for all the $145 \mathrm{GHz}$ detectors. The measured values of $\sigma_{Q \text {,sum }}^{2}-\sigma_{Q \text {,diff }}^{2}$ and $\sigma_{U \text {,sum }}^{2}-\sigma_{U \text {,diff }}^{2}$ are plotted as vertical dashed lines.

simulations. These effects can thus be treated in a self consistent way by the map making and by the spectral estimation analysis and simulation procedures.

From the sum and difference maps we compute the quantities $\sigma_{Q \text {,sky }}^{2}=\sigma_{Q \text {,sum }}^{2}-\sigma_{Q \text {,diff }}^{2}$ and $\sigma_{U \text {,sky }}^{2}=\sigma_{U \text {,sum }}^{2}-\sigma_{U \text {,diff }}^{2}$. In the absence of systematics, these are estimators of the mean square polarized signal from the sky. From a Gaussian fit to the $Q$ histograms we find $\sigma_{Q \text {,sum }}=(42.5 \pm 0.2) \mu \mathrm{K}$, while $\sigma_{Q \text {,diff }}=(42.9 \pm 0.2) \mu \mathrm{K}$. Analogously for $U$ we get $\sigma_{U \text {,sum }}=$ $(42.0 \pm 0.2) \mu \mathrm{K}$, while $\sigma_{U \text {,diff }}=(41.8 \pm 0.2) \mu \mathrm{K}$. These results do not allow one to extract the small sky signal (we expect $\sim 4 / \sqrt{2} \mu \mathrm{K}_{\mathrm{rms}}$ for $Q$ and $U$ in the concordance model with $3.5^{\prime}$ pixelization), but are fully consistent with the level of noise of our receivers (see Fig. 37).

In fact the uncertainty in $\sigma_{Q \text {,sky }}^{2}$ and $\sigma_{U \text {,sky }}^{2}$ obtained from realistic simulations of the instrumental noise and of the observations is $25 \mu \mathrm{K}^{2}$ (see Fig. 37). So we have $\sigma_{Q \text {,sky }}^{2}=(-34 \pm$ 25) $\mu \mathrm{K}^{2}$, and $\sigma_{U \text {,sky }}^{2}=(17 \pm 25) \mu \mathrm{K}^{2}$. For the polarization degree we have $P^{2}=\sigma_{Q \text {,sky }}^{2}+\sigma_{U \text {,sky }}^{2}=(-17 \pm 35) \mu \mathrm{K}^{2}$. This sets an upper limit to the rms linear polarization of the sky of $P_{\mathrm{rms}}<7.1 \mu \mathrm{K}$
(95\% confidence level). This limit is close to the $4 \mu \mathrm{K}_{\mathrm{rms}}$ expected for the $\mathrm{CMB}$ polarization signal in the $3.5^{\prime}$ pixelization.

Power spectrum methods provide much more powerful tools to extract the sky polarization from noisy maps, since it is much easier to separate different components according to their multipole content. In particular the effect of low-frequency noise and drift mostly affects the low multipoles $\ell \lesssim 50$. While the rms analysis above does not separate the different multipoles, the spectral analysis does; artifacts at low multipoles can be removed, allowing a much more sensitive probe for signal at higher multipoles. The application of these methods to B03 is described in detail in Piacentini et al. (2006) and Montroy et al. (2006), where it is shown that there is in fact a CMB polarization signal hidden in the $Q$ and $U$ maps described here.

\section{Conclusions}

The results of this flight of BOOMERanG demonstrate the effectiveness of balloon-borne $145 \mathrm{GHz}$ PSBs in the measurement of $\mathrm{CMB}$ anisotropy and polarization, and in the survey of mm-wave emission from the interstellar medium.

In our three frequency bands the long duration balloon platform offers an excellent tradeoff between cost and performance. With bolometers cooled to $0.3 \mathrm{~K}$, observing through an uncooled telescope, the stratosphere at altitudes $\gtrsim 25 \mathrm{Km}$ offers a very stable environment, and optimal loading conditions for these measurements.

During the entire flight, the B03 cryogenics and electronics produced the correct environment (in terms of operating temperature stability, radiative background level and stability, and electromagnetic disturbances) to operate the PSBs. These performed very closely to the theoretical limit, as described in Sects. 6.4 and 6.5. The attitude control system allowed us to reconstruct the pointing of the telescope with an accuracy of a few arcmin, sufficient for the main purpose of the experiment, i.e. the measurement of the power spectra of CMB anisotropy and polarization.

B03 produced maps of the sky at 145, 245 and $345 \mathrm{GHz}$ in two high-latitude regions and one region at low Galactic latitudes, in the Southern sky, producing a new survey of more than 1000 square degrees with $\sim 10^{\prime}$ resolution. At high latitudes degree and sub-degree scale anisotropy of the CMB is evident in the 145,245 and $345 \mathrm{GHz}$ maps. The rms fluctuation of the sky temperature measured from the $145 \mathrm{GHz}$ map is perfectly consistent with the current $\Lambda \mathrm{CDM}$ model for the anisotropy of the CMB. The $145 \mathrm{GHz}$ Stokes $Q$ and $U$ maps presented here allow a statistical (power spectrum) detection of $\mathrm{CMB}$ polarization at $145 \mathrm{GHz}$, as discussed in companion papers.

The approach discussed here also shows the effectiveness of multi-band observations in monitoring the foreground signal. The Galactic and extragalactic foreground is negligible $(\$ 10 \%$ compared to the cosmological signal both in anisotropy and in polarization) at $145 \mathrm{GHz}$ and at high Galactic latitudes. Based on the SFD maps, about $40 \%$ of the sky has ISD contamination equal or lower than in the deep survey region studied here. Future B-modes searches, seeking a much smaller polarization signal, will be heavily affected by the foregrounds measured here.

The results presented here are comparable in sensitivity to those published using interferometric techniques at lower frequencies; however, this measurement is the first using bolometric detectors, which can be scaled to large numbers and high sensitivity for future investigations of CMB polarization. In the nearer term, the measurements presented here provide a glimpse at what the Planck HFI instrument will achieve at $145 \mathrm{GHz}$; 
using the same technology (PSB detectors), the HFI will reach over the whole sky roughly the same sensitivity per pixel as was achieved by B03 in the deep survey.

Acknowledgements. We gratefully acknowledge support from the CIAR, CSA, and NSERC in Canada; Agenzia Spaziale Italiana, University La Sapienza and Programma Nazionale Ricerche in Antartide in Italy; PPARC and the Leverhulme Trust in the UK; and NASA (awards NAG5-9251 and NAG5-12723) and NSF (awards OPP-9980654 and OPP-0407592) in the USA. Additional support for detector development was provided by CIT and JPL. C.B.N. acknowledges support from a Sloan Foundation Fellowship, W.C.J. and T.E.M. were partially supported by NASA GSRP Fellowships. Field, logistical, and flight support were supplied by USAP and NSBF; data recovery was particularly appreciated. This research used resources at NERSC, supported by the DOE under Contract No. DE-AC03-76SF00098, and the MacKenzie cluster at CITA, funded by the Canada Foundation for Innovation. We also thank the CASPUR (Rome-ITALY) computational facilities and the Applied Cluster Computing Technologies Group at the Jet Propulsion Laboratory for computing time and technical support. Some of the results in this paper have been derived using the HEALPix package (Gorski et al. 1999).

\section{References}

Abroe, M. E., Borrill, J., Ferreira, P. G., et al. 2004, ApJ, 605, 607

Aliaga, A. M., Martinez-Gonzalez, E., Cayon, L., et al. 2003, New Astron. Rev., 47,821

Barkats, D., Bischoff, C., Farese, P., et al. 2005, ApJ, 619, L127

Battistelli, E. S., De Petris, M., Lamagna, L., et al. SPIE conference proceedings, Astronomical telescopes and instrumentation [arXiv: astro-ph/0209180]

Bennett, C., Halpern, M., Hinshaw, G., et al. 2003, ApJS, 148, 1

Benoit, A., Ade, P. A. R., Amblard, A., et al. , 2003, A\&A, 424, 571

Bernardi, G., Carretti, E., Cortiglioni, S., et al. 2003, ApJL, 594, 5

Bond, J. R., Jaffe, A. H., \& Knox, L. 1998, Phys. Rev., D57, 2117

Bond, J. R., Jaffe, A. H., \& Knox, L. 2000, AJ, 533, 19

Boyle, L. A., Steinhardt, P. J., \& Turok, N. 2004, Phys. Rev., D70, 023504

Bridle, S. L. , Zehavi, I., Dekel, A., et al. 2001, MNRAS, 321, 333

Brouw, W. N., \& Spoelstra, T. A. T. 1976, A\&AS, 26, 129

Bucher, M., Moodley, K., \& Turok, N. 2002, Phys. Rev., D66, 023528

Caderni, N., Fabbri, R., Melchiorri B., Melchiorri, F., \& Natale, V. 1978, Phys. Rev. D., 17, 1901; 1908

Carretti, E., Tascone, R., Cortiglioni, S., Monari, J., \& Orsini, M. 2001, New Astron., 6, 173

Carretti, E., McConnell, D., McClure-Griffiths, N. M., et al. 2005, MNRAS, 360, L10

Cartwright, J. K., Pearson, T. J., Readhead, A. C. S. , Shepherd, M. C., \& Sievers, J. L. 2005, ApJ, 623, 11

Catalano, A., Conversi, L., De Gregori, S., et al. 2004, New Astron., 10, 79

Copeland, E. J., Kolb, E. W., Liddle, A. R., \& Lidsey, J. E. 1993a, Phys. Rev. Lett., 71, 219

Copeland, E. J., Kolb, E. W., Liddle, A. R., \& Lidsey, J. E. 1993b, Phys. Rev. D, 48,2529

Cortiglioni, S., Bernardi, G., Carretti, E., et al. 2004, New Astron., 9, 297

Crill, B. P., Ade, P. A. R., Artusa, D. R., et al. 2003, ApJS, 148, 527

T. M., Dame, D., Hartmann, \& Thaddeus, P. 2001, ApJ, 547, 792

de Bernardis, P., De Gasperis, G., Masi, S., \& Vittorio, N. 1994, ApJ, 433, L1

de Bernardis, P., Ade, P. A. R., Bock, J. J., et al. 2000, Nature, 404, 955

de Bernardis, P., Ade, P., Bock, J., et al. 2002, ApJ, 564, 559

de Bernardis, P., Ade, P. A. R., Balbi, A., et al. 2003, in [arXiv:astro-ph/0311396], IAU Symp., 216, Maps of the Cosmos. Sydney 14-17 July 2003, ASP Conf. Ser.

de Gasperis, G., Balbi, A., Cabella, P., Natoli, P., \& Vittorio, N. 2005, A\&A, 436,1159

De Troia, G., Ade, P. A. R., Bock, J. J., et al. 2003, MNRAS, 343, 284

Delabrouille, J., Kaplan, J., \& The Planck HFI Consortium. 2002, in Astrophysical Polarized Backgrounds, ed. S. Cecchini, S. Cortiglioni, R. Sault, \& C. Sbarra (New York: AIP), AIP Conf. Proc., 609, 135

Djik, J., Van Diepenbeek, C. T. W., Maanders, E. J., \& Thurlings, L. F. G. 1974, I.E.E.E. Trans. on Antennas and Propagation, AP22, 513

Dodelson, S., \& Knox, L. 2000, Phys. Rev. Lett., 84, 3523

Doré, O., Teyssier, R., Bouchet, F. R., Vibert, D., et al. 2001, A\&A, 374, 358; see also http://ulysse.iap.fr/cmbsoft/mapcumba/

Douspis, M., Bartlett, J. G., Blanchard, A., \& Le Dour, M. 2001, A\&A, 368, 1

Dragone, M. 1974, IEEE Trans. AP, 22, 472; 1982, IEEE Trans. AP, 30, 331

Efstathiou, G., \& Bond, J. R. 1999, MNRAS, 304, 75

Farese, P., Dall'Oglio, G., Gundersen, J., et al. 2004, ApJ, 610, 625

Ferreira, P. G., \& Jaffe, A. H. 2000, MNRAS, 312, 89
Finkbeiner, D., Davis, M., \& Schlegel, D. 1999, ApJ, 524, 867

Fischer, M. L., Alsop, D. C., Cheng, E. S., et al. 1992, ApJ, 388, 242

Gordon, C., \& Lewis, A. 2003, Phys. Rev., D67, 123513

Gervasi, M., Boella, G., Cavaliere, F., et al. 2003, Proc. SPIE [arXiv: astro-ph/0301350]

Giommi, P., \& Colafrancesco, S. 2004, A\&A, 414, 7

Gorski, K. M., Hivon, E., \& Wandelt, B. D. 1999, in Proceedings of the MPA/ESO Cosmology Conference, Evolution of Large-Scale Structure, ed. A. J. Banday, R. S. Sheth, \& L. Da Costa, PrintPartners Ipskamp, NL, 37 (also [arXiv:astro-ph/9812350])

Gundersen, J. O., and the KUPID collaboration 2003, New Astron. Rev., 47, 1097

Guth, A., \& Pi, S. Y. 1982, Phys. Rev. Lett., 49, 1110

Hamilton, J. Ch, Benoit, A., the Archeops Collaboration 2003, Comptes Rendus Physique Académie des Sciences, 4, 853 [arXiv: astro-ph/0310788]

Hanany, S., Matsumura, T., Johnson, B., et al. 2003, IEEE Trans. Appl. Supercond., 13, 2128

Hedman, M. N., Barkats, D., Gundersen, J. O., McMahon, J. J., Staggs, S. T., et al. 2002, ApJ, 573, L73

Hoffman, Y., \& Ribak, E. 1991, ApJ, 380, L5

Holmes, W., Gildemeister, J. M., \& Richards, P. L. 1998, Appl. Phys. Lett., 72, 2250

Holzapfel, W. L., Wilbanks, T. M., Ade, P. A. R., et al. 1997, ApJ, 479, 17

Hu, W., \& White, M. 1997, New Astron., 2, 323

Jaffe, A. H., Ade, P. A. , Balbi, A., et al. 2001, Phys. Rev. Lett., 86, 3475

Johnson, B. R., Abroe, M. E., Ade, P., et al. 2003, New Astron. Rev., 47, 1067

Jones, R. J. 1953, J. Opt. Soc. Am, 43, 1

Jones, B. 2005, Ph.D. Thesis, California Institute of Technology

Jones, B., Bhatia, R. S., Bock, J. J., Lange, A. E. 2003, A Polarization Sensitive Bolometric Detector for Observations of the Cosmic Microwave Background 2003, SPIE, 4855, 227 [arXiv: astro-ph/0209132]

Jones, B., Ade, P. A. R., Bock, J. J., Bond, J. R., Borrill, J., et al. 2006a, ApJ, 647,823

Jones, B., Montroy, T., Crill, B., Contaldi, C., Kisner, et al. 2006b, A\&A, submitted [arXiv: astro-ph/0606606]

Kaiser, N. 1983, MNRAS, 202, 1169

Kalman, R. E. 1960, J. Basic Engineering, 82D, 35

Kamionkowski, M., Kosowsky, A., \& Stebbins, A. 1997, Phys. Rev. D, 55, 7368

Kaplinghat, M., Chu, M., Hainam, Z., Holder, G. P. L. Knox, et al. 2003, ApJ, 583,24

Keating, B. G., O’Dell, C. W., de Oliveira-Costa, A., Klawikowski, S., \& Stebor, N. 2001, ApJ, 560, L1

Keating, B. G., O’Dell, C. W., Gundersen, J. O., Piccirillo, L., Stebor, N., et al. 2003, ApJS, 144, 1

Keating, B. G., et al., http://www . astro.caltech.edu/ $\sim$ lgg/bicep/BICEP_color.pdf

Kogut, A., Spergel, D. N., Barnes, C., et al. 2003, ApJS, 148, 161

Kolb, E. W., \& Turner, M. S. 1990, The Early Universe (Addison-Welsey)

Komatsu, E., Kogut, A., Nolta, M., et al. 2003, ApJS, 148, 119

Kovac, J., Leitch, E., Pryke, C., et al. 2002, Nature, 420, 772

Kuo, C. L., Ade, P. A. R., Bock, J. J., et al. 2004, ApJ, 600, 32

Lange, A. E., Freund, M. M., Sato, S., et al. 1994, ApJ, 428, 384

Lange, A., Ade, P. A. R., Bock, J., et al. 2001, Phys. Rev. D, 63, 042001

Leach, S. M., \& Liddle, A. R. 2003, Phys. Rev. D, 68, 123508

Lee, C., Ade, P. A. R., \& Haynes, C. V. 1996, ESA SP-388, 81

Leitch, E., Kovac, J. M., Pryke, C., et al. 2002, Nature, 420, 763

Leitch, E., Kovac, J., Halverson, N., et al. 2005, ApJ, 624, 10

Lewis, A., \& Bridle, S. 2002, Phys. Rev. D, 66, 103511

Linde, A. 1983, Phys. Lett., B129, 177

Lubin, P., \& Smoot, G. 1981, ApJ, 245, 1

Mac Tavish C., Ade, P. A. R., Bock, J. J., et al. 2006, ApJ, 647, 799

Masi, S. 1984, Search for the Cosmic Background Polarization, in Gamow Cosmology, ed. R. Ruffini, \& F. Melchiorri, LXXXVI Course of the International School of Physics E. Fermi, Varenna, 1984, 310, Soc. It. Fisica, Bologna

Masi, S., Aquilini, E., Cardoni, P., et al. 1998, Cryogenics, 38, 319

Masi, S., Cardoni, P., de Bernardis, P., et al. 1999, Cryogenics, 39, 217

Masi, S., Ade, P. A. R., Bock, J. J., et al. 2001, ApJ, 553, L93

Masi, S., Ade, P. A. R., Bock, J. J., et al. 2002, in AIP Conf. Proc. 609, Astrophysical Polarized Backgrounds, ed. S. Cecchini, S. Cortiglioni, R. Sault, \& C. Sbarra (New York: AIP), 122

Mather, J. C. 1982, Appl. Opt., 21, 1125

Mauskopf, P. D., Bock, J. J., Del Castillo, H., Holzapfel, W. L., \& Lange, A. E. 1997, Appl. Opt., 36, 4

Montroy T. 2003, Ph.D. Thesis, University of California at Santa Barbara (http: //cmb.phys. cwru.edu/tom/thesis.ps)

Montroy, T., Ade, P. A. R., Bock, J. J., et al. 2006, ApJ, 647, 813 
Mukhanov, V. F., \& Chibisov, G. V. 1981, JETP Lett., 33, 532 (1981, Zh. Eksp. Teor. Fiz., 33, 549) see also [arXiv:astro-ph/0303077]

Nanos, G. P. 1979, ApJ, 232, 241

Natoli, P., de Gasperis, G., Gheller, C., \& Vittorio, N. 2001, A\&A, 372, 346

Natoli, P., Marinucci, D., Cabella, P., de Gasperis, G., \& Vittorio, N. 2002, A\&A, 383, 110

Netterfield, C. B., Devlim, M. J., Jarosik, N., Page, L., \& Wollack, E. J. 1997, ApJ, 474, 47

Netterfield, C., Ade, P., Bock, J., et al. 2002, ApJ, 571, 604

Padin, S., Shepherd, M. C., Cartwright, J. K., et al. 2002, PASP, 114, 83

Page, L., Hinshaw, G., Komatsu, E., et al. 2006, ApJ, submitted [arXiv: astro-ph/0603450]

Partridge, B., Nowakowski, J., \& Martin, H. M. 1988, Nature, 331, 146

Peiris, H. V., Komatsu, E., Verde, L., et al. 2003, ApJS, 148, 213

Piacentini, F., Ade, P. A. R., Bhatia, R. S., et al. 2002, ApJS, 138, 315

Piacentini F., Ade, P. A. R., Bock, J. J., et al. 2006, ApJ, 647, 833

Piccirillo, L., Ade, P. A. R., Bock, J. J., Bowden, M., Church, et al. 2002, in Astrophysical Polarized Backgrounds, ed. S. Cecchini, S. Cortiglioni, R. Sault, \& C. Sbarra (New York: AIP), AIP Conf. Proc., 609, 159

Ponthieu, N., Macías-Pérez, J. F., Tristram, M., Ade, P. A. R., Amblard, A., et al. 2005, A\&A, 444, 327

Polenta, G., Ade, P. A. R., Bock, J., Bond, J., Borrill, J., et al. 2002, ApJ, 572, L27

Polenta, G. 2004, Ph.D. Thesis, University of Rome La Sapienza

Prunet, S., Ade, P. A. R., Bock, J. J., Bond, J. R., Borrill, J., et al. 2001, proc. of the MPA/ESO/MPA conference, Mining the Sky [arXiv: astro-ph/0101073]

Richards, P. L. 1994, J. Appl. Phys., 76,

Readhead, A. C. S., Myers, S. T., Pearson, T. J., Sievers, J. L., Mason, B. S., et al. 2004, Science, 306, 836

Rees, M. 1968, ApJ, 53, L1
Romeo, G., de Bernardis, P., di Stefano, G., Masi, S., Piacentini, F., et al. 2002, in Experimental Cosmology at Millimetre Wavelengths, AIP Conf. Proc., 616,59

Ruhl, J. E., Ade, P., Bock, J., et al. , 2003, ApJ, 599, 786

Savage, R., Battye, R. A., Carreira, P., et al. 2004, MNRAS, 349, 973

Schlegel, D. J., Finkbeiner, D. P., \& Davis, M. 1999, ApJ, 500, 525

Seljak, U., Pen, U., \& Turok, N. 1997, Phys. Rev. Lett., 79, 1615

Song, Y. S., \& Knox, L. 2003, Phys. Rev., D68, 043518

Spergel, D. N., Verde, L., Peiris, H. V., et al. 2003, ApJS, 148, 175

Spergel, D. N., Bean, R., Dore', O., et al. 2006, ApJ, in press [arXiv:astro-ph/0603449]

Steinhardt, P. J., \& Turok, N. 2002, Science, 296, 1436

Subrahmanyan, R., Kesteven, M. J., Ekers, R. D., Sinclair, M., \& Silk, J. 2000, MNRAS, 315, 808

Tegmark, M., \& Zaldarriaga, M. 2000a, ApJ, 544, 30

Tegmark, M., \& Zaldarriaga, M. 2000b, Phys. Rev. Lett., 85, 2240

Tegmark, M., Strauss, M., Blanton, M., et al. 2004, Phys. Rev. D, 69, 103501

Tristram, M., Hamilton, J.-C., Macias-Perez, J. F., \& Renault, C. 2004, Phys. Rev. D, 69, 123008

Turner, M. S. 1993, Phys. Rev. D., 48, 5539

Villa, F., Mandolesi, N., Bersanelli, M., Butler, R. C., Burigana, C., et al. 2002, in Astrophysical Polarized Backgrounds, ed. S. Cecchini, S. Cortiglioni, R. Sault, \& C. Sbarra (New York: AIP), AIP Conf. Proc., 609, 144

Wollack, E. J., Devlin M. J., Jarosik, N., Netterfield, C. B., Page, L., et al. 1997, ApJ, 476, 440

Yamada, Y., Mitsuishi, A., \& Yoshinaga, H. 1962, J. Opt. Soc. Am., 52, 17

Yun, M., Beeman, J., Bhatia, R., Bock, J., Holmes, W., et al. 2003, Proc. SPIE, 4855,136

Zaldarriaga, M. 2003 [arXiv: astro-ph/0305272]

Zaldarriaga, M., \& Seljak, U. 1997, Phys. Rev. D, 55, 1830

Zaldarriaga, M., \& Seljak, U. 1998, Phys. Rev. D, 58, 023003 